United States Department of the Interior

Geological Survey

National Center for Earthquake Research

345 Middlefleld Road

Menlo Park, California 94025

\title{
USER'S MANUAL FOR MAPLOT
}

by

Robert Nowack

OPEN FILE REPORT 80-306

Th1s report is preliminary and has not been edited or reviewed for conformity with Geological Survey standards or momenclature

$$
\text { Pebruary, } 1980
$$




\section{Contents}

Section I.

Section II.

Section III.

Section IV.

Section V.

References

Appendix A

Appendix B

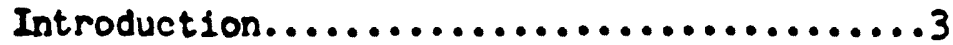

1. Brief Description...................

Getting started.......................4

1. The Multics Environment...............4

2. Interact1ve Input....................

3. Control Segments...................8

4. Output Devices....................11

Input data.......................... 15

1. Earthquake Data...................15

2. Station Data For Maps................19

3. Other Symbol Types For Maps...........20

4. Boundary Data For Maps..............20

Darthquake Sifting...................21

Particular Plots.....................23

1. Maps..........................23

2. Cross Sections....................29

3. Time/distance..................... 30

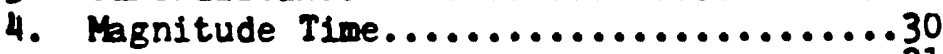

5. Magnitude Frequency.......................

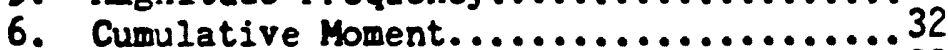

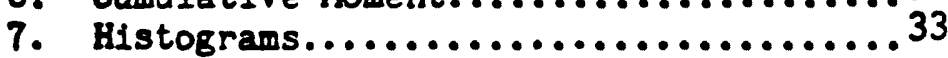

8. Frequency-Time................... 33

List of variables in control segments......34

Sample plots, interactive input and control

cezments...........................52 
I. Introduction

1. brief description

The purpose of the Maplot program is to construct plots of seismic location data. The program has been designed for use on the USGS Multics computer using the advanced graphics package Disspla.

Maplot has been written for the novice computer user. It has two different forms of input. There is a detailed interactive driver which prompts for the relevant input information. It can also be run with a formatted control segment.

There are a variety of ways to plot tabular seismic data. Maplot provides a number of ways but is not meant to be all inclusive.

Maplot has the capibility of plotting:

maps (earthquakes, stations, other symbols)

cross sections

time-distance plots

magnitude-frequency plots (semi-log)

cumulative moments plots

histograms of number or

summed moments with an axis (time distance depth)

time-frequency plots $(\log -1 \circ \mathrm{l})$

Maplot follows a very classical approach to plotting seismic data. A number of good examples to these sorts of plots can be found in (Utsu, 1961). 
For very simple interactive plotting, users with specific plots other than those included here should consider using Geolab which is inexpensive and easy to use.

An important feature of maplot, as a result of using the graphics package Disspla, is the device independence of the plots constructed. The user can output his plots on any of the output devices currently available with the USGS Multics computer. At Menlo Park, these Include:

Tektronix

Benson Lehner (pen plotter)

Houston Instruments (pen plotter)

Versatec

In addition, the user can direct his plot to the Disspla post processor which allows the user to output the same plot file to several different output devices.

The ability to interactively construct and preview plots allows for a wide variety of user Interaction and experimentation.

\section{Getting Started}

1. The multics environment

It is important for maplot users to have some familiarity with the multics computer. This includes login procedures, locating segments, and using one of the Multics text editors. This manual doesn't attempt to 
5

explain all the basic commands, although useful commands will be noted. A good reference to getting started on multics is the USGS Multics User's Manual. This can be obtained from the computer systems administrator.

Once the user is logged on, he must be able to locate various segments on public disk. A pathname is used to locate a particular segment. The entire storage space on Multics is organized into an inverted tree hierarchy. In pathnames, " $>$ " signs are used to separate different levels of the storage hierachy, for example in user disk space, the hierarchy would be, user disk directory (udd), project directories, person directories, and further sub-directories, etc. The pathname that locates the executable object segment of maplot is >udd > Caldata >RNowack > map_dir >maplot2. There are several convenient ways on multics for remembering segment locations. The first is to construct a link in your working directory such as Ik Judd $>$ Caldata $>$ RNowack $>$ map_dir $>$ maplot2 Your links are saved in a table for each directory and are only applicable within that directory. Thus, when Maplot2 is called, the links for your working directory will be searched for an entry which specifies the particular location for Maplot2. For a particular directory, the links may be listed using command "ls $-1 \mathrm{kn}$.

Another way to remember the location of a segment in public disk is with the abbreviation processor. This allows the user to construct abreviations for commands or complicated pathname locations. The abbrev processor is turned on by typing the command; "ab". An abbrev is saved in the processor with the command. ".ab maplot2 >udd > Caldata> RNowack $>$ map_dir $>$ maplot2.n 
With the abbrev processor turned on, the abbrev "maplot2" is expanded out to the full pathname anytime it is typed at command level at the beginning of a line. The advantage of using the abbrev processor is that it is not tied to the particular directory you may be in. These abbrevs are saved in a segment in your home directory called USER. profile, and can be listed using the command ". 1".

On Multics, to execute a program at command level, simply type the segment name. Once the appropriate link or abbreviation for the pathname of Maplot2 has been made, then by typing "maplot2n, the user starts to execute the program.

Maplot2 does require several other user created segments. These segments contain the earthquakes, stations, or other type of data the user wants to plot. When prompted by the interactive part of the program, the user should specify the pathname location for these data flles in public disk (abbreviations won't be expanded by the fortran program). These segments must contain only one type of data with no imbedded blank lines.

A program for selecting earthquake data called "select2n can be used to construct earthquake segments from master earthquake files. This is located at:

$$
\sum \text { udd }>\text { Caldata }>\text { RNowack }>\text { select2 }
$$




\section{Interactive Input}

Once the program has been started, the interactive section will prompt for various parameters. A flow chart for the interactive section is given in Figure 1. First, the input prompts the users for a control segment. If the user types no, it assumes the user wants to create a plot from input at the terminal. After typing in the plot parameters, the user has the option to write these parameters to a formatted control segment. To avoid typing in parameters a number of times, it is important to write input out to control segments. The control segments can be overwritten. Finally the user can either execute the plot, terminate the session, or go back and alter parameters. In executing the plot, the program calls the particular plotting subroutines. The user may wish to terminate the program if he is simply creating various control segments to be used later. If an input error has been made, he may wish to go back and alter parameters. The driver doesn't repeat all the questions the second time. It asks whether the user wants to change various parameters for each block of input. If the user doesn't want to change the parameters in a particular block of input, this block of input questions will be skipped. In this way, one can skip thru the driver to the block of parameters which needs modification. The input then drops down again to the (plot-terminate-alter) branch.

The user can specify a control segment at the start of Maplot session and then either alter these control parameters or branch down to the plotting device block. If the user does not want to alter control segment parameters, the input will ask for changes in the plotting device, then branch down to the 


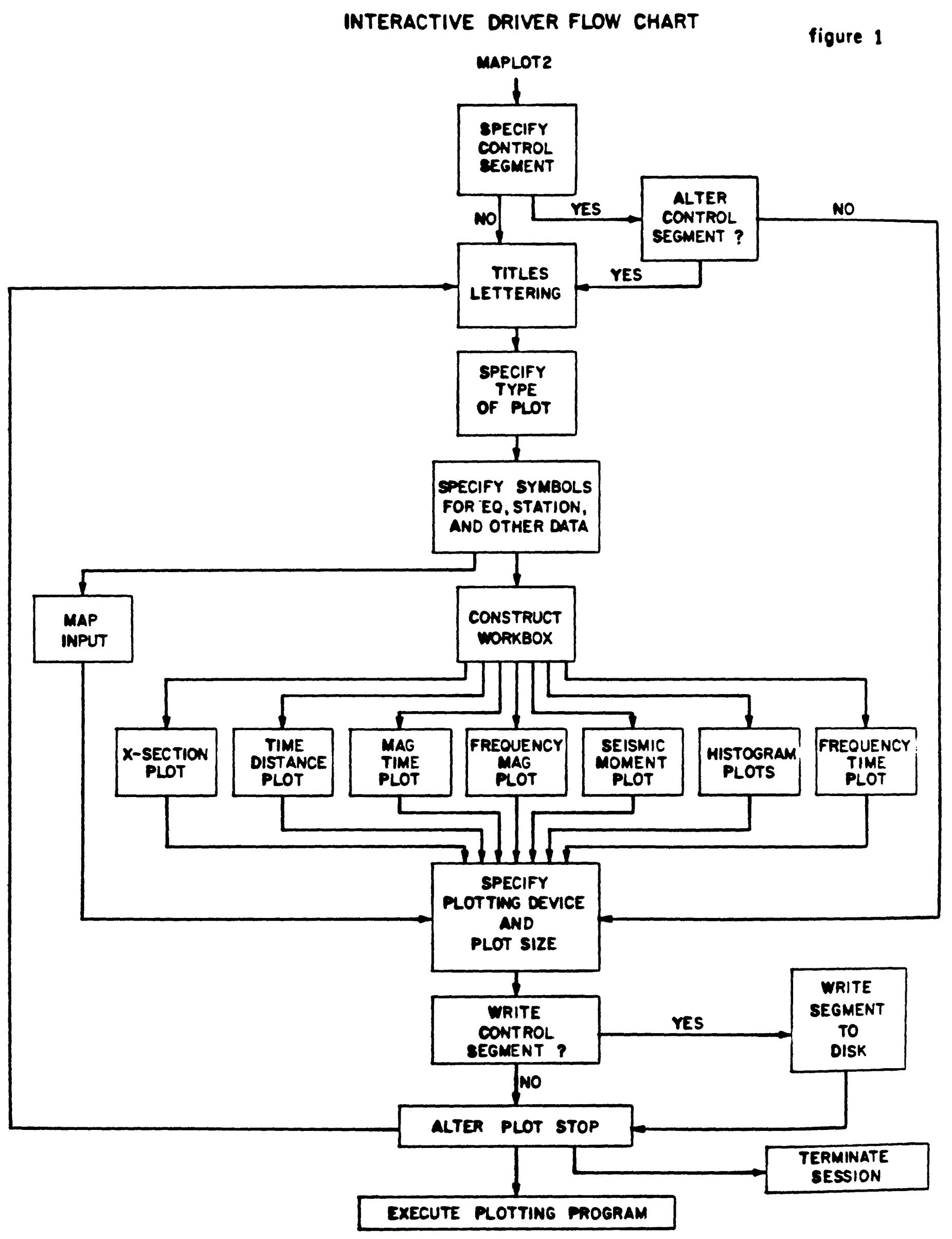


plot-terminate-alter point. If the user does want to interactively alter the control segment, the input will go down thru the block asking for any changes.

When using the interactive input, make sure to remember the decimal

points for real numbers. The program often gives examples of possible input. Follow the input type in these examples.

The interactive input is long so be patient when going through from the beginning. If you make an error and want to quit, hit break. This will stop execution. Then type "rl" to purge the job and type "maplot2" to start again. If you make an error while reading in data, type "new_proc" and start again.

\section{Control Segments}

Control segments can be written during an interactive session. This will allow the user to execute the program at some future time without answering all the interactive questions again.

The control segments can be modified in two ways. First, they can be modified by the interactive input. This is the same as altering parameters in the driver. The user can also modify the control segment with the text editor. Sample control segments are shown in appendix B.

Because these are read and written by formatted $I / 0$, the column spacing is important when making changes with the text editor. Any accidental changes of column fields when editing may cause a formatted read error.

In the control segments, the variable names are written along with the input numbers to help in text editing. A description of these different variables is given in appendix A. Because variables do interrelate with regard to making specific types of plots, it is important to compare your control segment with sample control segments for particular plots in appendix B. 
9

The sample control segments in appendix B can be found in public disk with the pathnames

$$
\text { \udd }>\text { Caldata }>\text { RNowack }>\text { map_dir }>\underset{\text { control. } 2}{\text { control. } 1}
$$

These are set up to create plots on a Tektronix terminal. When prompted for a control segment, give the pathname as above. A sample run using a test control segment would be:

maplot2

Welcome to Maplot

maplot info. read? no

do you want to specify an input control segment? yes

specify input control segment

\udd > Caldata > RNowack > map_dir > control.l

do you want to modify the parameters

in the control segment? no

do you want to change the output device or

plot size in inches? no

do you want these input parameters written to

an output control segment for future use? no

do you want to 1-plot, 2-terminate session,

or 3-alter input parameters again. 1 
There also exists and "ec" which allows the user to say the program name and the control segment name in one line.

First make an abbreviation for the "ec":

$$
. a b \text { maplot2a ec Judd > Caldata > RNowack > map_dir > maplot2a }
$$

Now type:

$$
\text { maplot2a > udd > Caldata > RNowack>map_dir > control.1 }
$$

This assumes that no changes are required to the control segment.

An equivalent batch job for a control segment set up to plot on an external plotter would look like:

ted

a

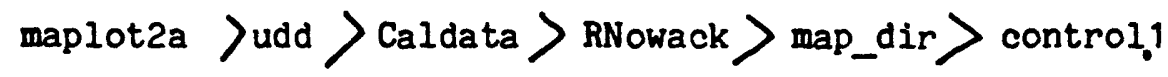

yes

w plop.absin

If

8.

This is an absentee segment and can be executed without the user being logged in at the terminal. This would be executed using the command

$$
\text { ear plop - rt }-q 1
$$

"ear" means enter absintee request.

"-rt" signifies that the computation should be restarted in case of a computer crash.

"q 1" signifies that the queue should be 1 which is daytime from 8:00 a.m. to 5:00 p.m. 
4. Output Devices

When the user specifies an output device other than tektronix, Maplot2 will write out a segment to disk. This segment contains the plot information in a form compatible with the external plotting device. The user mast then use an additional command which copies this plot segment to tape for plotting. For the tektronix, the plot information is sent directly to the screen and is not saved on disk.

In addition, Disspla has a post-processor which can be used to create device independent plot segments on disk. The post processor can be used to send the plot information to any of the plotting devices.

For Tektronix plotting, the vectors are plotted directly on the screen. The screen of the graphics terminal will be flashed several times to clear the screen for plotting.

Plots will not be plotted in absolute inches on the Tektronix screen. For plots less than $8-1 / 2$ by 11 inches, the plot is scaled to screen units. For plots larger than $8-1 / 2$ by 11 inches, the $y$ dimension of the plot will be scaled to just fit the screen. The $x$ axis of the plot may be truncated.

When previewing a plot, it is important to specify the plot size on the Tektronix to be the same as that for the external plotting device which plots in absolute inches. This is to ensure the proper proportions of the symbols, labels, and plot size for the final copy. Making the plot size on the Tektronix larger than $81 / 2$ by 11 , will have the unusual effect of changing titles and symbol sizes, but not the size of the plot on the screen. 
When using the Tektronix, you must specify the information rate for the graphics terminal. For direct ports to multics, the rate of the tektronix terminals is 9600 baud. This baud rate must be specified when prompted in maplot2.

For Versatec plotting, maplot2 writes a plot segment to disk named "vpltoo" in the user's working directory. After exiting Maplot2, this segment should be renamed to avoid overwriting it in subsequent runs of Maplot2. Use the command:

$$
\begin{array}{ll}
m & \text { vpltoo } \\
& 1=1,2 \ldots
\end{array}
$$

This plot segment must now be written to tape for plotting.

To generate a versatic plot, first link to;

$$
\text { lk } \quad>\text { iml }>\text { v_plot }>\text { gpt }
$$

Now, type "gpt" and the system will prompt for the necessary information. A nine digit USGS account number is needed for an external plot. A sample run of gpt which writes several plot segments to the same tape would be;

gpt

Enter tape volume name (for defult hit newline):

Enter account number: $\quad 100234509$

Do you wish to specify a wait interval in case no tape handlers are now available? yes

Enter walt time in minutes(-1 for unlimited): -1

Please enter file names to be placed in plot tape (separated by blanks): vplotl vplot2

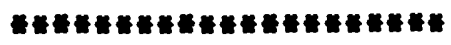


It is convenient to use one tape to plot several plot segments on versatec. Plots are limited to 43 inches in the $y$ direction on Versatec.

For Benson Lehner pen plots, a segment will be written to the users working directory with the same "bl_plot". After exiting Maplot2, rename it so it won't get overwritten the next time a Benson Lehner plot segment is generated. Use the command

$$
\begin{aligned}
\text { rn bl_plot } & \text { bplot } 1 \\
& 1=1,2 \ldots
\end{aligned}
$$

To generate a benson lehner plot, first

$$
\text { ik }>\text { iml }>\text { bl_lib }>\text { plotter.ec }
$$

The following command can then be used to plot a single plot segment.

$$
\text { ec plotter bplot1 } 30 \quad 1100089051
$$

where 30 is the paper size in inches $(10$ or 30$), 1$ is the pen size $(00-4)$, 100089051 is a valid USGS account number. Plots are limited to 30 inches in the $y$ direction on the Benson Lehner. This plotter is somewhat awkward for multiple plots because a separate tape must be used for each plot segment. For Houston pen plots, a file will be written to the user's working directory with the name "hous plot". Rename this segment after exiting the program to avold overwriting it.

$$
\begin{array}{r}
m \text { hous_plot hploti } \\
\qquad 1=1,2 \ldots
\end{array}
$$

To write these segments to tape for plotting, first:

$$
1 k\rangle \text { iml }>\text { houston }>\text { mult1_houston .ec }
$$


To make a plot, type:

ec multi_houston

This will prompt for the relevant information including a valid USGS account number and the plot segments to be plotted. Multiple plot segments can be written to the same tape for plotting. Houston plots have a maximum size of 36 inches in the $y$ direction. Because of a bug in the Houston software, a new_proc must be done between consecutive runs of Maplot which create Houston plots.

Pen plotters are much faster in computation time on Multics than the Versatec which must rasterize the entire plot area into tiny areas of Iight and dark. But once the plot segment is made, Versatec plots can be plotted in several minutes, while complicated pen plots may take several hours. There is a flat rate of $\$ 19$. per hour for plotting time on an external plotter regardless of the device (Oct 79). Thus, there is a trade off in expense between computation of rasterized plot segments for the Versatec on the computer and straight plotting time of complicated plots for the pen plotters. The Houston Instruments is a better bargain in speed and its ability to write multiple plot segments tapes than the Benson Lehner pen plotter.

Thus to choose between the Versatec and the Houston Instruments depends on the ratio of plot area and the number of vectors plotted since;

Versatec computer time $\propto$ plot area $\propto$ length2

pen plotter time $\propto$ number of rectors plotted $\propto$ plot time

Thus for large plots with only a few symbols drawn, it will be much more economical to use a pen plotter. But for a plot with a large number of vectors drawn, it will be more economical to use the Versatec. 
Finally, for slides and final graphs, it is important that plots be kept small (less than 12" square). Large plots, which aren't redrafted, don't seem to photograph well. Also, make all titles and labels fairly large.

\section{Section III Input Data}

Various forms of input data are allowed for maps. This includes earthquake data, station data, other types of symbols (for example, cities), and boundary or fault data. For plots other than maps, only earthquake symbols may be plotted. The data segments for these types of symbols must be prepared before the execution of Maplot2.

1. Earthquake Data

The formats allowed for earthquake data are:

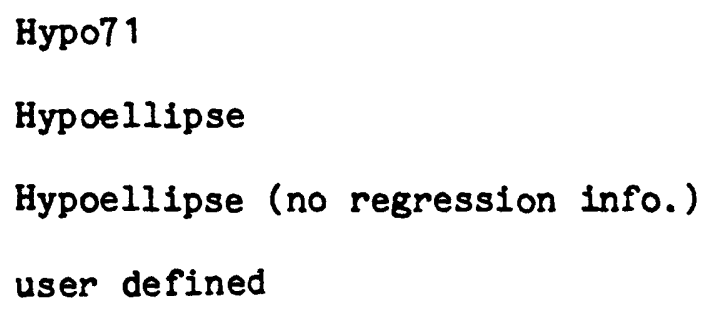

The variables on the read statement for the user defined are; kdate (AG), khrmn (A4), lat (integer degrees), "s" or blank (A1), xlat (real minutes), lon (integer degrees), "e" or blank (A1), xlon (real minutes), depth, xmag, quality (A1) 
By using the fortran "t" format, almost any earthquake format could be simulated. The Hypoellipse format has two magnitude spaces on the sumary card. The first is for amplitude magnitude, and the second is for duration magnitude. The user must specify one or the other depending on where the relevant magnitude information is punched on the card.

There is a limit of 10,000 earthquakes which can be read in by Maplot2. This could be redimensioned in the program if the need arose. If a segment has more events, only the first 10,000 will be read in for further processing. The earthquake segment should only have earthquake cards and no other types of data.

There are various kinds of symbols that can be used to plot earthquake data. These include:

1) A marker with no scaling. The allowed markers are shown in figure 2.

2) A marker with magnitude scaling. The markers allowed are shown in figure 2. A particular marker is scaled in size with increasing magnitude. The smallest magnitude symbol size must be given. Also, the magnitude increment and the increase in marker size in inches between consecutive magnitude increments must be given.

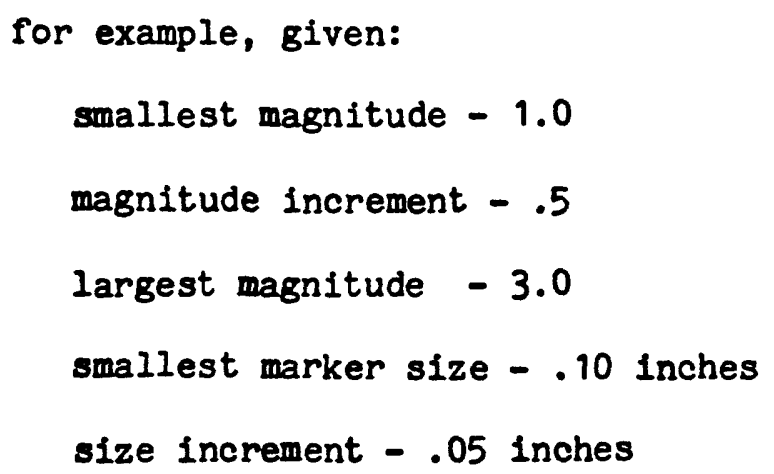


Then;

agnitude

$$
1.00-1.49
$$

$1.50-1.99$

$2.00-2.49$

$2.50-2.99$

marker size

in inches

.1

.15

.2

.25

Note that the smallest magnitude, 1.0, will be plotted, but the largest magnitude, 3.0, w111 not.

Also, an integral number of magnitude increments should fit in the total magnitude range.

3) A different marker with depth scaled with magnitude

There is a shallowest and deepest depth used to sift earthquakes in depth. The user must specify a depth increment starting from the shallowest allowed depth. Different markers will be used for progressively greater depth increments down to the maximum depth cutoff. The markers always start with marker 0 in Pigure 2 and increase. There should be less than 15 depth increments in the depth range to allow for a distinct symbol 0-14 in Plgure 2 .

For example, Biven;

shallowest depth - 20. km

greatest depth - 40. $\mathrm{km}$

depth increment - 5. $\mathrm{km}$

figure 2

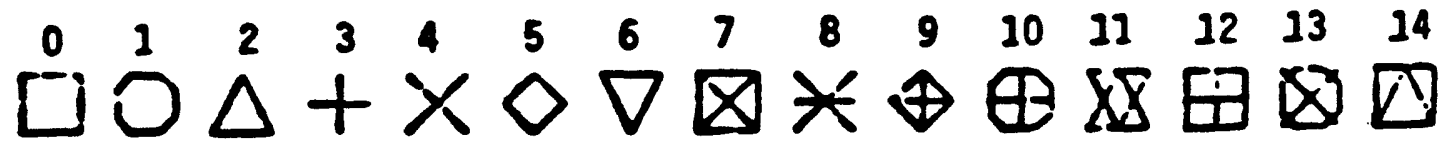


then;

event depth

$$
20.00-24.99
$$

25. -29.99

30. -34.99

35. -39.99

marker type

in figure 2

0

1

2

3

Note that shallowest depth, 20., is plotted but the greatest depth, 40 . $\mathrm{km}$, is not plotted.

These markers are scaled with magnitudes as described in symbol type 2 . 4) A number representing magntidue - no scaling. This symbol simply converts the magnitude to an integer by truncating and plots this integer. No magnitude scaling is done.

5) A number or letter representing depth down to $35 . \mathrm{km}$ - scaled with magnitude.

This symbol converts the depth to an integer by truncating. The symbol plotted then are related to depth by:

symbol plotted $\quad 0,1, \ldots, \quad 9, a, b, \ldots, y, z$,

depth in $\mathrm{km} \quad 0,1, \ldots, 9,10,11 \ldots, 34,35$, greater than $35 \mathrm{~km}$

These symbols are then scaled with magntitude.

6) A letter representing quality - scaled with magnitude. A quality of solution letter $A-D$ is plotted. These letters are then scaled with magnitude. 
2) Station Data

Station data can only be plotted on maps. The formats allowed by Maplot2 are:
1) Hypo71 (delay model)
2) Hypo71 (variable first layer)
3) Hypoellise
4) user defined

The variables in the user defined input list are;

lstat (A4), lat (integer degrees), "s" or blank, xlat (real minutes), lon (integer degrees), "en blank, xlon (real minutes)

Using the fortran "t" format will allow the user to construct a format appropriate to his data.

At present, there is a limit of 900 stations which can be read in. If there are more stations than 900 in the input segment, they will be ignored. Other sorts of data in the station segment will cause the program to bomb out. There are two types of symbols allowed for stations. The first is just a marker as in figure 2. The second type of symbol uses a marker with a four letter station name to the upper right of the marker. Both the marker size and the label size can be varied independently. 
3) Other Data for maps

Another type of symbol can also be plotted on maps. This may be cities, ranches, or other sorts of data. The only format for this is user defined. The variables in the read statement are

loth(A20), lat(integer degrees), "s" or blank for north(A1),

$x$ lat(real minutes), lon(interger degrees), "en or blank for west(A1), xlon(real minutes)

A maximum of 500 cards can be read in.

4) Boundary or fault data

There are various boundary files which can be used with Maplot2. These include the Disspla boundary files and user defined boundary segments.

The Disspla files don't require any format or input segment from the user. These Disspla files include;

1. mapdta .5 degree resolution

2. hershey

3. africa

4. antarctic

5. asia

6. australia

7. europe

8. north america

9. south america
10. pafrica

11. pasia

12. paustralia $.1-.5$ degree

13. peurope resolution

14. pnorth america

15. psouth america

16. coastlines - combined coastlines

17. political - combined political

19. usa med resolution

20. usa high resolution 
For user defined files, a particular form for the data is required. First, a card with blanks in the first three columns signifies that the pen should be lifted and the remainder of that card is ignored.

The variables in the read statement are (test (A3), tl, idline (alat (decimal degrees), alon (decimal degrees))). The "test" variable tests to see if the line is blank. The "t1" tabs back to column 1. The line then has "idline" pairs of latitude and longitude in decimal degrees. A sample format would be:

$$
(A 3, t 1,6(f 5.2, f 6.2))
$$

This is the format for the fault map of central California digitized by Rick Lester. This is located in:

$$
>\text { udd }>\text { caldata }>\text { RNowack }>\text { map_dir }>\text { calif2 }
$$

\section{Section III Earthquake Sifting}

Maplot2 has the capability to do simple earthquake sifting. For all plots, the user can plot earthquakes in a depth range and a magnitude range. The smallest and largest magnitude and the shallowest and greatest depth allowed must be specified. The largest magnitude limit and the greatest depth are not included in the interval. That is

$$
\begin{aligned}
& \text { xmag1 } \leqslant \text { magnitude }<\text { xmag2 } \\
& \text { xdep1 } \leqslant \text { depth }<\text { xdep2 }^{2}
\end{aligned}
$$

For maps, this is the only earthquake sifting done. Also for maps, no earthquake outside of the map area will be plotted. 
22

For all other types of plots, further sifting is done in addition to magnitude and depth. For all other plots, a work box is constructed as in Pigure 3.

The points $A$ and $A^{\prime}$ are specifled in latitude and longitude. $T h_{e}$ variables xwid1 and xwid2 are in $\mathrm{km}$ and specify the maximum distances away from the line A - A' to accept earthquakes. The variables $x d e p 1$ and $x d e p 2$ are the depth ranges as before. For all plots other than maps, earthquakes must be in this workbox and be in the proper magnitude range to be plotted.

For all plots other than maps, earthquake latitude and longitude are converted to distance in $\mathrm{km}$ and azimuth clockwise from north from the point $A$. This conversion is shown in figure 4.

The subroutine DELAZ is used to convert latitude and longitude on a sphere corrected for ellipticity to distance and azimuth. These values are then converted to $x^{\prime}$ and $y^{\prime}$ in $k m$ along $A-A^{\prime}$ and perpendicular to $A-A^{\prime}$. The values $\left(x^{\prime}, y^{\prime}\right)$ for each earthquake used must lie in the range:

$$
\begin{aligned}
& 0 . \mathrm{km} \leqslant x^{\prime} \leqslant\left(D I S T A N C E \text { fROM } A \text { to } A^{\prime}\right) \\
& x \omega / D 1 \leqslant y^{\prime}<x \omega / 02
\end{aligned}
$$

For all time plots, a beginning and ending time for the time axis must be specified. For time plots, earthquakes must have times within the ranges to be plotted. For other plots, no time sifting is done. 
The beginning and ending times must be specified as valid dates.

Thus

$740101 \quad 1845$ is correct while $740400 \quad 1845$ is incorrect.

The time axis can be specified in the following time units; 1 - hours, 2 - days, 3 - month (approx), 4 - years (approx). The subroutine CALJUL is used to convert the time differences between the axis beginning time and the earthquake times into real numbers in the time units specified. For example, the time difference between

73010100007401010000

in years would 1.00 , in months would be 12.00 , and in days would be 365.00 .

Section V Specific Plots

The following section give specific information on particular plots.

1) Maps

Earthquakes, station data, other symbols, boundary data, or any combination of these can be plotted on maps. Symbols lying outside of the map area will not be plotted. In addition, earthquakes must be in specific magnitude and depth ranges.

The map area is specified by YORIG and YMAX which are the lower and upper latitide and XORIG and XMAX which are the left most and right most longitude. It is important to keep in mind that west longitude and south latitude are negative.

In the interactive input section, all map coordinates should be given in integer degrees and real minutes. These are then converted to decimal degrees by the program. The control segments also use decimal degrees. 
For example:

Interactive input IORIG $-122 \quad 15.00$

Program computation and control segents -122.25

For maps, scaling is done by specifying the length in inches of the central meridian between the lower and upper latitude limits of the ap. The map is then adjusted so that the latitude range of the central aeridian just fits in the number of inches specified. The length in Inches of the $x$-axis has no effect on map scaling. It is important to allow enough space on the $x$-axis to avoid truncating the longitude range on either side of the map. The length required on the $x$-axis in relation to the y-axis length is dependent on the map projection. As a preliminary rule, always specify more inches on the $x$-axis than that on the $y$-axis.

At present, there is no specific scaling factor in the mapping section of DISSPLA which determines map scale. This deficiency may soon be changed by DISSPLA themselves or by using our own projections. Another source of problems is the interaction between the aultics environment and DISSPLA map projections. DISSPLA projections are only single precision which may result in loss of significant digits. Also, Multics has a restricted exponent range between 10-38 - 10+38. Inis my cause unforeseen exponent overflow or underflow conditions when using certain latitude ranges wth specific projections. In addition, certain coarsely sampled user boundary flles may cause overflow conditions near - dges of certain ap projections. Specific projections will be discussed below.

There are a wide variety of DISSPL map projections. These all have particular latitude and longltude ranges as well as apecific types or input. 
Several definitions first may be appropriate. First, when a map preserves shapes of small parts of mapped surfaces, even though it can't preserve shape over a large area, such as an entire continent, it is considered CONFORMAL. If the relative area of all features of a globe is maintained during projection, the projection is said to be EQUAL AREA. A flat map of a globe can't be both equal area and conformal.

Although no map projection offers uniform scale, some have uniform scale in preferred directions.

\section{A) Cylindrical Projections}

1) Cylindrical and Exact Cylindrical. The Cylindrical projection doesn't transform the data, it simply displays the coordinates as they are. The Exact Cylindrical corrects for the earth's ellipticity. There is one standard parallel at the equator. These projections are neither conformal or equal area. The latitude range is $-180 \mathrm{~s}$ to $+180 \mathrm{~N}$ but must not span more than 180. $x$ and $y$ are scaled independently.

2) Mercator and Exact Mercator. The Mercator projection is a cylindrical projection with the line of tangency with the globe being the equator and the axis of the cylinder coinciding with the globes N-S pole. This is a conformal projection which preserves local shape but not area. Parallels and meridians are straight lines at right angles. The Exact Mercator corrects for the earth's ellipticity. Lines of constant bearing plot as straight lines but great circles don't. 
The latitude range is

$$
\text { -85. } \leqslant \text { lat } \leqslant 85 .
$$

The map may appear anywhere in the longitude -540 . to +540 ., but the total map span must be less than 7200 or 2 cycles.

3. The Transverse mercator

The Transverse Mercator is projected onto a cylinder widh the axis on the equator and the line of tangency being a line of longitude. This line of longitude, which is picked as the center longitude of the map, has no distortion.

4. The Universal Transverse Mercator

The UTM projection was developed by the U.S. Army as a world wide grid system. The world is divided into $\mathrm{N}-\mathrm{S}$ zones of longitude with each zone being 6 degrees wide and having its own central meridian. In California, the zones go from -126 to -120 degrees and -120 degrees to -114.00 degrees. The central meridians are -123 and -117 degrees.

B. Conic Projections

Conic projections involve projecting onto imaginary cones fit over the globe. All Disspla conic projections have two standard reference parallels. The conics supplied by Disspla have a cone axis coincident with the north or south pole.

1) Bi-parallel Conic Conformal (Lambert). The meridians are straight line which intersect when extrapolated to either the north or south pole, depending on the hemisphere of the map. True shapes are depicted at local scales. The parallels are spaced at increasing intervals the farther north or south from the reference parallel. See below for ranges. 
2) Bi-parallel Equal Area conic (albers). The parallels are spaced at decreasing intervals the farther north or south of the standard parallels so as to preserve area. see below for ranges. 3) Polyconic on an infinitesimal-graticule. This is an extension of a simple conic using many tangent cones instead of just one. The polyconic is conformal and also adjacent maps can be matched with no discrepency. This is used extensively for topographic maps.

\section{Notes for conic projections}

a) The reference parallels are assumed to be at 1/4 the latitude span from the top and bottom of the map unless otherwise specified. b) The latitude limits must not span the equator. Thus the latitude limits must lie in the range. $-90-0$ or $0.0-900$. c) The longitude must not span more than 3600 .

C) Azimuthal Projections

All azimuthal projections are based on the concept of the globe tangent to a plane. The map pole is defined to be the point at which the globe touches the plane of projection. The map pole is at 00 latitude and 00 longitude unless otherwise given. The characteristic of azimuthal projections is that directions to all points from the map pole are not distorted during projection.

1) Gnomonic. This projection is neither conformal or equal area. Also, the map distorts badly at the corners. It does have the important feature that great circles on the globe project as straight lines on the map. The latitude and longitude limits must not exceed 850 from the map pole. 
2) Orthographic Projection. This is a true projective view of the globe as seen from infinity. It is neither conformal or equal area. See below for ranges.

3) Stereographic. This is a conformal projection sometimes used for large area maps of the entire hemisphere. See below for ranges.

4) Azimuthal Equidistant. This projection is neither conformal or equal area. It has the feature that lengths from the map pole on the map correspond to lengths on the surface of the sphere. See below for ranges.

5) Azimuthal Equal Area (Lambert). This is an equal area projection. It preserves the area of concentric rings about the map pole. See below for ranges.

Except for the Gnomonic, the azimuthal projections can have latitude and longitude limits up to 900 around the map pole. Even larger limits may be set with the warning that the map may fold back on itself.

D) Elliptical Projections

These projections are sometimes desirable for maps of the entire globe. Several of these projections have important area preserving properties.

1) Molleweide. This is an equal area projection with lines of constant latitude being parallel. This projection distorts at the poles. 
2) A1toff's (Hammer's) projection. This is also equal area, but lines of latitude are not parallel.

3) Sanson (Flamsteed) projection. This projection has lines of constant latitude straight and equally spaced. It has a somewhat unusual shape and is distorted at the edges and poles.

4) Simple Elliptical. This is not truly equal area but somewhat resembles the Molleweide projection. It has a very simple projecting equation.

For the elliptical projections, the longitude may span up to 3600 in the range -3600 to +3600 . The longitude must be in the range -900 to +900 .

2) Cross Sections

The cross sections are defined by the map coordinates $A$ and $A^{\prime}$, the maximum distance away from $A-A^{\prime}$ in $\mathrm{km}$, and the depth ranges. Only earthquakes in the workbox will be plotted. The $x$ axis goes from point $A$ (0. $\mathrm{km})$ to point $A^{\prime}(x \times \mathrm{km})$. The distance $x x$ is calculated from the coordinates of $A$ and $A^{\prime}$. The user must specify the $x$-axis increment. The $x$-axis will draw out an integral number of increments from point $A$. Thus the $x$-axis will terminate an integral number of increments with a length less than or equal to the total axis length of $x x \mathrm{~km}$. Earthquakes will still be plotted out to $x x$.

The depth range is given by $x d e p 1$ and $x d e p 2$. The user must also specify the $y$-axis increment. The y-axis will be drawn out in an integral number of increments. 
As described in the earthquake sifting section, the coordinates of the earthquakes have been transformed into workbox units along $A-A^{\prime}\left(x^{\prime}\right)$, perpendicular to $A-A^{\prime}\left(y^{\prime}\right)$, and depth $(z)$. For cross sections along $A-A^{\prime}$, the coordinates $\left(x^{\prime}, z\right)$ are plotted. For perpendicular views of $A-A^{\prime}$, the coordinates $\left(y^{\prime}, z\right)$ are plotted.

\section{3) Time Distance Plot}

In addition to workbox sifting, earthquakes must lie in the prescribed time range.

The $y$-axis is the distance from point $A$ in $\mathrm{km}$ in transformed workbox coordinates $\left(x^{\prime}\right)$. The user must enter the distance increment in $\mathrm{km}$. Time is plotted along the $\mathrm{x}$-axis starting at the specified beginning time. The time can be plotted in hours, days, months (approx), or years (approx). The beginning and ending times are given in the form; 6901011605790312340

where the first 6 digits are (yr, month, day) and the last four digits are (hour, min). The earthquake times are then transformed into a time from the beginning time in the specified time units.

4) Magnitude-time plots

This is a very simple plot which plots spikes with time. The length of the spike is proportional to the magnitude of the event. The time is in user defined units of hours, days, months (approx), or years (approx). Earthquakes must be located in the workbox to be plotted. 


\section{5) Magnitude Frequency}

This will plot cumulative or incremental (density) numbers of earthquakes with magnitude on a semi-log plot. The summing increment must be specified on the magnitude axis.

A symbol is drawn in the center of each summing interval. For the density plot, each symbol represents the number of events in that interval. For the cumulative number plot, each symbol represents the cumulative number of events with magnitudes greater or equal to the summing increment. In both plots, a line is drawn connecting the symbols.

For the cumulative number plots, the resulting b-value for the event distribution can be calculated. "b" is the slope in the Gutenberg-Richter formula for cumulative number of events with magnitude: $\log N$ (cumulative) $=a+b \operatorname{Mag}$

A smallest magnitude must be specified for calculation of $b$.

The maximum likelihood estimate of "b" as discussed by Aki (1965) is given by:

$$
\hat{b}=\frac{\log _{10} e}{\bar{M}-M_{\min }}
$$

where $M$ is the average magnitude of the earthquake population and $M_{\min }$ is the minimum magnitude. Confidence intervals for this estimate can be found in Aki (1965). The values for $b$ and $M_{\text {MIN }}$ are written at the top of the cumulative frequency plot. 
For these semi-log plots, a minimum number ( $y$-value) and the number of cycles on the $y$-axis must be given. The user must also specify the magnitude increment for labeling the $x$-axis. Xmag 1 and $X m a g 2$ are used as the smallest and largest magnitude on the $x$-axis. Xmag 1 and the value $M_{\text {min }}$ used in the value computation must be specified separately. 6) Cumulative moment plot

In addition to workbox sifting, the events must lie in the specified time range. The earthquake times are converted into times from the beginning time in the user specified time units.

The seismic moment is a source dependent parameter whereas magnitude depends on the source, instrument and propagation path. For a particular region and instrument, approximate relations between magnitude and moment can be empirically constructed. These relations have the form:

$$
M_{0}=10(a+b \text { Mag })
$$

where $M_{D}$ is the seismic moment in dyne-cm and $a, b$ are constants. For example, in southern California, Thatcher and Hanks (1973) found the following relations:

$$
M_{0}=10(16+1.5 \mathrm{mag})
$$

With this relation the moment corresponding to particular magnitudes would be:

$\begin{array}{cc}\text { Magnitude } & \text { Seismic Moment (dyne-cm) } \\ 1 & 3.16 \times 1017 \\ 2 & 1.0 \times 1019 \\ 3 & 3.16 \times 1020 \\ 4 & 1.0 \times 10^{222} \\ 5 & 3.16 \times 1023 \\ 6 & 1.0 \times 1025\end{array}$


The program requires the user to specify values of $n_{a} n$ and ${ }^{n}{ }^{n}$ relevant to the region where his events are located.

The resultant seismic moments are then sumed with time to obtain a cumulative plot. The $y$-axis is self scaling to the data. The events must be chronological in time in the earthquake data segment.

7) Histogram plots

This is a whole series of different plots which represent the data as histograms. The number of events or the summed moment can be plotted with time, distance along $A-A^{\prime}$, distance perpendicular to $A-A^{\prime}$, or depth. The summing increment for the appropriate $x$-axis units must be specified. The y-axis is linear and portrays either numbers or sumed moment. For simplicity, the $y$-axis is self scaling to the data.

8) Frequency time-plot

This is a $\log -\log$ plot of the frequency of events in time increments after the beginning time. For aftershock sequences, the number of events in a unit time interval at a time from the main shock follow an emperical relation;

$$
n(t)=\frac{A}{(t+C)^{p}}
$$

where $A, C$ and $P$ are constants for the sequence. For many sequences, $P$ is found to be slightly greater than 1 (Utsu, 1961). This formula is known as Omori's law.

On a log-log plot, a frequency time plot of an aftershock sequence with $P=1.00$ would have a slope of -1 . In order to have equally spaced points in time on a $\log$ scale, the suming window increases with time. The number of events is then divided by the time length of the summing window. The suming window is specified by the user in fractions of a log-cycle. For an increment equal to one cycle, a symbol is plotted in the geometric center of each log-cycle. 


\section{Appendix A}

This appendix gives an explanation of the variables in the control segment. The control segment has 30 lines of input parameters. The variable names are listed to the left of each variable number. This segment is read and written with formatted I/O statements and thus the columns are important.

The variables and formats for each line of the control segment will be given

line 1 - format $(7 x, 11,7 x, 14,7 x, 11,8 x, 11,6 x, 11,5 x, 11,5 x, 11)$.

$$
\begin{aligned}
& \text { device - output plotting device } \\
& 1 \text { - Tektronix } \\
& 2 \text { - Versatec } \\
& 3 \text { - Benson Lehner } \\
& 4 \text { - Calcomp (Reston) } \\
& 5 \text { - Disspla post processor } \\
& 6 \text { - Houston plotter }
\end{aligned}
$$

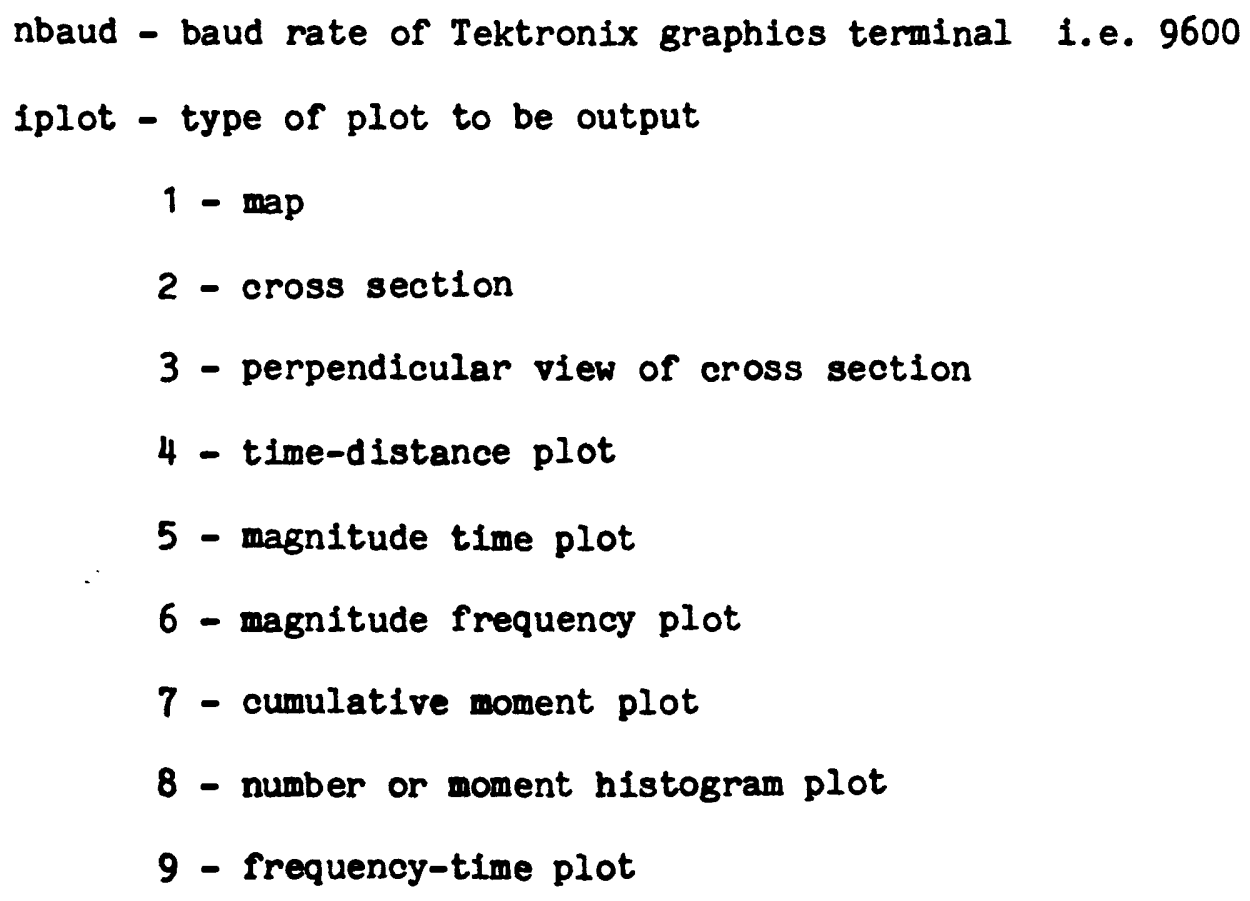




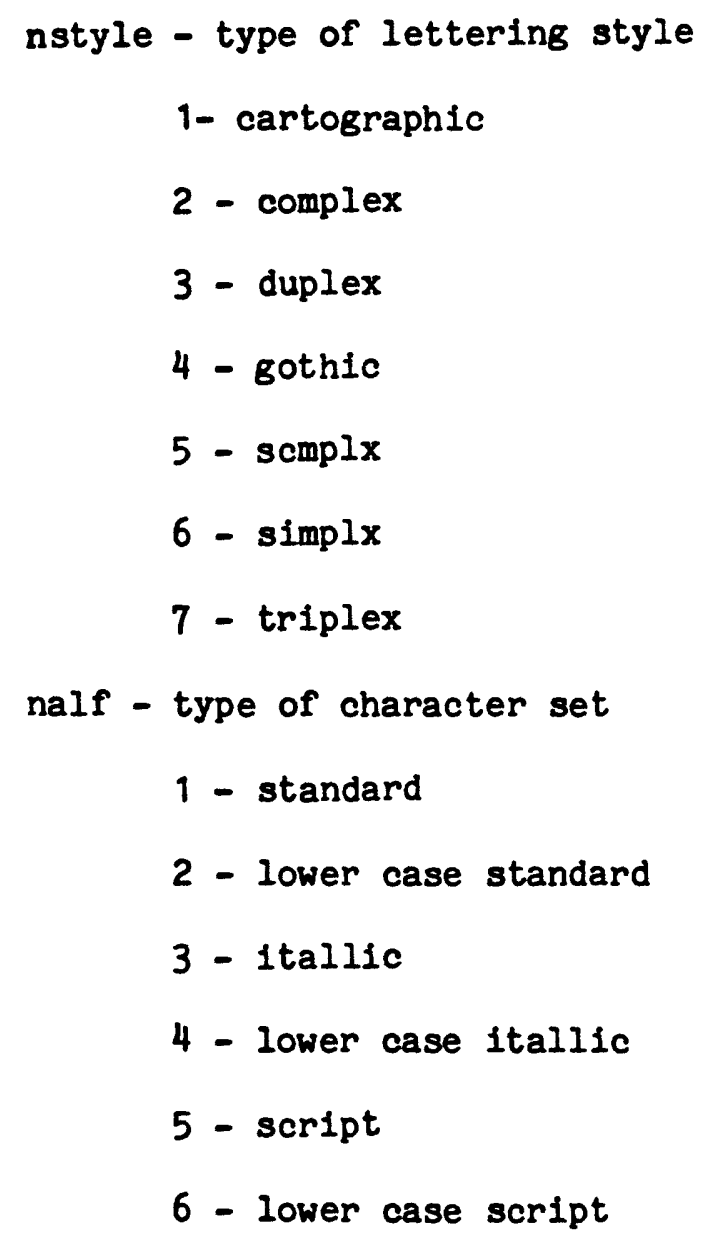

Iine 2 format $(3 x, 11,4 x, 11,4 x, 11,7 x, 11,7 x, 11,7 x, 11,8 x, 11)$ ne - control for coding in earthquakes

0 - no earthquake data read in

1 - eq data will be read in

ns - control for reading in station data

0 - no station data reading

1 - station data will be reading

no - control for reading in other data

0 - no other data read in

1 - read in other type of data 


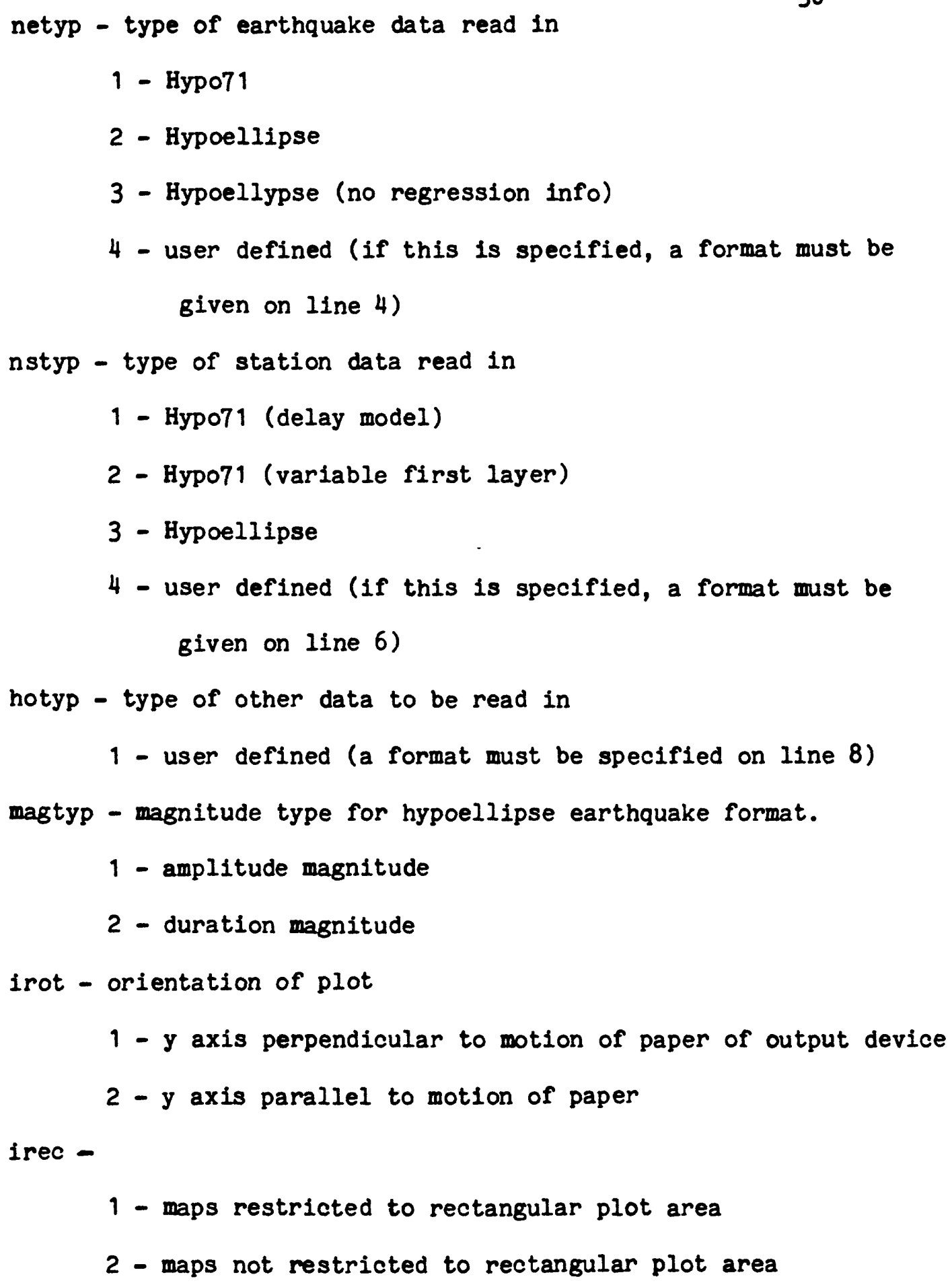


line 3 format $(11 x, a 60)$

equake seg - This field is used to specify the segment containing the earthquake data.

line 4 format $(A 80)$

This line is used to specify the user defined format for reading earthquake data.

The symbols in the read statement are kdate (A6), khrmn (A4), lat (integer degrees), "s" in blank (A1), xlat(real minutes), xdepth, xmag, quality (A1).

example; (A6, A4, I2, A1, F5.2, I3, A1, F5.2,2f7.2, 34x, A1)

line $5(12 x, A 60)$

station seg - This field is used to specify the segment containing the station data.

line $6 \quad(A 80)$

This line is used to specify the user defined station format

The variables in the read stations are

name (A4), lat (integer degrees), "s" in blank (A1), xlat

(decimal minutes), Ion (integer degrees), "en in blank

(A1), xlon (decimal minutes).

example format; (A4, I2, A1, I5.2, I3, A1, I5.2) 
line $7 \quad(10 x, 160)$

other seg - This field is used to specify the segment that the other data is in.

line 8 ( $(A 80)$

This line is used to specify the user defined format for the other data.

The variables in the read statement are:

name (A20), lat (integer degrees), "s" or blank

xlat (decimal minutes), lon (integer degrees), "en or blank, xlon (decimal minutes)

line $9(10 x, A 60)$

digit seg - This field is used to specify the segment used for the user defined boundary file when $\mathrm{mfil}=2$.

line $10 \quad(A B O)$

This is the format for the user defined boundary segment. variables in the read statement are (test (A3), idline (Alat, A lon)).

where test (A3) is used to test whether to lift the pen. idline is the number of (lat, lon) pairs on each line. ALAT and ALON are decimal deegrees. 
line $11(6 x, 45,7 x, 11,7 x, 11,6 x, 11,6 x, 11,5 x, 11,8 x, 11,7 x, 44)$ dproj - map projection

(cylin) 1 - cylindrical

(merca) 2 - mercator

(exact) 3 - exact cylindrical

(corre) 4 - corrected mercator

(mollw) 5 - molleweide

(aitof) 6 - aitoff

(sanso) 7 - sansom

(ellip) 8 - elliptical

(confo) 9 - conic conformal

(alber)10 - Albers conic equal area

(polyc) 11 - polyconic

(mypr2)12 - myproj2(transverse mercator)

(gnomo) 13 - gnomic

(ortho) 14 - orthographic

(stere) 15 - stereographic

(azimu)16 - Azimuthal equidistant

(lambe) 17 - lambert azimuthal equal area

(mypr3)18 - Transverse mercator sphericod (CAM UTM)

(mypr1)19 - Universal transverse mercator

$$
\begin{gathered}
\text { Ifram - frame for map } \\
0 \text { - no frame } \\
\text { 1-frame }
\end{gathered}
$$

mgrid - 0- no grid for map 
mfil 0 - no boundary file for map

1- Disspla map file

2- user defined map file

mref 0 - let Disspla pick reference parallels for conic projection 1-specify standard parallels for conic projection by yllat and yulat on line 12 .

mpol 0- point of tangency for azimuthal projections at $(0 ., 0$.

1- specify a point of tangency for azimuthal projections at point (xpole, ypole)

idline- for a user-defined boundary file (mfil $=2)$, this is the number of (lat-lon) pairs per line of input.

dmpda- for mfil = 1 , this is the Disspla boundary segment (A4)

1 - mapdta (mapd)

2 - hershey (hers)

3 - africa (afri)

4 - antarctic (anti)

5 - asia (asia)

6 - australia (aust)

7 - europe (euro)

8 - north america (nort)

9 - south america (sout)

10- pafrica (pafr)

11- pasia (pasi)

12- paustralia (paus) 


$$
\begin{aligned}
& \text { 13- peurope (peur) } \\
& \text { 14- pnorth america (pnor) } \\
& \text { 15- psouth america (psou) } \\
& 16 \text { - combined coastlines (coas) } \\
& 17 \text { - combined political (poli) } \\
& 18 \text { - low resolution usa (usal) } \\
& 19 \text { - medium resolution usa (usam) } \\
& 20 \text { - high resolution usa (usah) }
\end{aligned}
$$

line $12(6 x, f 8.3,7 x, f 8.2,7 x, f 8.3,7 x, F 8.3)$

xpole, ypole - with mpol $=1$, these define the point of tangency for azimuthal projection in decimal degress

$$
\text { for example: } \quad 30.95,-121.37
$$

yllat, yulat - with mref $=1$, these define the user-specified standard parallels for conic projections in decimal degrees

1.e. i.e. $(+45.90,+65.10)$

line $13(6 x, f 8.3,6 x, f 8.3)$

ympol, xmpol - for dproj = "mypro", these specify the cylinder axis direction for the transverse mercator in decimal degrees

ympol should be equal to 0.0 for cylinder axis on equator. xmpol is the central longitude for the projection.

line $14(6 x, f 10.5,7 x, f 10.5,7 x, f 10.5,7 x, f 10.5)$ alono, alato - longitude and latitude of the point $A$ in decimal degrees 
42

alonp, alatp - longitude and latitude of the point $A^{\prime}$ in decimal degrees

These points help define the workbox used for all plots other than maps.

line $15 \quad(6 x, F 7.3,6 x, I 1,7 x, F 10.3)$

enaap - height of symbols $A-A^{\prime}$ on map in inches 1.e. .21 inches naap - 0 no symbols $A, A^{\prime}$ on map

1 draw symbols $A, A^{\prime}$ on map

Xwid1 - This is the left most distance from $A-A^{\prime}$ used to define the workbox.

Xwid2 - This is the right most distance from $A-A^{\prime}$ used to define the workbox.

line $16(8 x, f 4.2,1 x, f 4.2,13 x, 440)$

hite(1) - This is height of the main heading in inches.

hite(2) - Height of the subheading and axis labels in inches.

main heading - This is the main heading for the plot. This must be centered in the 40 character field in order to be centered on the plot.

Iine 17 (16x, A40)

subheading - This is the subheading for the plot. It must be centered in the 40 character field in order to be centered on the plot. 
Iine $18(6 x, f 10.3,7 x, f 10.3)$

xaxis, yaxis - These define the plot size in inches.

For maps, the plot is expanded to fill the y-axis in inches. The $x$-axis must be large enough to avoid chopping the latitude range.

For other plots, the $\mathrm{x}$ and $\mathrm{y}$ axis scale independently.

line $19(6 x, e 15.8,6 x, e 15.8,6 x, e 15.8)$

line $20(6 x, e 15.8,6 x, e 15.8,6 x, 315.8)$

line 19 xorig, xstp, xmax

line 20 yorig, ystp, ymax

These are the plot limits and step sizes in axis units. The meaning of these will be explained briefly for each type of plot.

a. maps These variables will be decimal degrees. where

$$
\begin{aligned}
& \text { xorig - left most longitude } \\
& \text { xstp - longitude increment } \\
& \text { xmax - right most longitude } \\
& \text { yorig - lower latidude } \\
& \text { ystp - latitude increment } \\
& \text { ymax - upper latitude }
\end{aligned}
$$

An integral number of intervals should fit in the latitude and longitude range.

b. cross sections For cross sections along A-A': xorig $-0.0 \mathrm{~km}$ (at point A). need not be given xstp - xaxis increment in $\mathrm{km}$ 
$x \max$ - $x x \mathrm{~km}$ where $x x$ is the distance in $\mathrm{km}$ between $A$ and $A^{\prime}$. This is calculated in maplot2 and need not be given. For perpendiculer views of A-A': xorig - (xwid1) in $\mathrm{km}$. Need not be specified here. xstp - $x$-axis increment in $\mathrm{km}$ $x \max$ - xwid2 in $\mathrm{km}$. Need not be specified here.

for both type of cross sections:

yorig - xdep1 in $\mathrm{km}$. Need not be specifled here. ystp - depth increment in $\mathrm{km}$.

ymax - xdep2 in $\mathrm{km}$. Need not be specified here. c. time distance plots The $x$-axis is in time from the beginning time to the ending time as given on line 27. The time units are also specified on line 27.

The $x$-axis goes from 0.0 to TT in user defined time units. xorig and $x \max$ need not be specified here. The time increment, xstp, in user time units mast be specified. (1.e. 5 months)

The $y$-axis goes along $A-A^{\prime}$ from $0.0 \mathrm{~km}$ to $x \mathbf{k m}$ at point $A^{\prime}$. yorig and ymax need not be given, but ystp, the distance increment in $\mathrm{km}$, must be specified (i.e. $2 \mathrm{~km}$ ) 
d. magnitude time plots The $x$-axis is time from 0.0 to $T$ in user defined time units. xorig and xmax need not be specified. The time increment must be specified (i.e. 5 days). The y-axis is magnitude and goes from xmag 1 to xmag2 as specified on line 21. Yorig and ymax need not be specified, but ystp, the magnitude increment, must be specified.

e. magnitude-frequency plot This is a semi-log plot. The $x$-axis goes from xmag 1 and xmag2 as specified on line 21. Xorig and $x \max$ need not be specified, but the magnitude increment xstp, must be specified. The $y$-axis is log-number. The smallest number, yorig, must be specified (i.e., 1.).

ystp is the number of cycles on the y-axis (i.e. 2 cycles). Ymax is not used here.

f. cumulative moment plot The $x$-axis is time from 0.0 to IT in used defined units. Xorig and $x \max$ need not be specified, but xstp, the time increment, must be specified.

The $y$-axis is cumulative seismic moment in dyne-cm. This axis is self-scaling to the data. Thus, yorig, ystp, and ymax need not be specified.

B. histogram plots The $x$-axis is time, distance, or depth. Xoris and ymax need not be specified. Xstp must be specified as the increment in the relevant axis units. The y-axis is either number or summed moment. This axis is self scaling. Thus, yorig, ystp, and ymax need not be specified. 
h. time-frequency plot This is a log-log plot. The start time after the beginning time must be given, xorig (i.e. .001). This must be given in user defined time units. xstp is the number of cycles to draw on the time axis.

Yorig is the smallest frequency (greater than 0.0) for the y-axis. Istp is the number of Log cycles to draw (i.e. 2.).

line $21(6 x, f 6.3,7 x, f 6.3,8 x, f 6.3)$

xmag 1 - this is the small magnitude cutoff

xmag2 - This is the large magnitude cutoff where $x \operatorname{mag} 16 \operatorname{mag}<x \operatorname{mag} 2$

delmag - This is the magnitude increment starting from xmag 1 used for different symbol types scaled with magnitude.

line $22(6 x, f 7.2,7 x, f 7.2,8 x, f 7.2)$

xdep1 - this is the shallow depth cutoff

xdep2 - this is the deep depth cutoff where $x \operatorname{dep} 1 \leqslant \operatorname{depth}<\mathbf{x d e p} 1$

deldep - This is the depth increment starting from xdep1 used for symbol type 3 which has a different marker for each depth Increment.

line $23(7 x, 11,8 x, 11,8 x, 11)$

lestyp - type of earthquake symbol

1 - symbol with no scaling

2 - symbol with magnitude scaling 
3 - different symbol with each depth increment scaled with magnitude

4 - a number representing magnitude - no scaling

5 - a number or letter representing depth down to $35 \mathrm{~km}$, scaled with magnitude

6 - a letter representing quality, scaled with magnitude isstyp - type of station symbol

1 - symbol with no label

2 - symbol with station name to the upper right lostyp - type of OTHER symbol

1 - symbol with no label

2 - symbol with name to the upper right

line $24(5 x, f 7.4,7 x, f 7.4,5 x, 11)$

esiz - a) for earthquake symbol with no scaling, this is the symbol size in inches (1.e. .14 inches)

b) for symbol with magnitude scaling, this is the size of the smallest magnitude symbol allowed.

esdel - a) for symbol with no magnitude scaling this is ignored b) for symbols with scaling, this is the increase in size between two consecutive magnitude increments (i.e. 0.5 inches)

1em - This is an integer between 0-14 which signifies the marker type. (see figure 2).

line $25(5 x, 77.4,6 x, 77.4,5 x, 12)$

ssiz - station symbol size in inches (i.e. .14 inches) 
slet - lettering size for station name (1.e. .14 inches)

ism - this is an integer between $0-14$ which signifies the marker type (see fig. 2)

line $26 \quad(5 x, f 7.4,6 x, 77.4,5 x, 12)$

osiz - The OTHER marker size in inches (1.e. .14 inches)

olet - lettering size for the OTHER name (1.e. .14 inches)

ism - this is an integer between $0-14$ which signifies the marker type. (see fig. 2)

line $27(7 x, A 6,8 x, 24,8 x, a 6,8 x, a 4,7 x, 11)$

itmind-(yr, month, day) for beginning time

itminh-(hr, min.) for beginning time

Itmaxd-(yr, month, day) for ending time

1tmaxh-(hr, min.) for ending time

For all plot other than time plots, these are ignored.

itime - user defined time units for time axis labeling

$$
\begin{aligned}
& 1 \text { - hours } \\
& 2 \text { - days } \\
& 3 \text { - months (approx) } \\
& 4 \text { - years (approx) }
\end{aligned}
$$

Iine $28(6 x, 86.3,7 x, 01,7 x, f 8.4,6 x, 86.3,5 x, 11,8 x, 11)$

This line is for the magnitude frequency plots. For all other plots, this line is ignored.

xbstp - magnitude increment for summing earthquakes

ibsym - a marker between 0-14 for plot symbol (see fig. 2)

bsize - marker size in inches (re .14 inches) 
smag - smallest magnitude used for calculation of b-value $1 b v$ -

$$
\begin{aligned}
& 0 \text { - cumulative frequency with no calculation of b-value } \\
& 1 \text { - cumulative frequency with calculation of b-value } \\
& 2 \text { - incremented density plot }
\end{aligned}
$$

Ibgrid -

0 - no grid for mag-frequency plot

1 - grid for mag-frequency plot

line $29(6 x, f 10.3,7 x, f 10.3,7 x, 11,9 x, f 10.3)$

This line is ignored for all plots other than cumulative moment and histogram plots.

aseis - "a" value in magnitude moment formula

bseis - "b" value in magnitude-moment formula

$$
\log M=a+b \text { (Mag) }
$$

iseis - for histogram plot

1 - earthquake number histogram

2 - summed moment histogram

xmagmin - minimum magnitude in conversion from magnitude to moment

line $30 \quad(7 x, 11,8 x, f 10.3)$

This line is ignored for all plots other than histograms and frequency time plots

itimax - for histogram plots, plot number of events with:

$$
\begin{aligned}
& 1 \text { - time } \\
& 2 \text { - distance along } a-a^{\prime} \\
& 3 \text { - perpendicular distance from } a-a^{\prime}
\end{aligned}
$$


4 - depth

xaxinc -

a) for histograms, increment on the $x$-axis for suming earthquakes in the appropriate units

b) for frequency time plots, the fraction of a $\log$ cycle in time used in summing earthquakes. 


\section{Bibliography}

AKI, K, 1965: Maximum Likelihood estimate of $b$ in the formula $\log N=$ $a-b M$ and 1 ts confidence limits. Bulletin of the Earthquake Research Institute, Vol. 43 pp. 237-239.

UTSU, T., 1961: A statistical study on the occurrence of aftershocks. Geophysical Magazine, vol. 30, no. 4, 521-605.

THATCHER, K., and HANKS, T., 1973: Source parameters of Southern California earthquakes. J. Geophys. Res., Vol. 78, 8547-8576. 


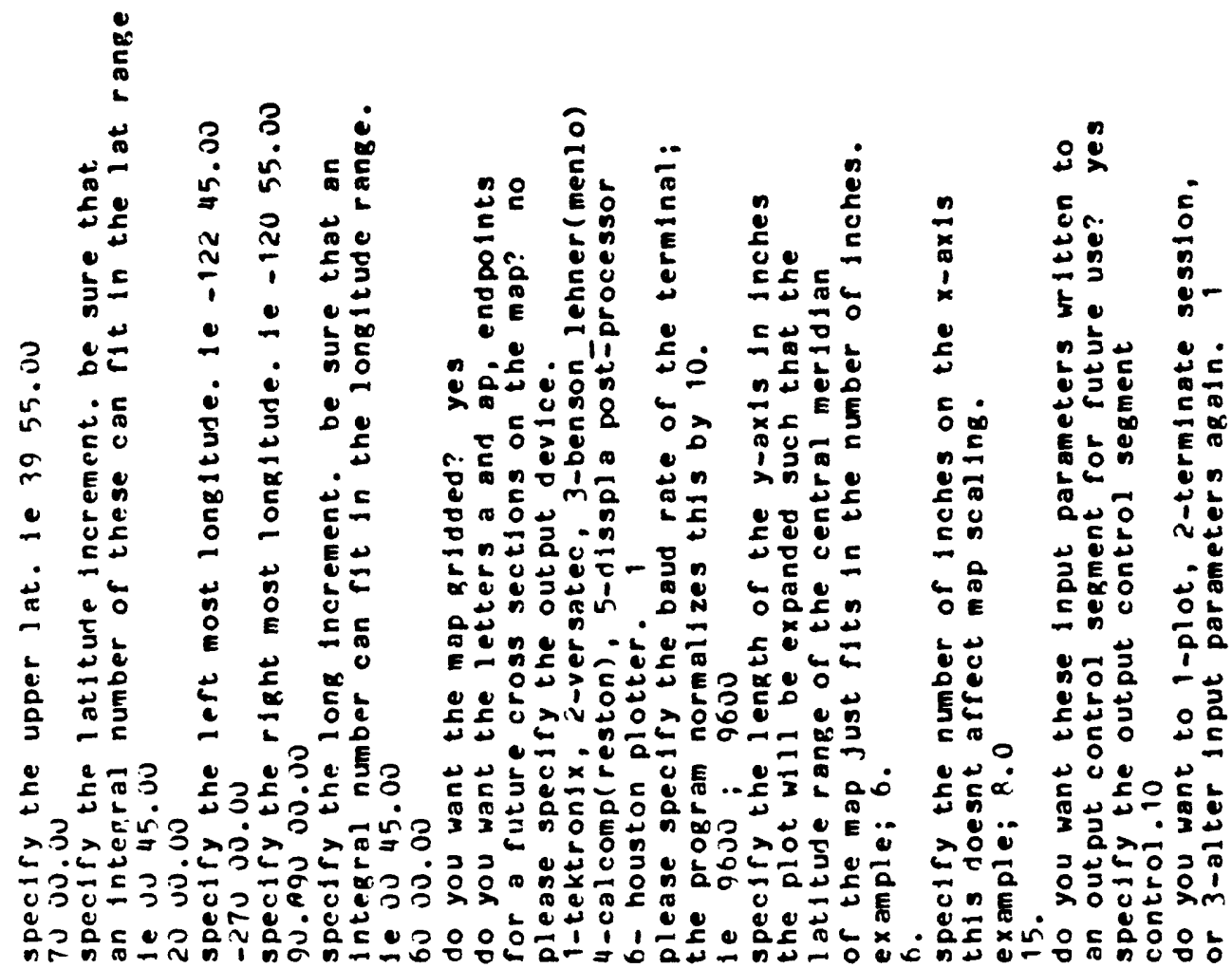

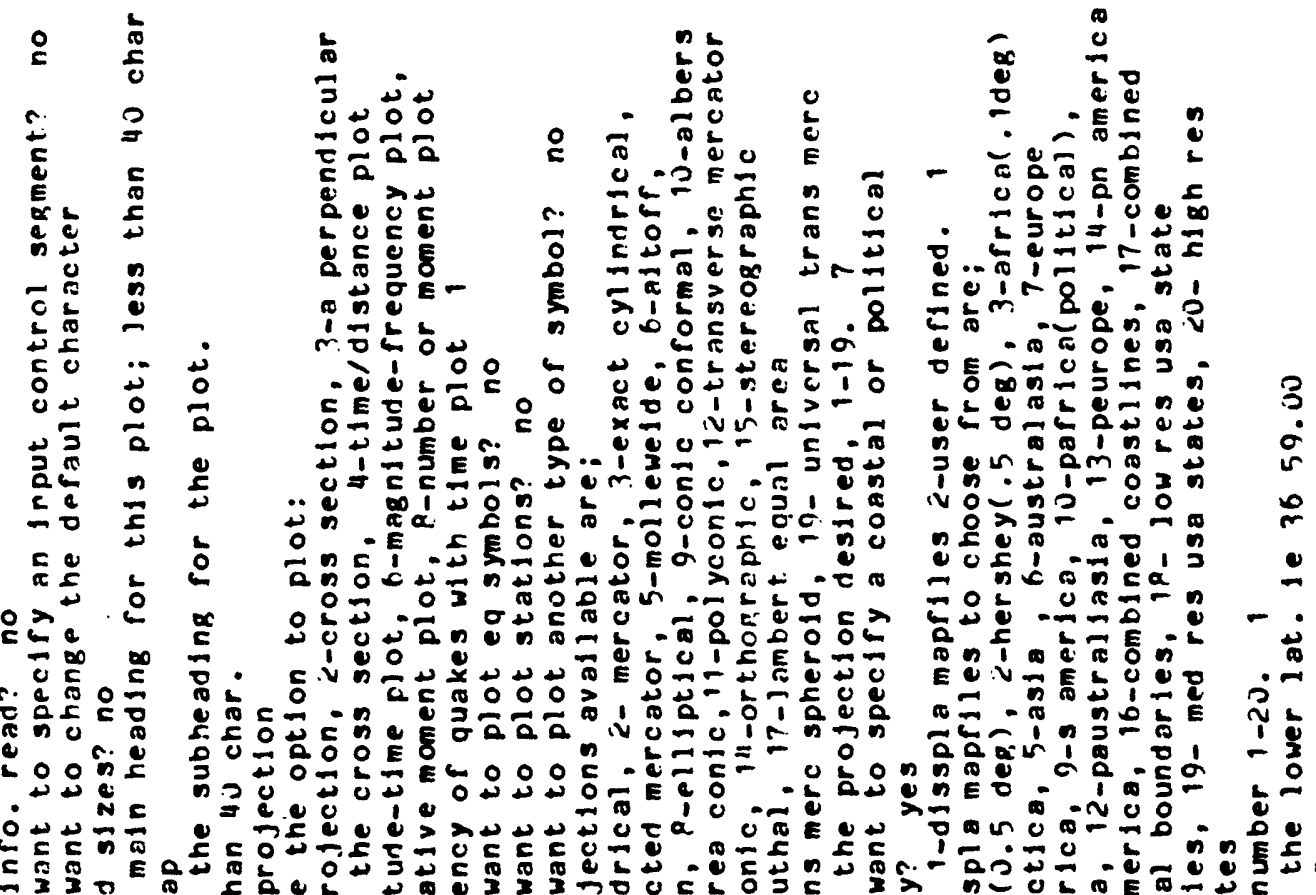
U J I I

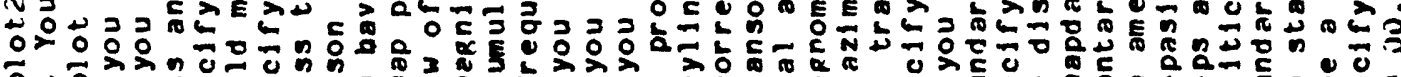

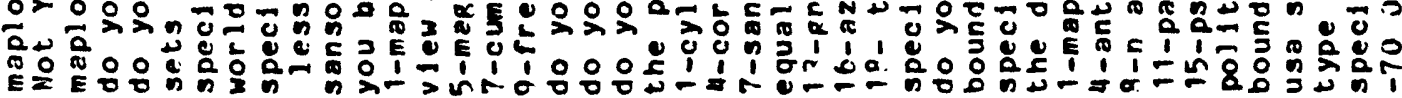



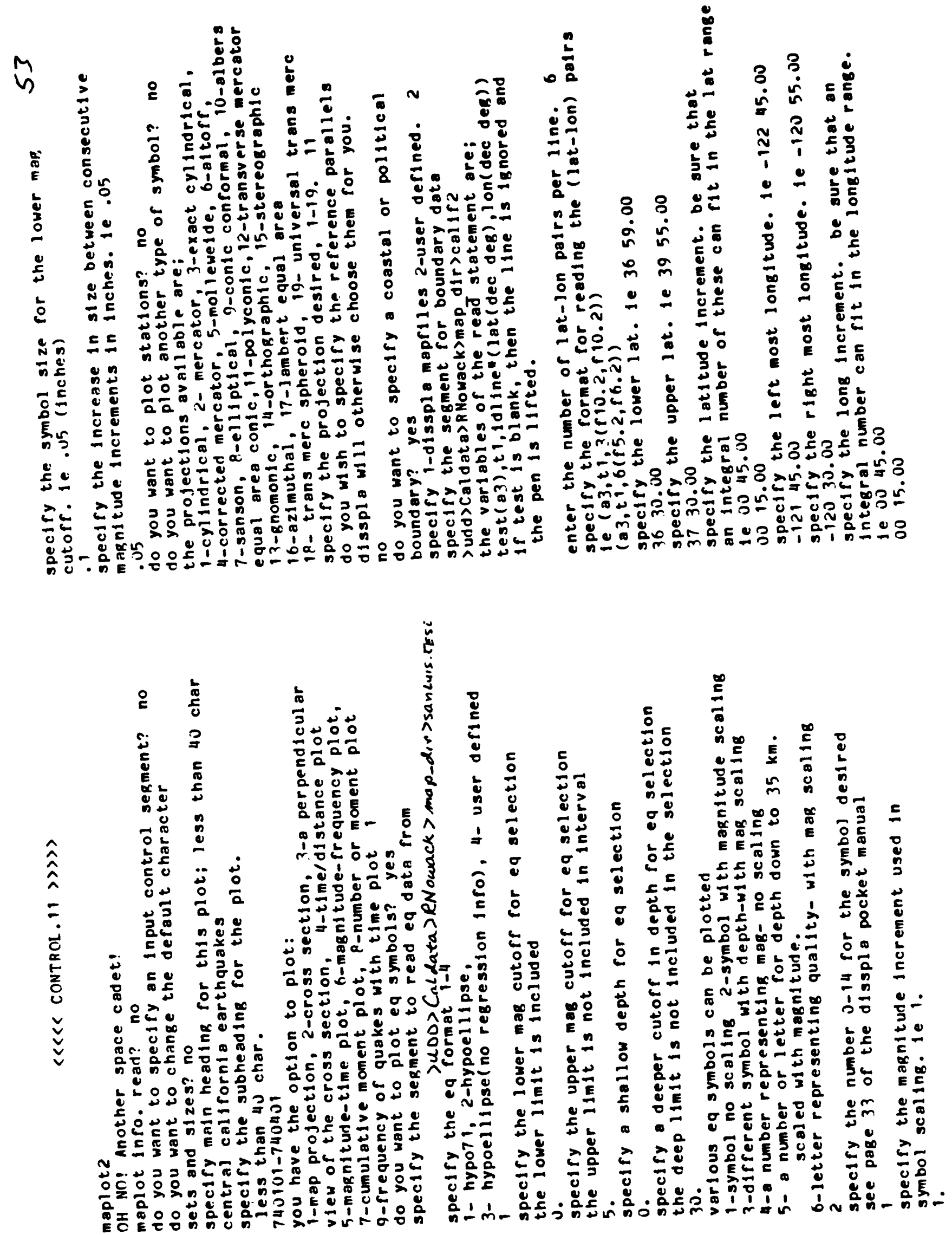


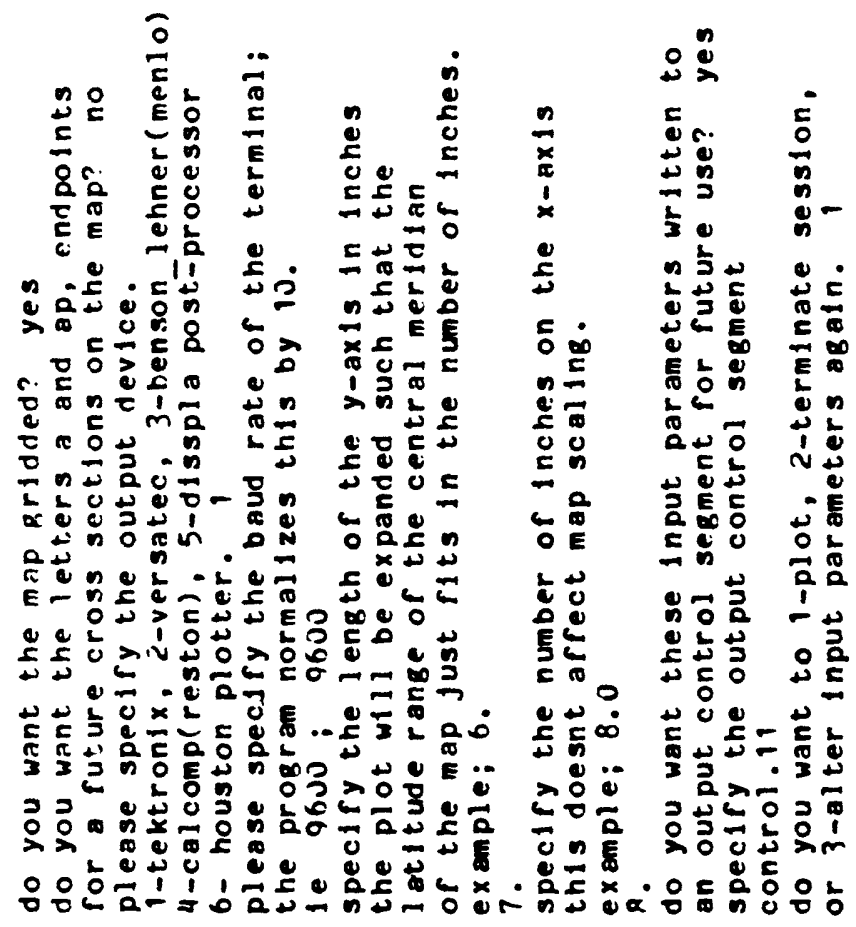


$n$

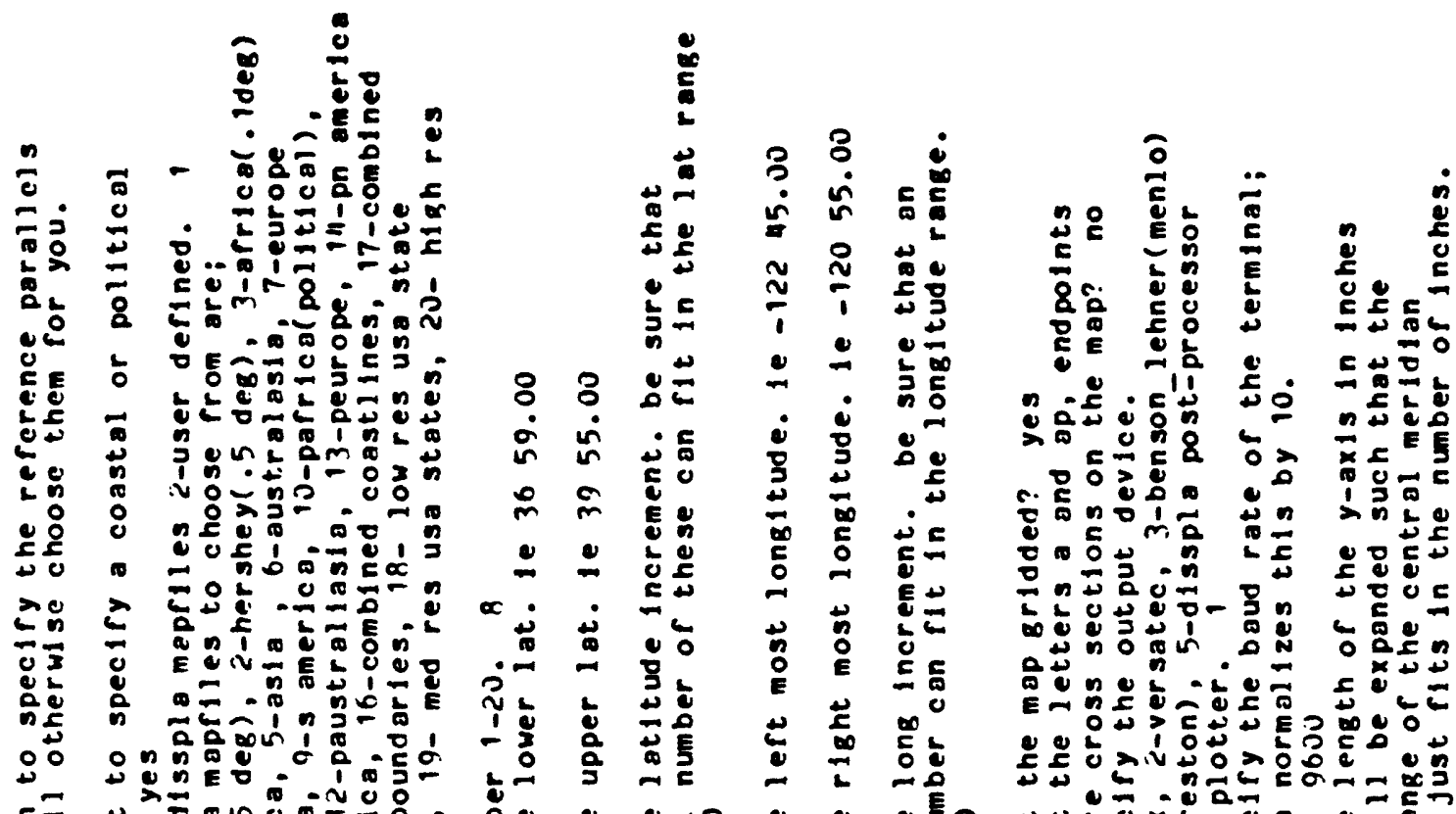

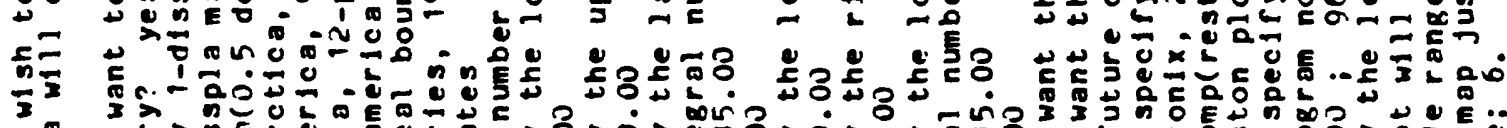

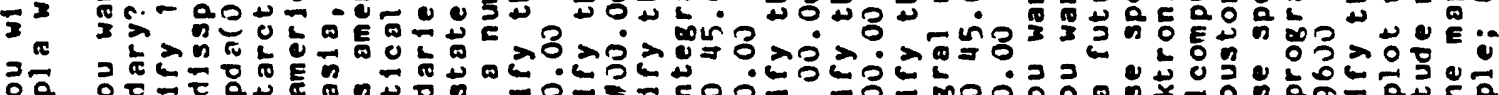

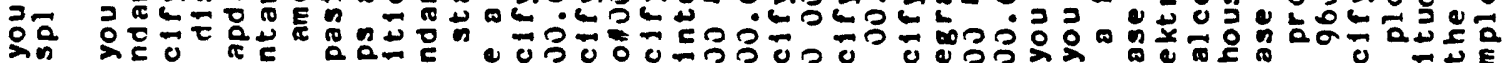

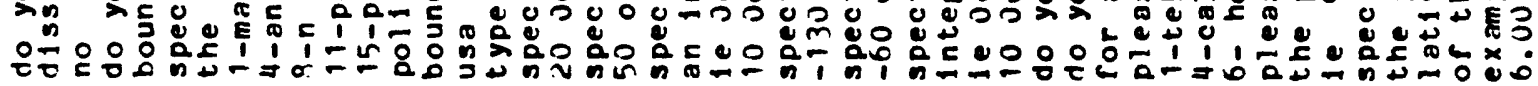

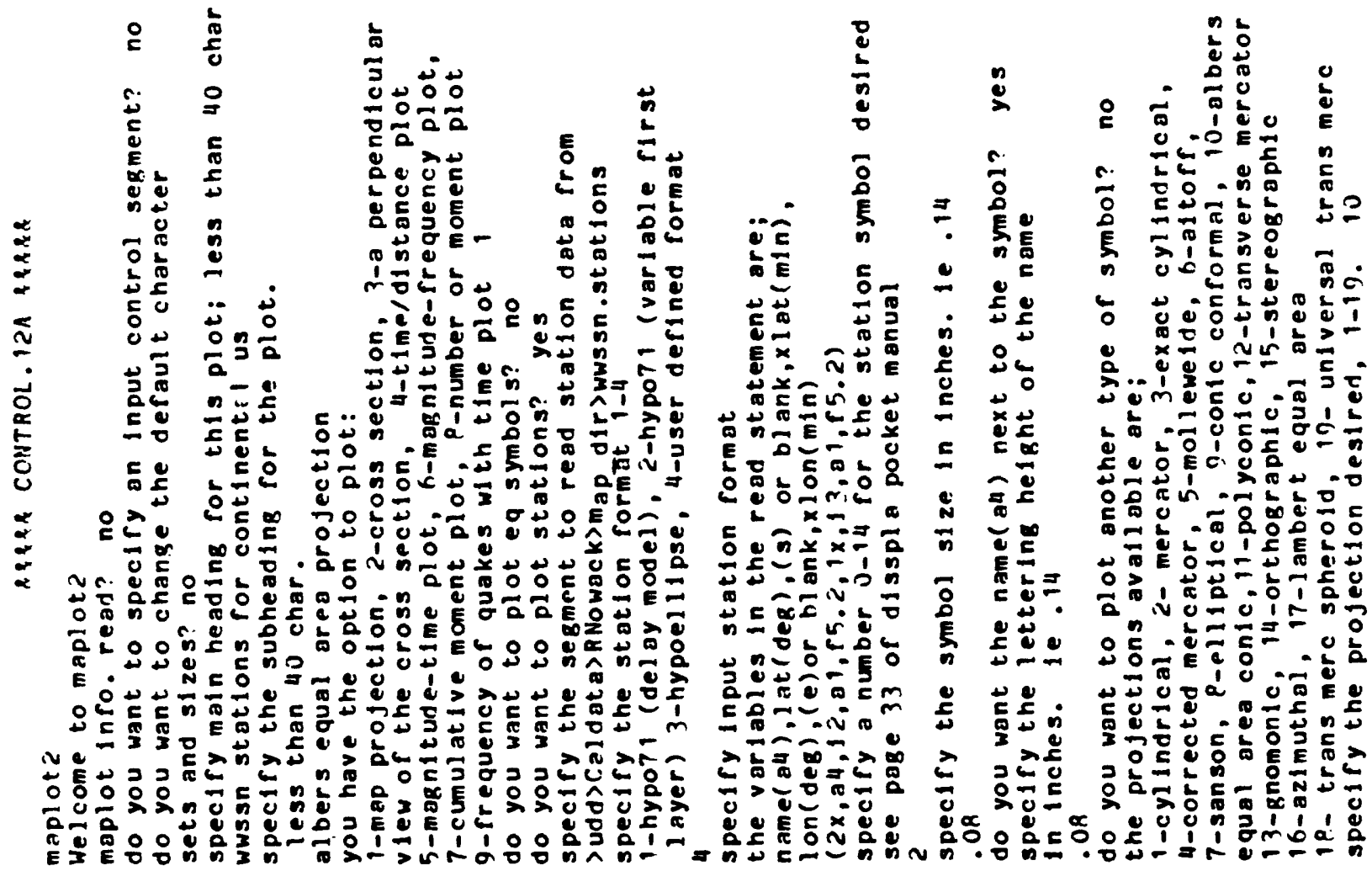




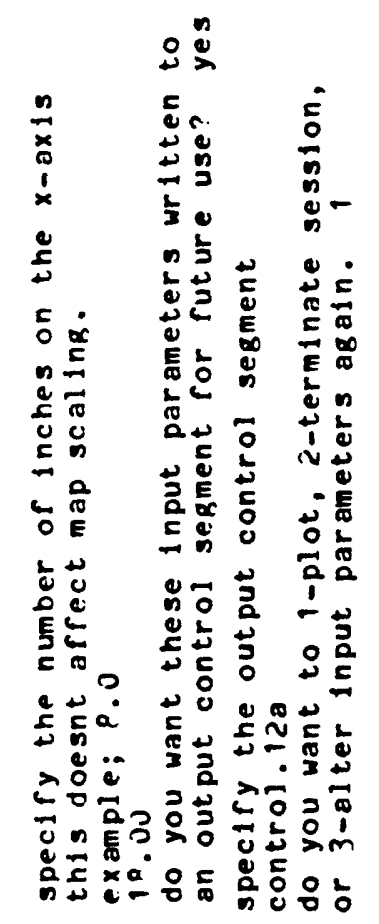


5

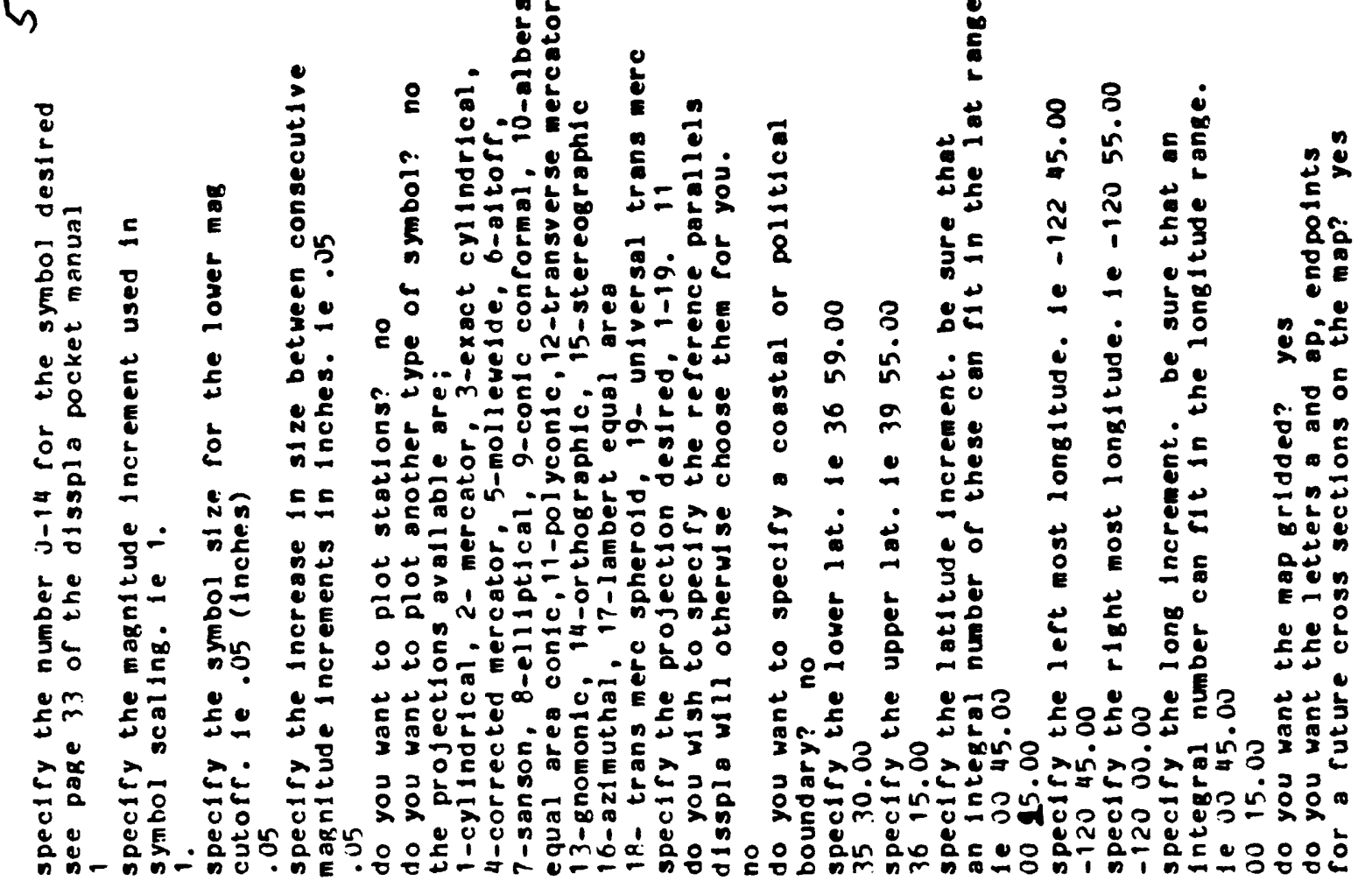

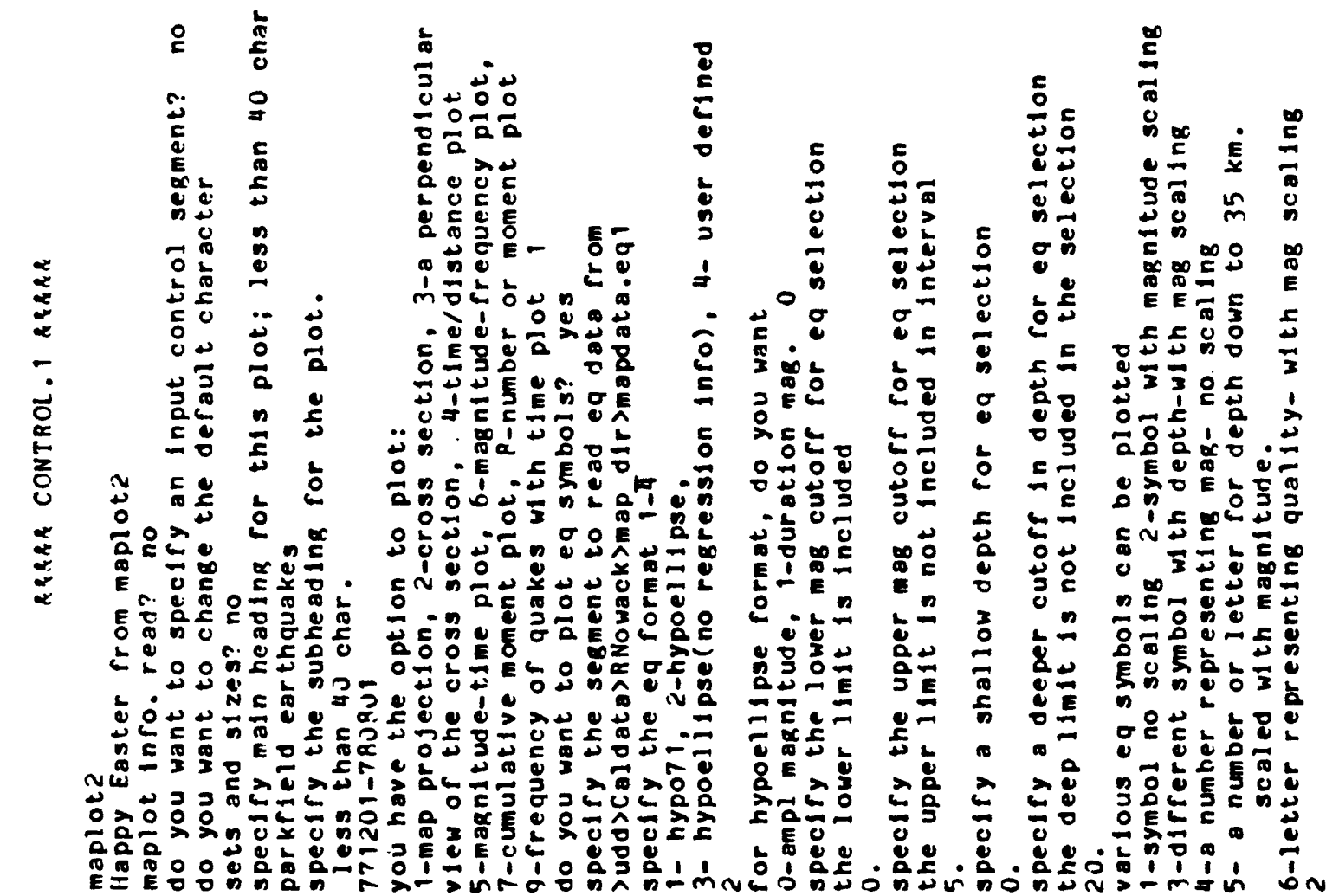




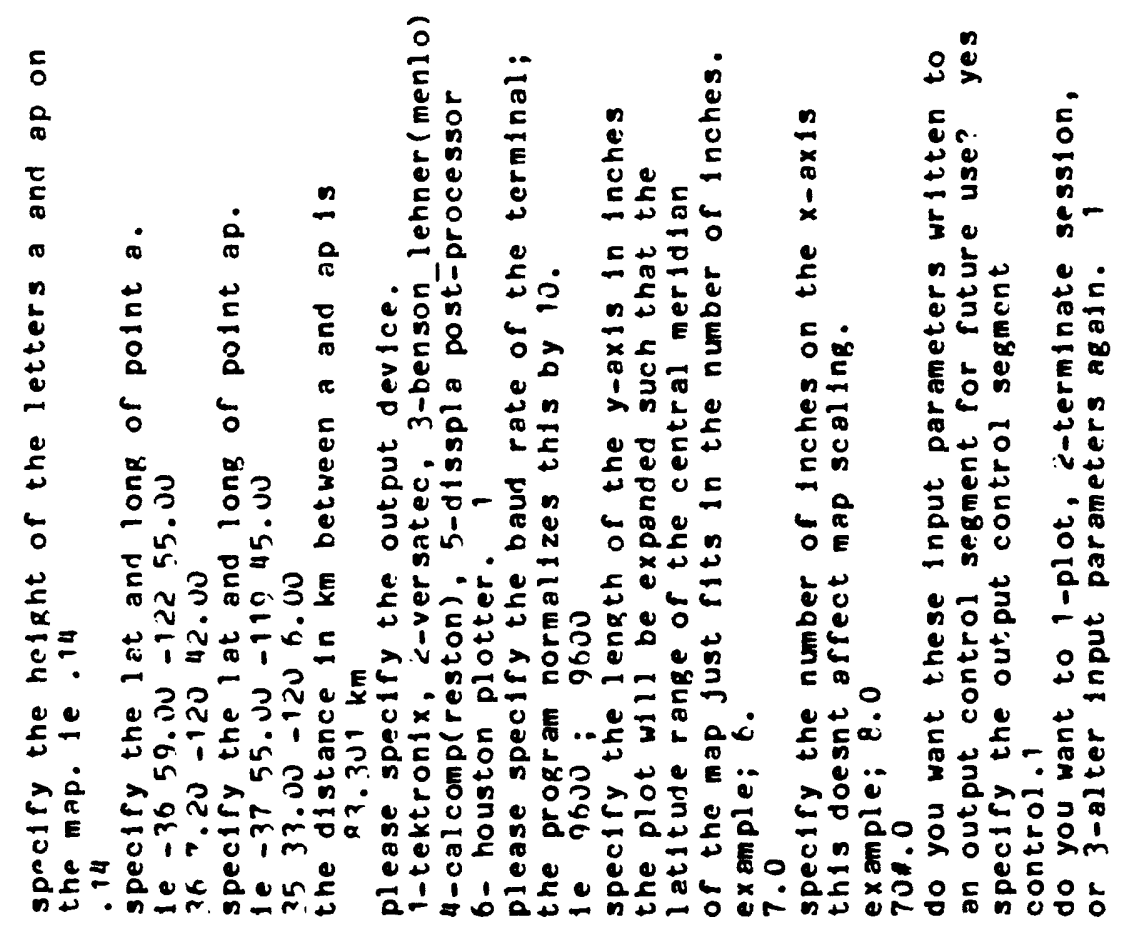


o
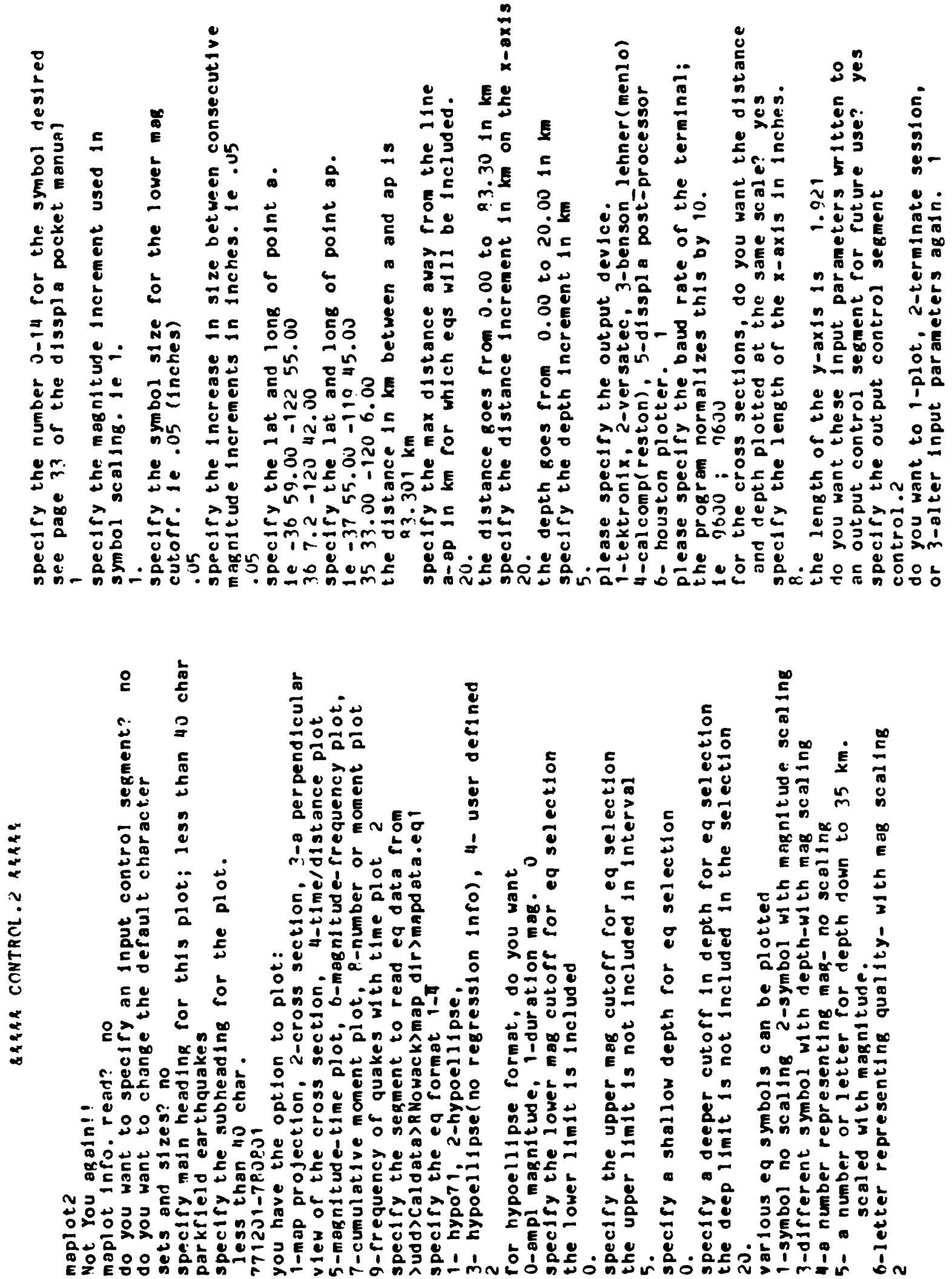
6
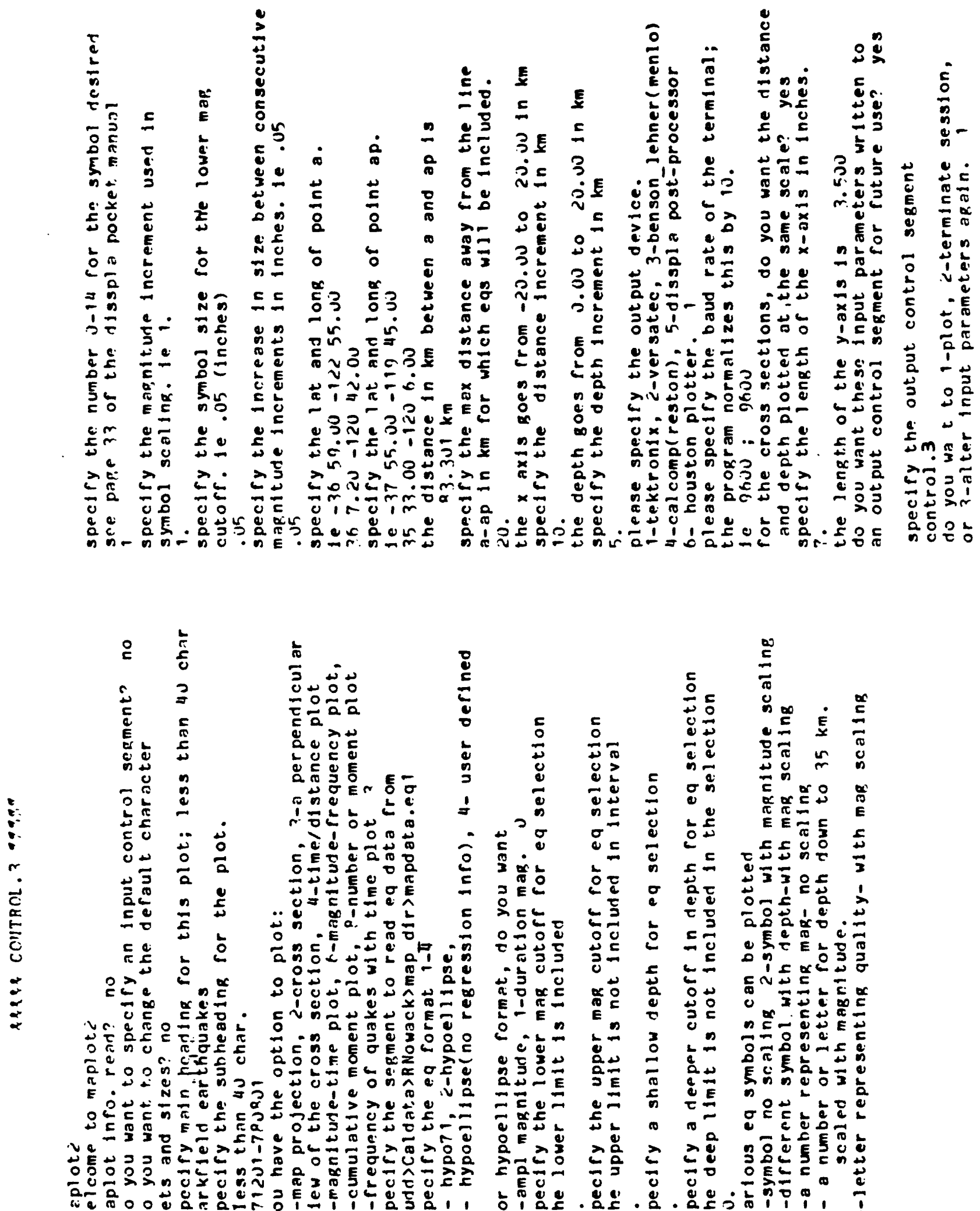


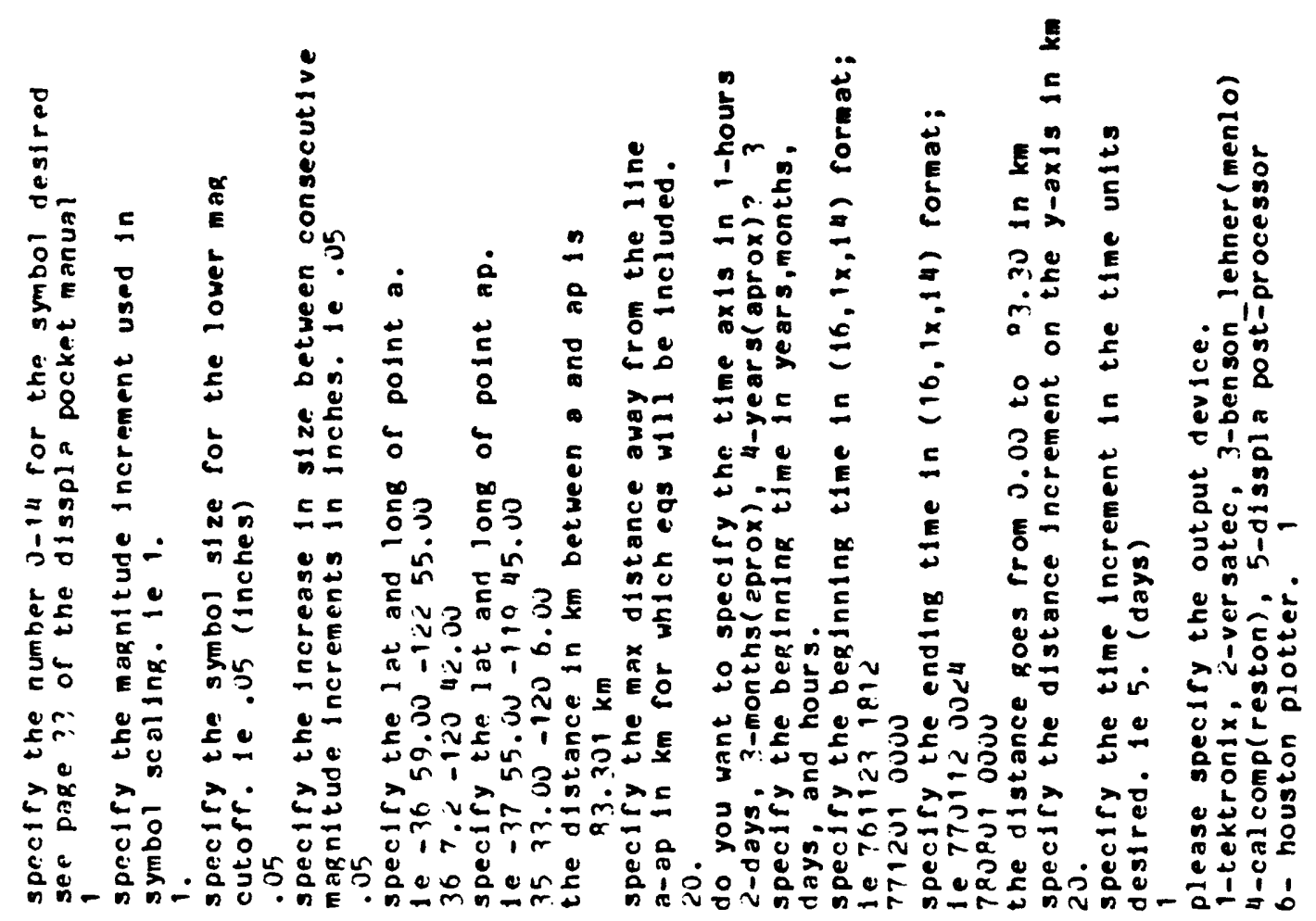

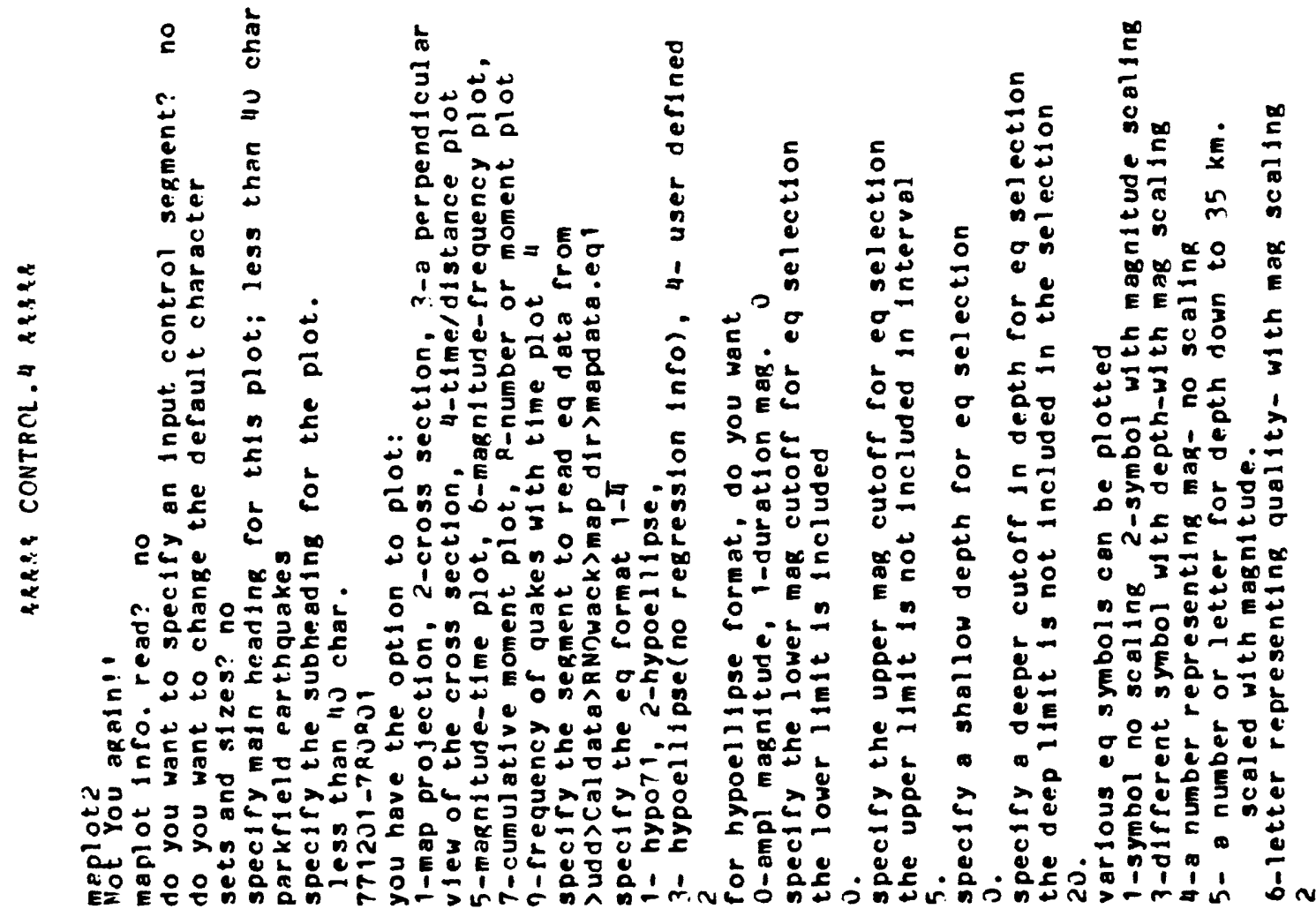


J

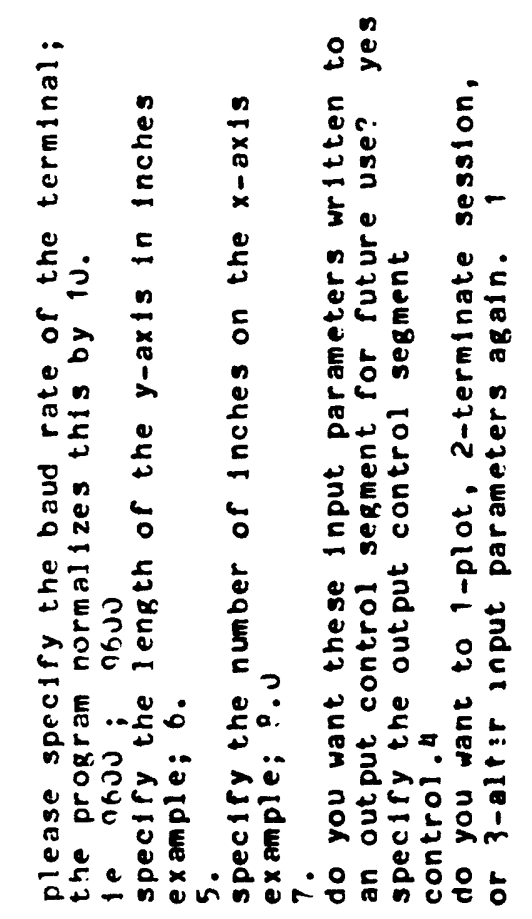




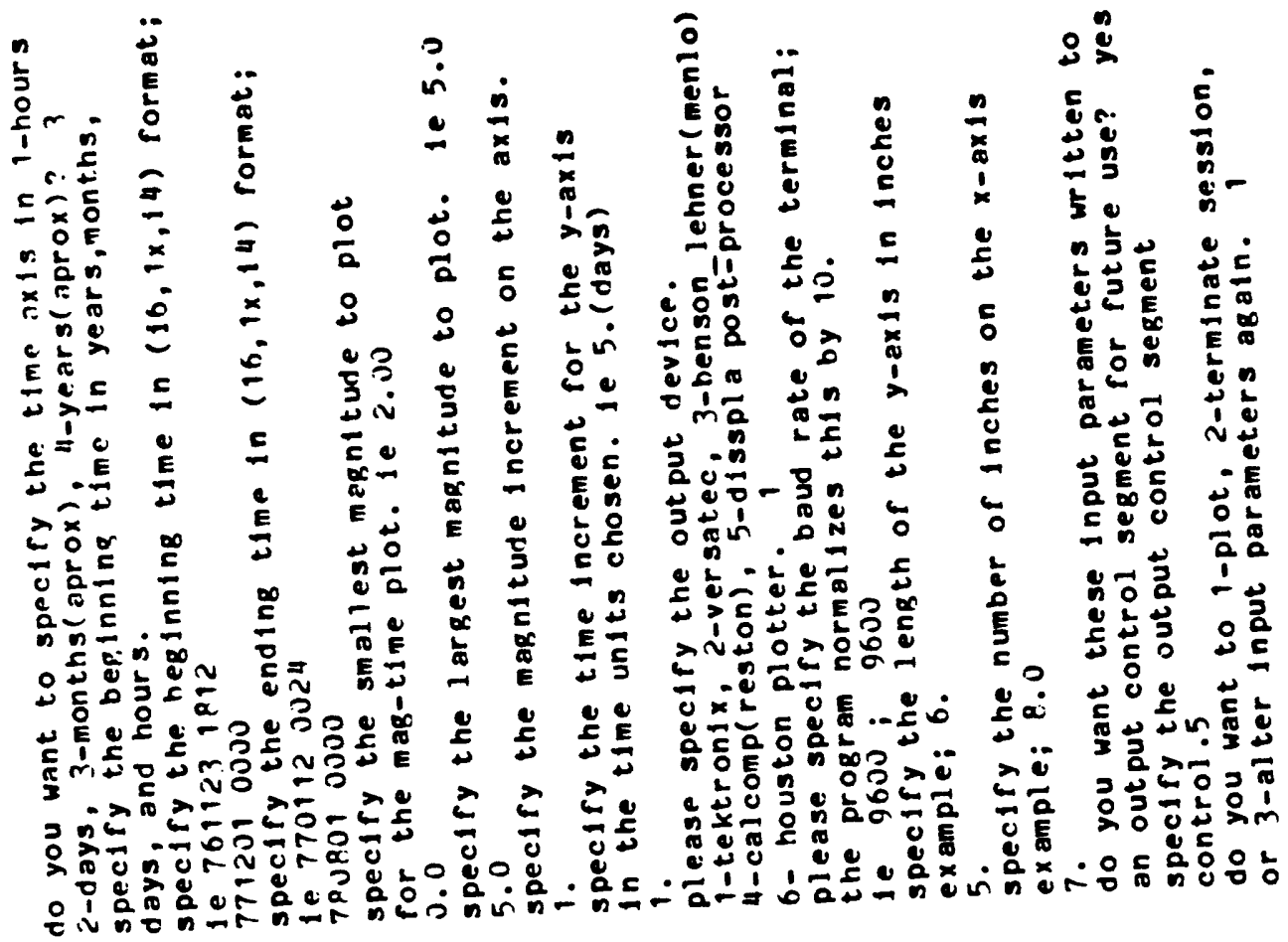

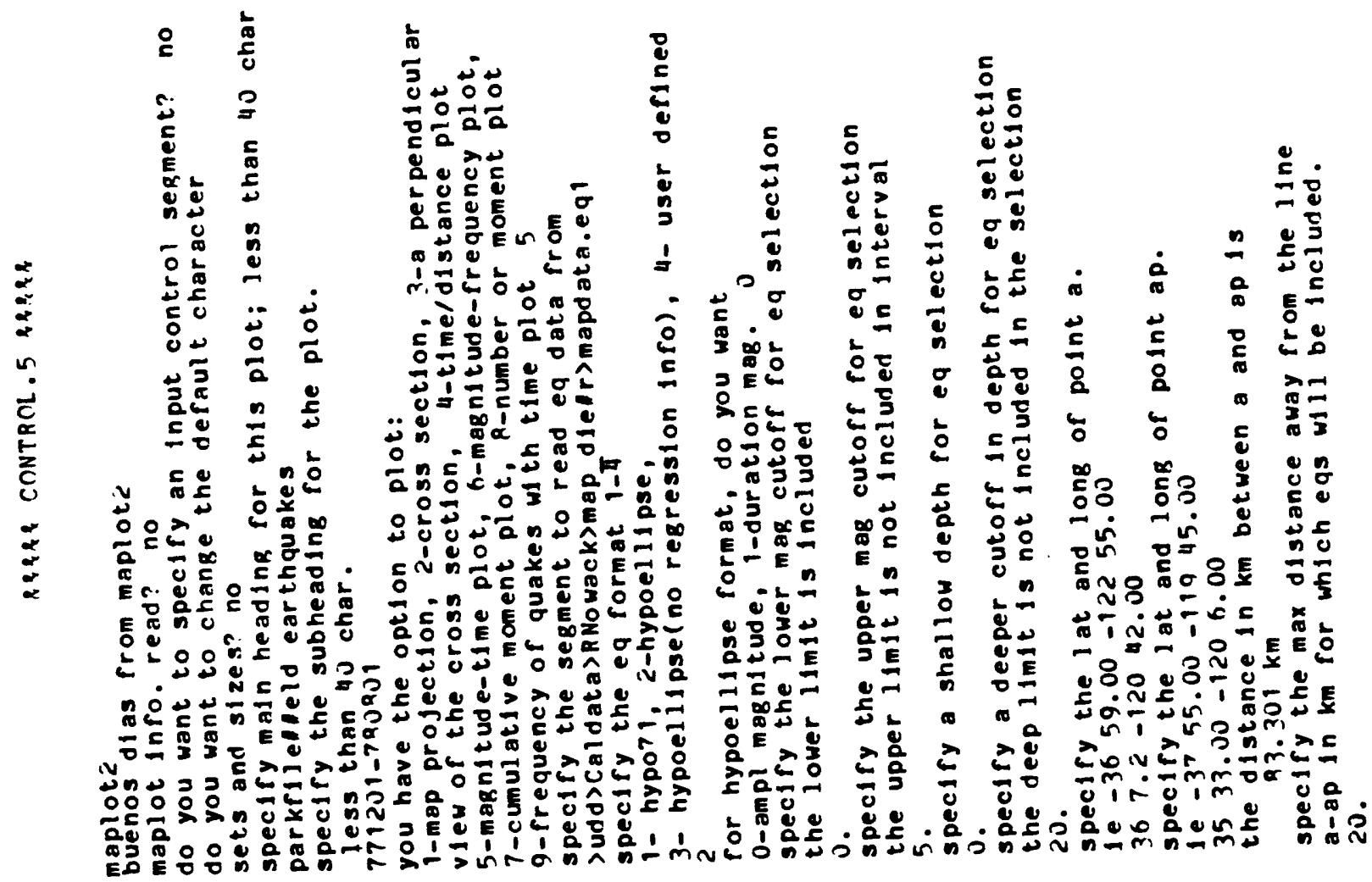



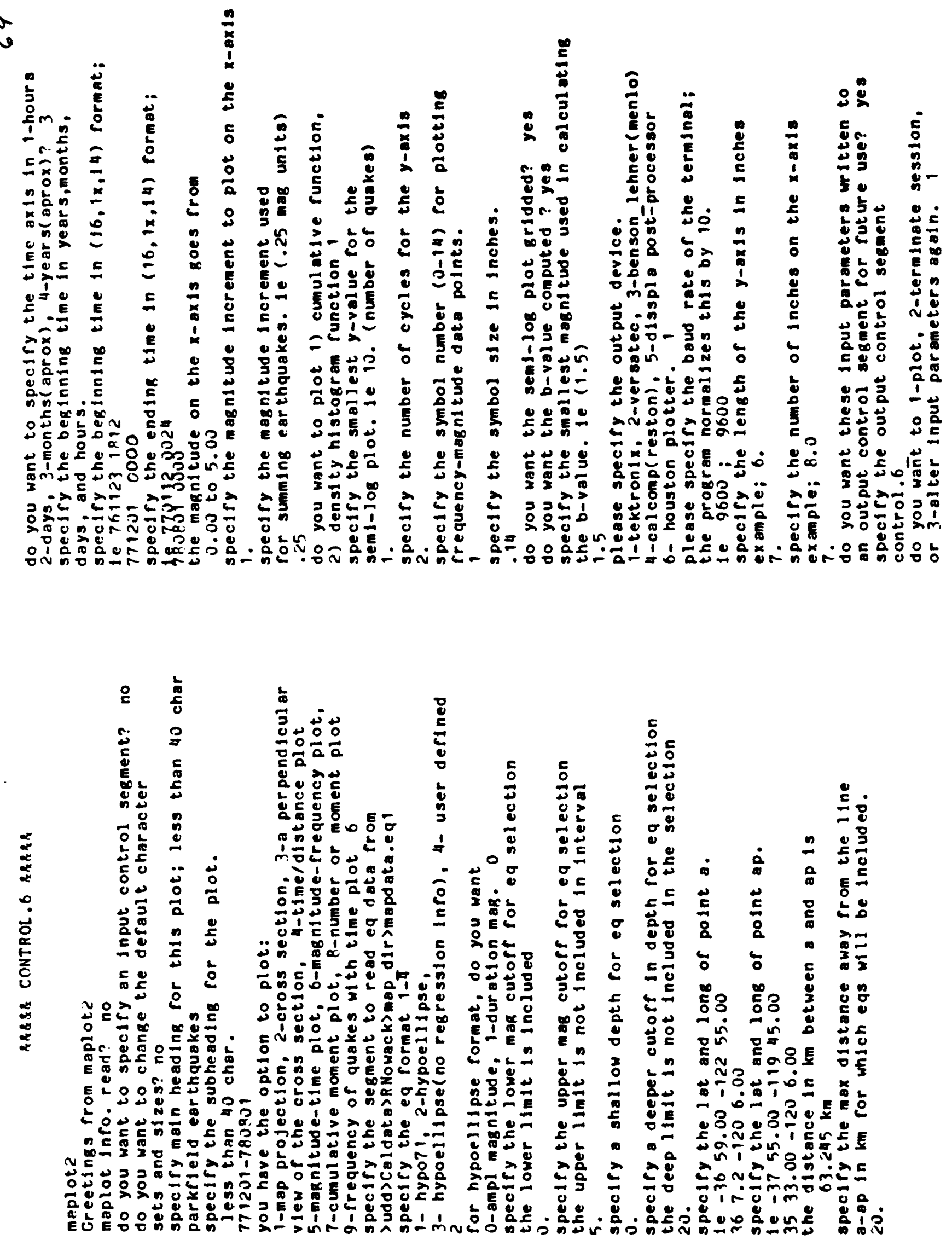


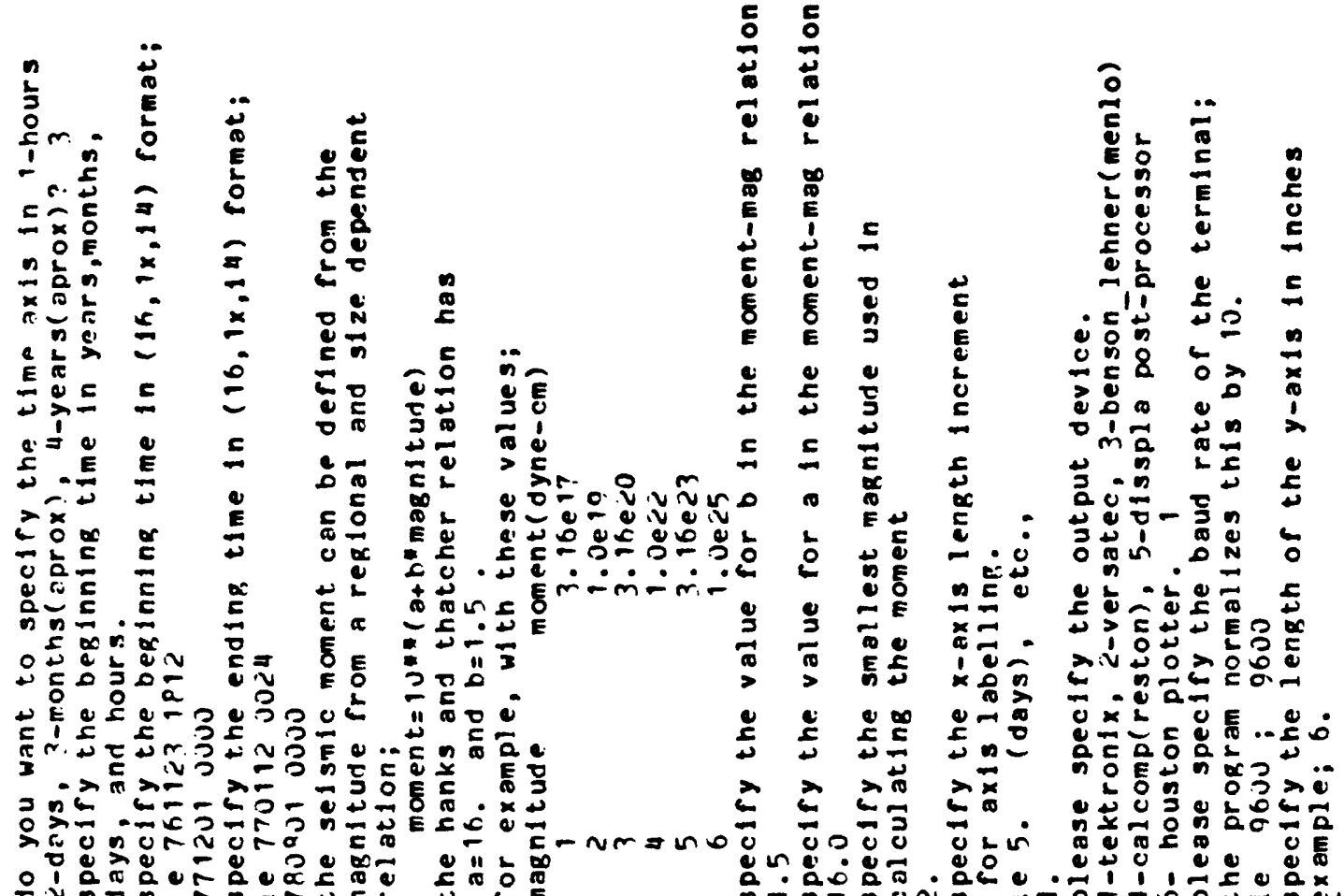

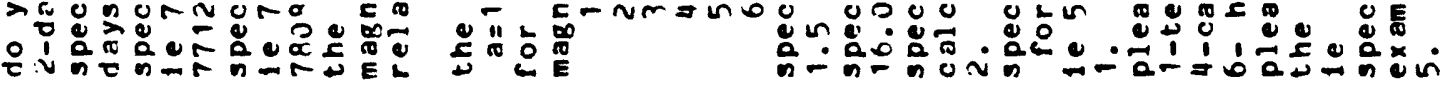

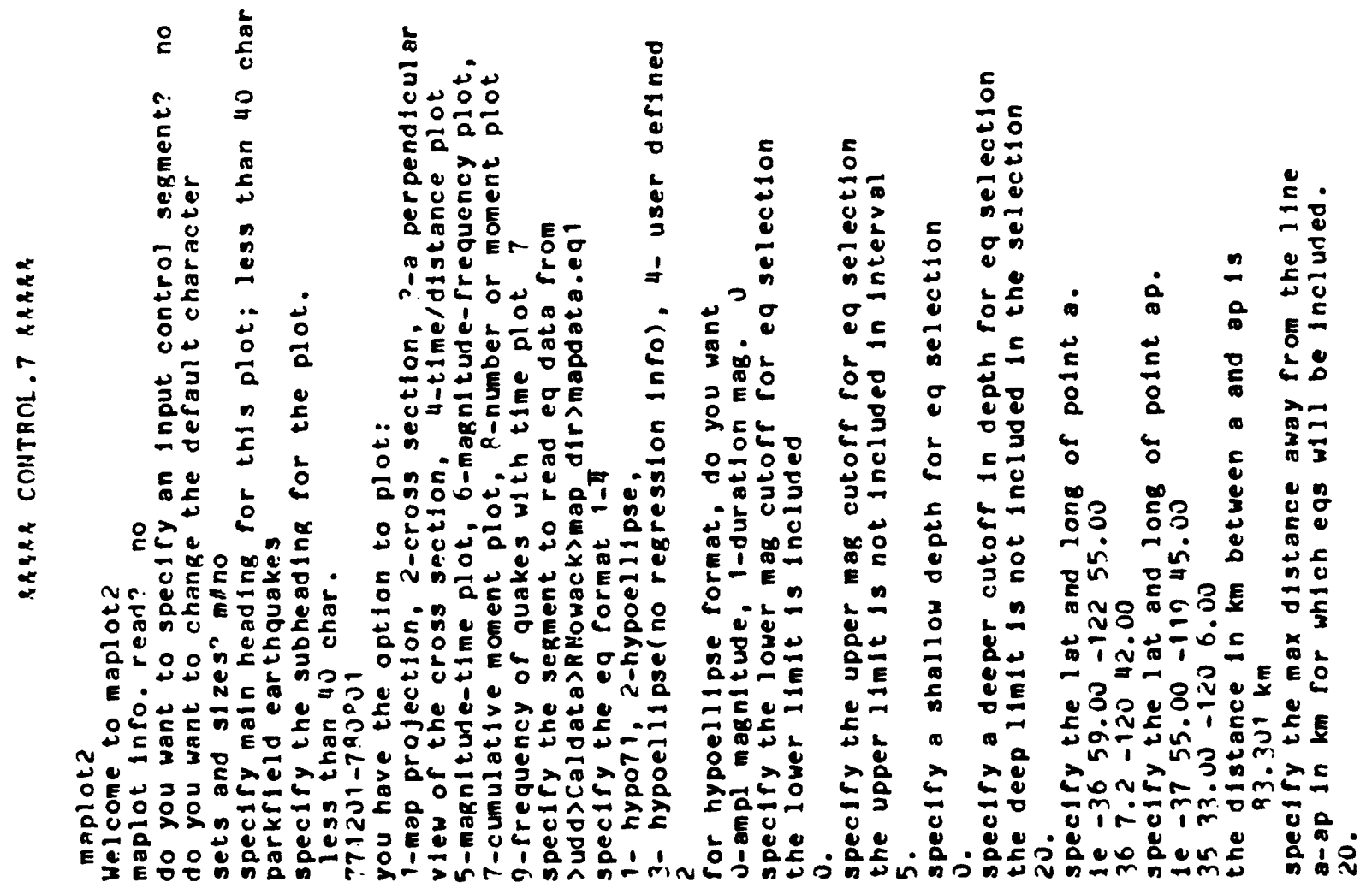



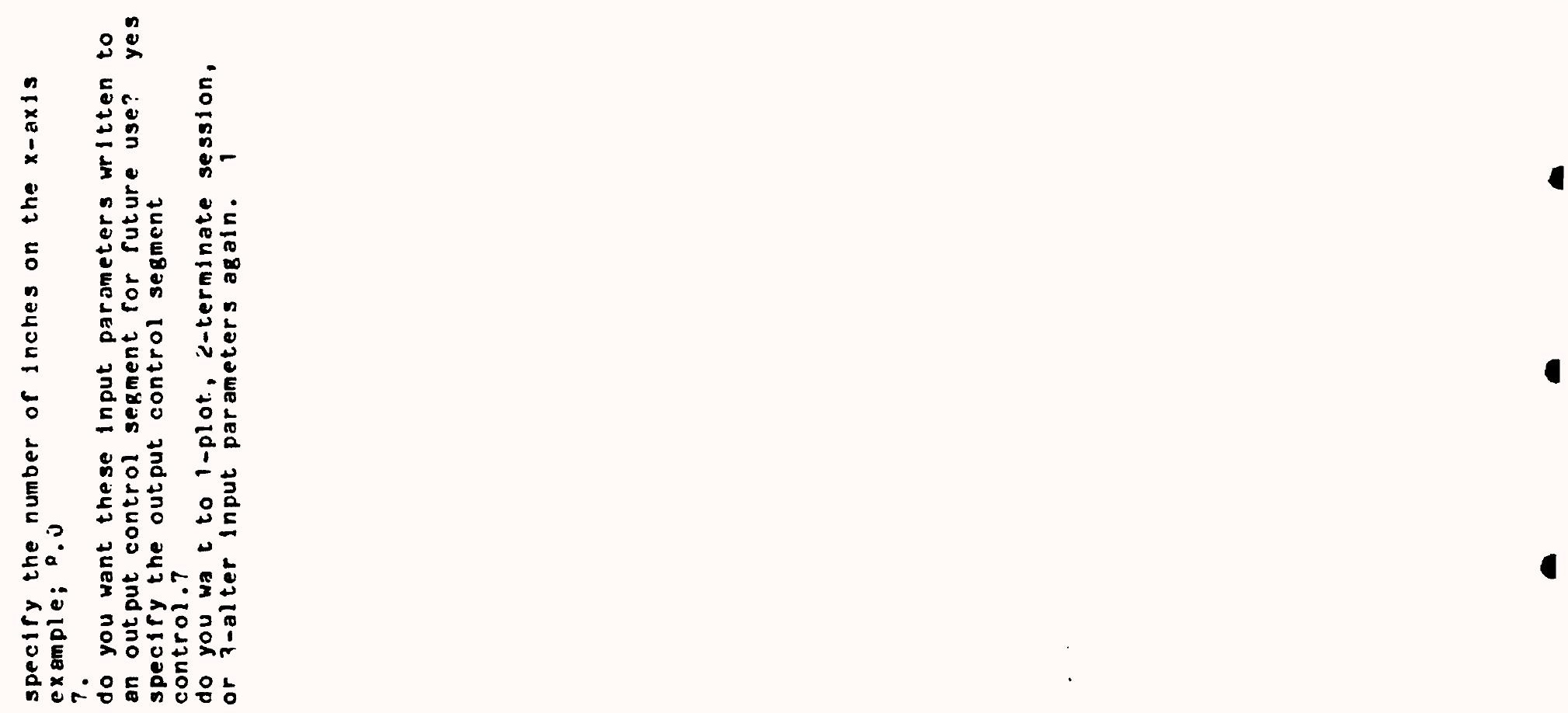
5

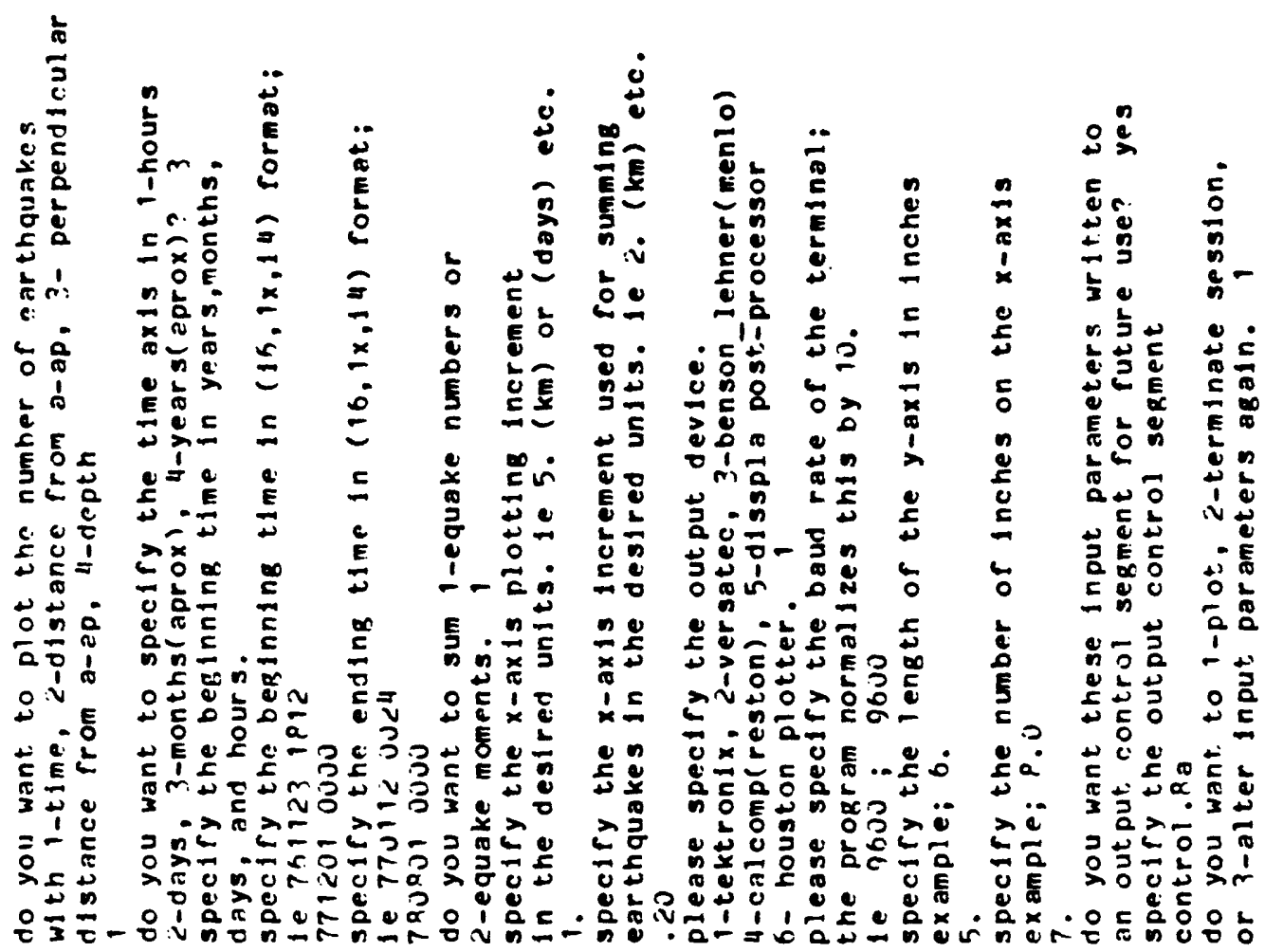

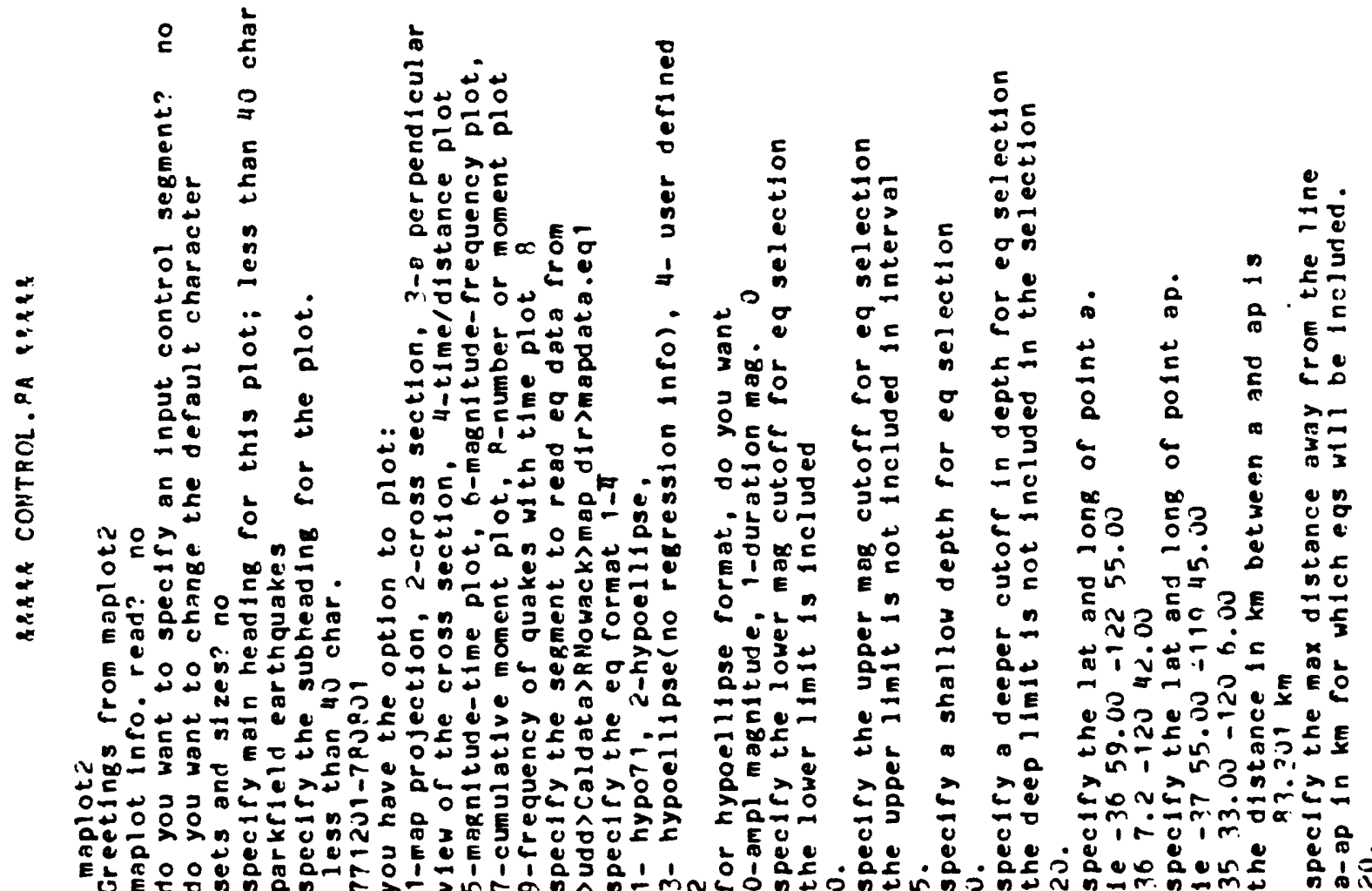



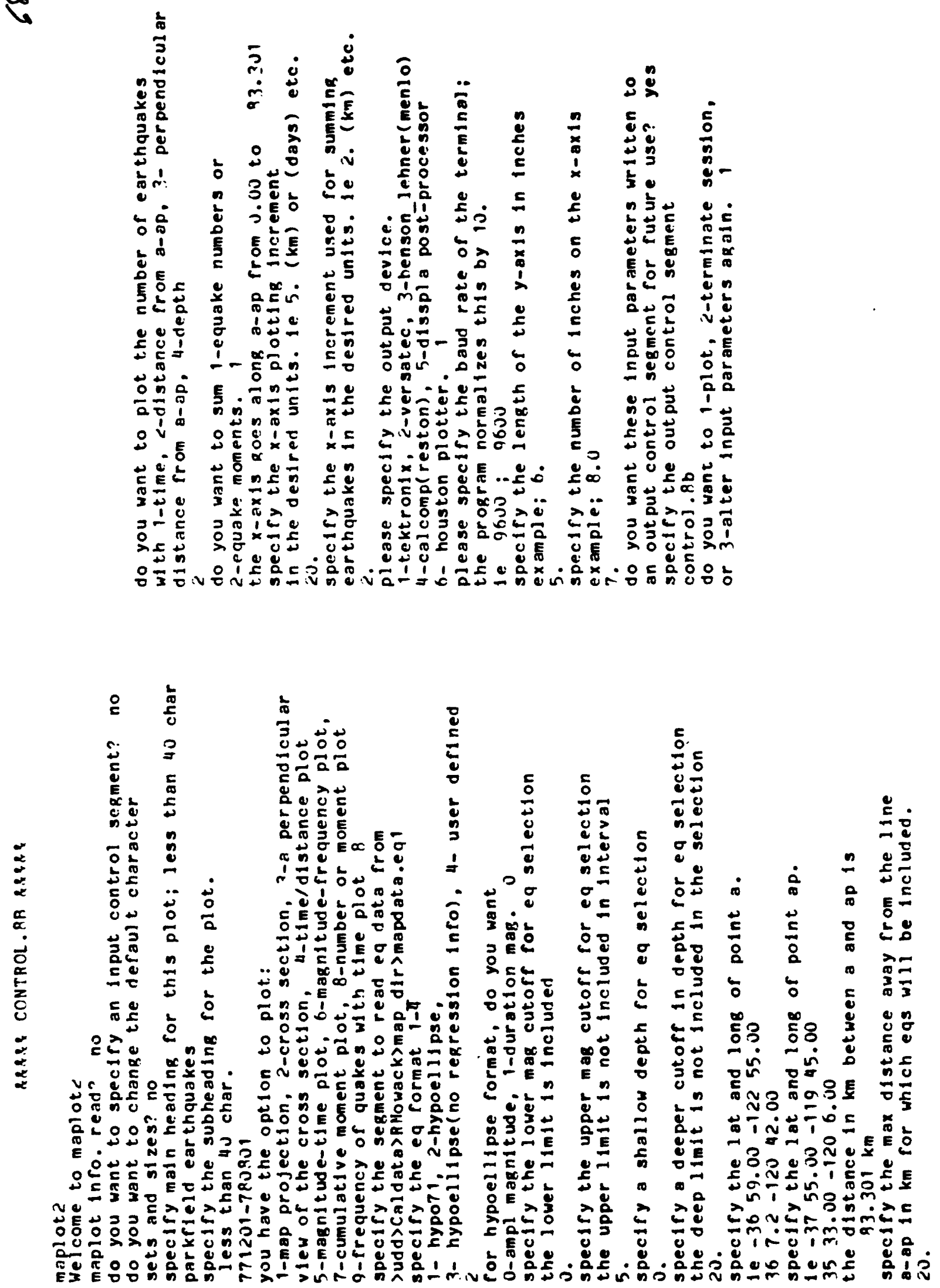

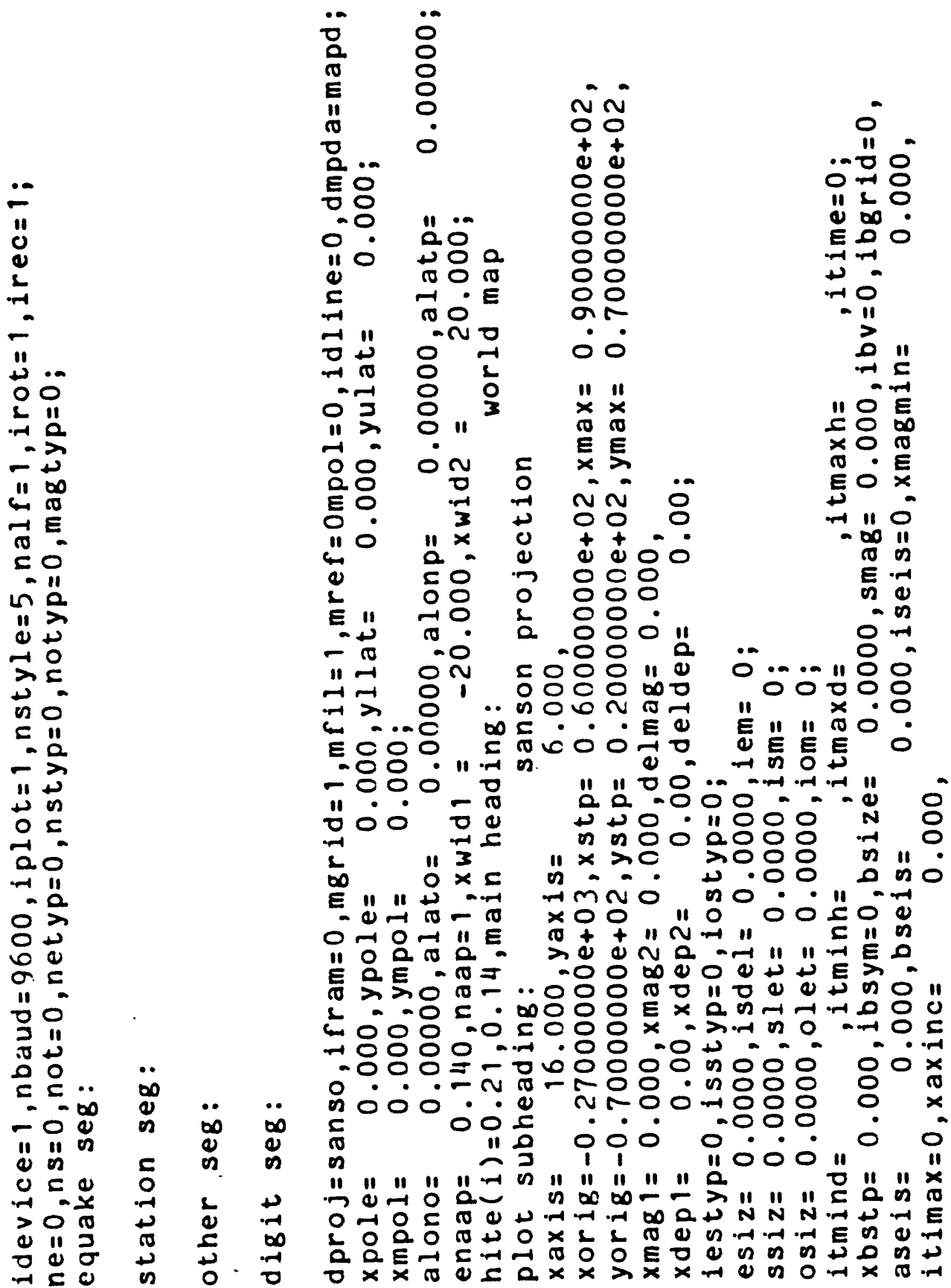


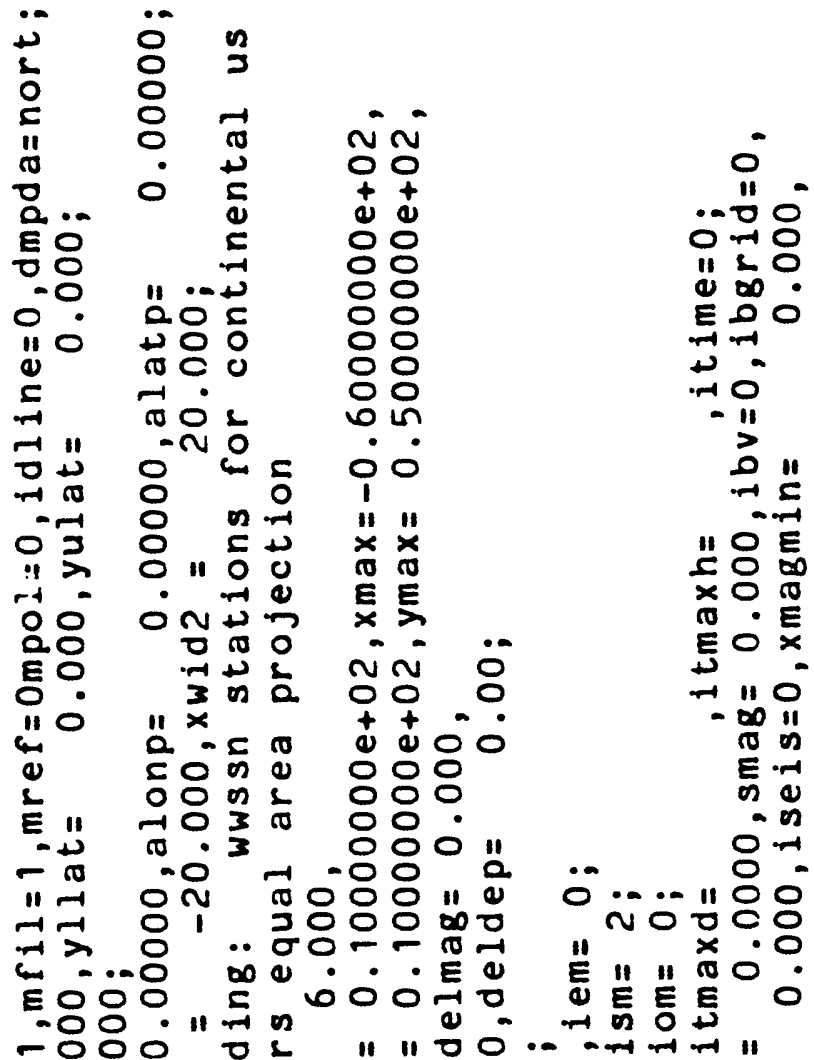

"1

우 워 交 E " $"$ " O

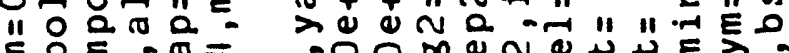
E Q

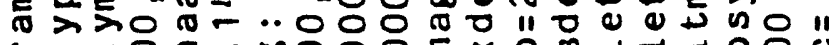

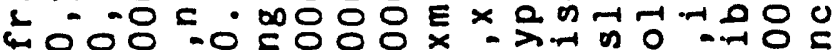
m $0000005000 \times 10$

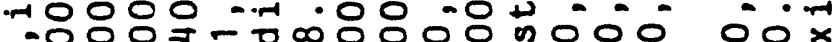

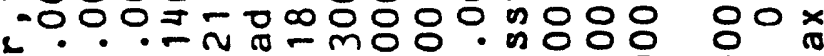
ᄂ00ㅜㄴ

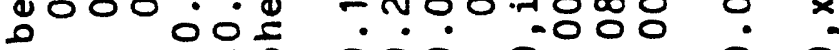
"1 0000000 " " " " " “ ต " "

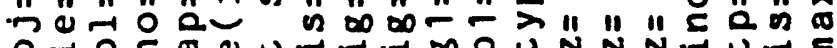

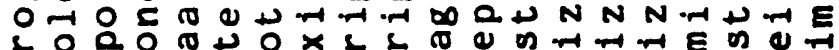

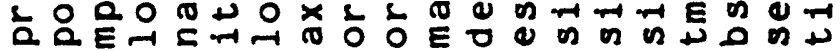

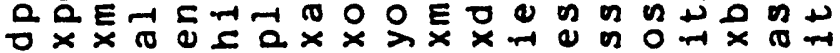




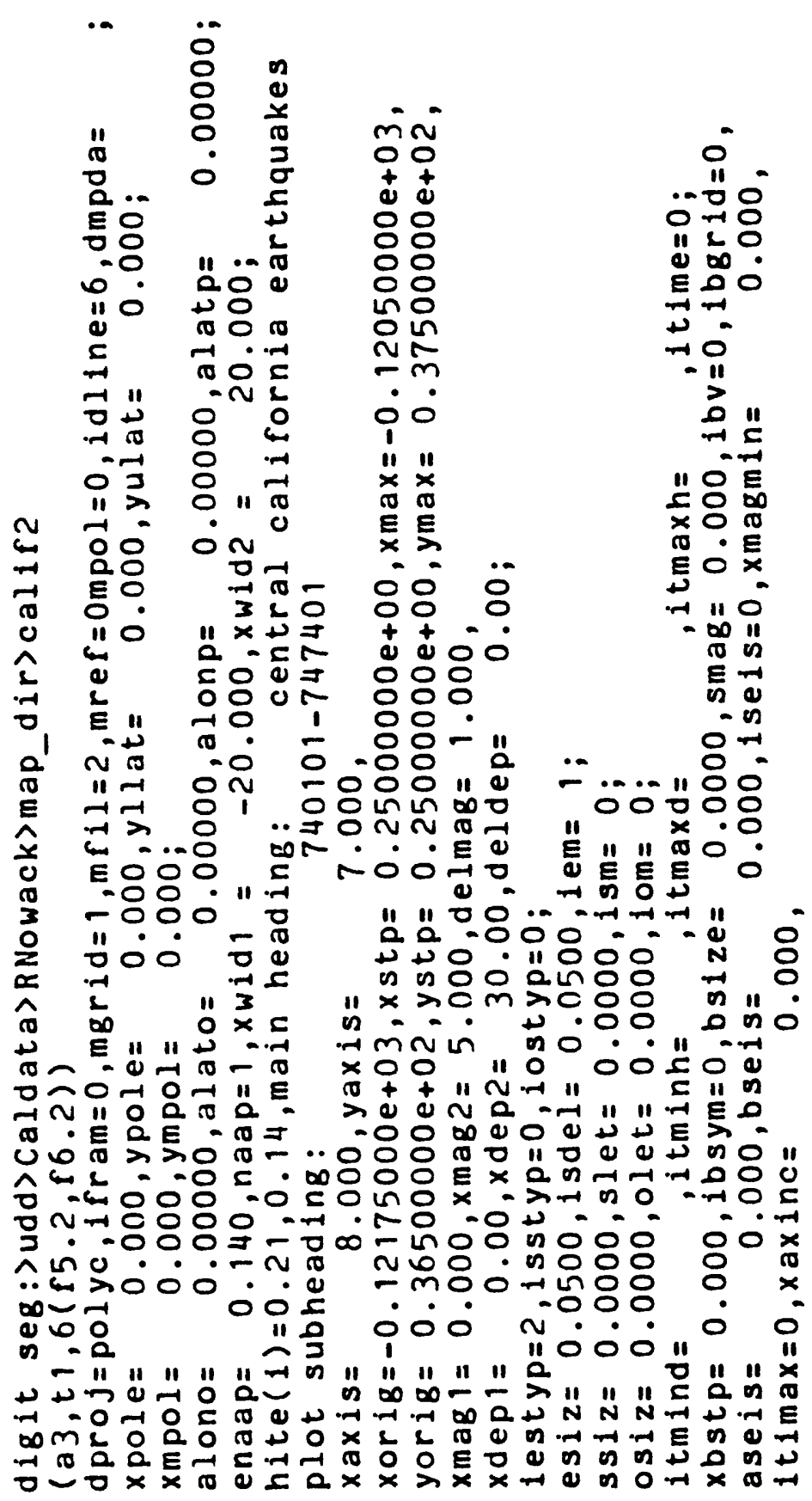




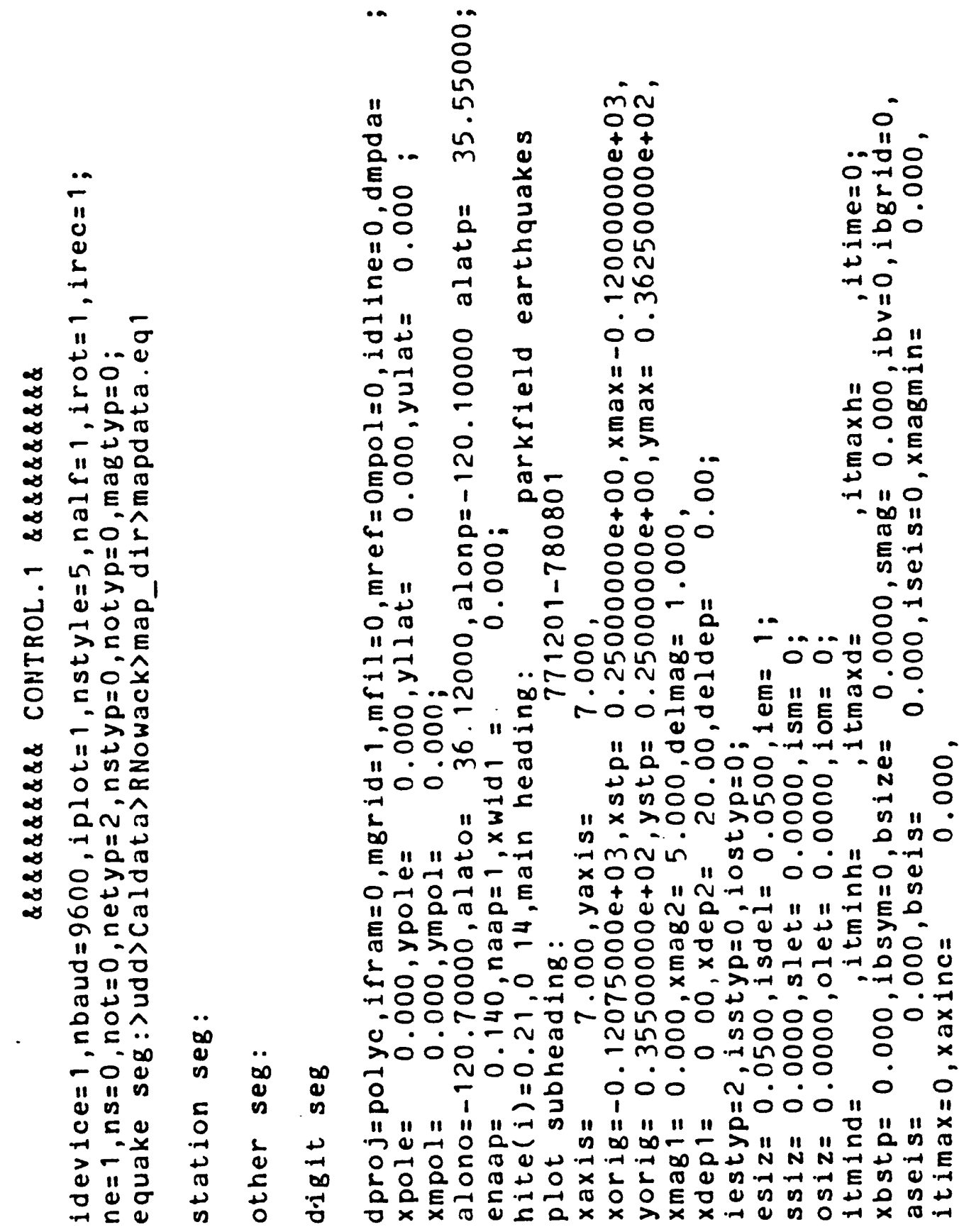




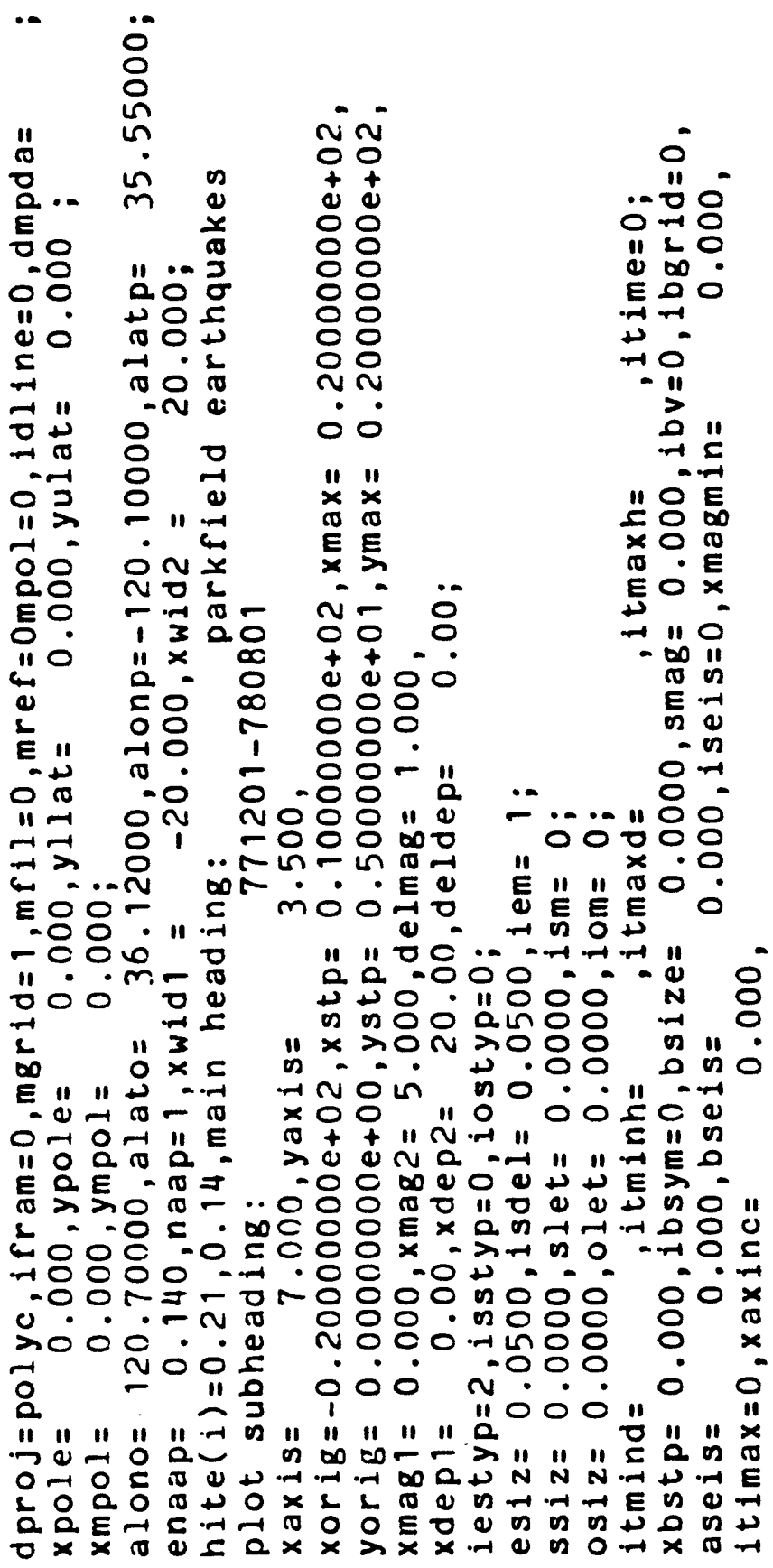




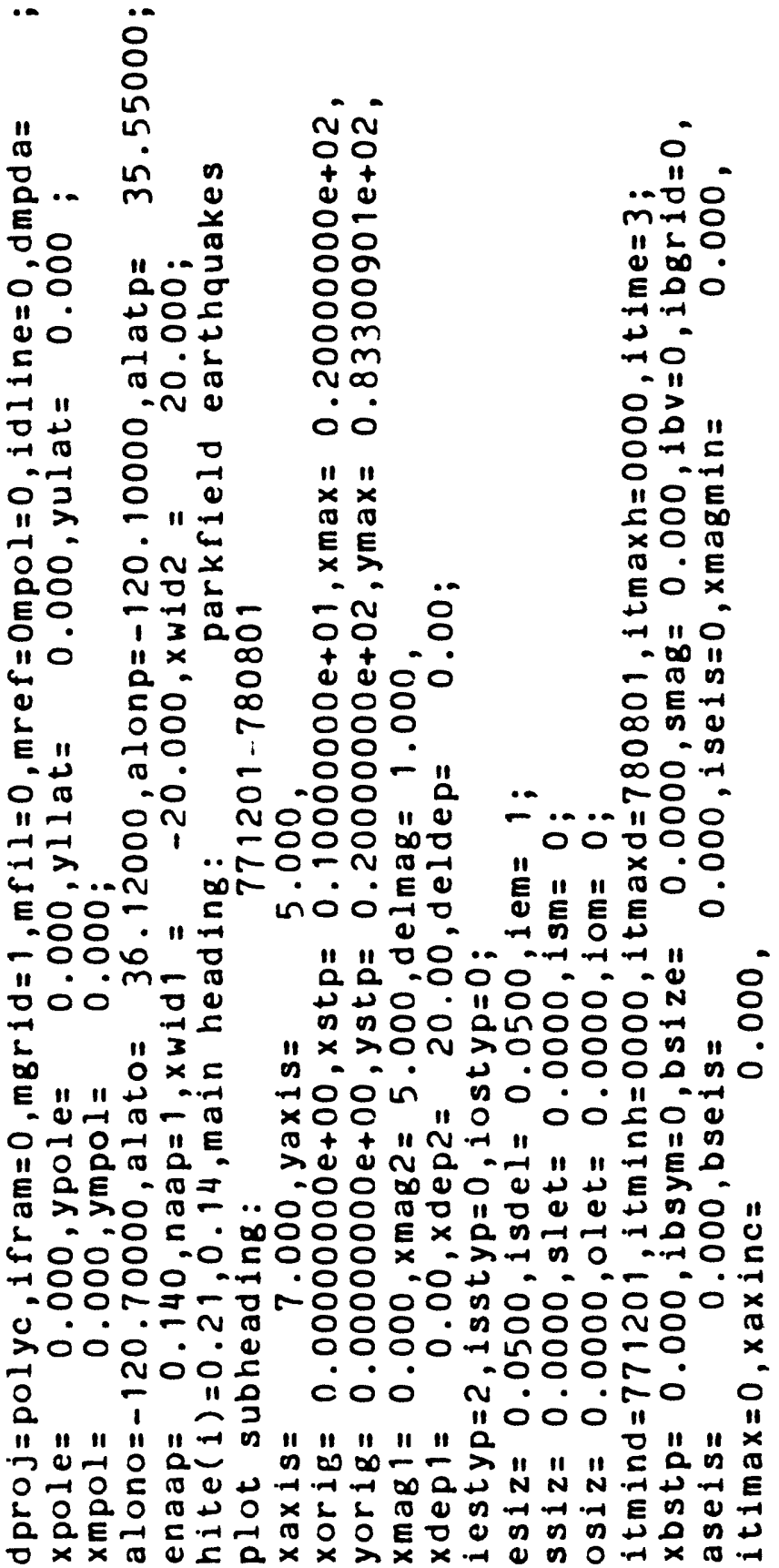


-.

$\ddot{\circ}$
웅
n
n

…

뭉ㅇ

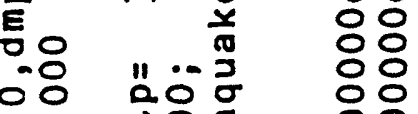

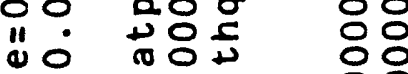

E

$\rightarrow$

D"

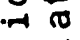

일

$11 \approx$

둥ㅇㅇ

응응

110

4

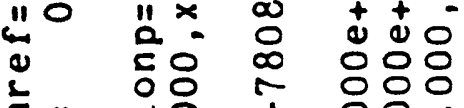

E"

인

$-0$

-

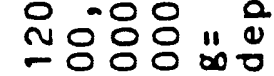

등응

$\rightarrow>$

Eoํำ

nR . . E

-ㅇㅇㅇ.

11

"1 $0 \dot{0} \overline{0}$

$\rightarrow 00 \quad 0$

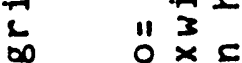

E " 11 د

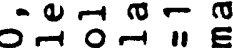

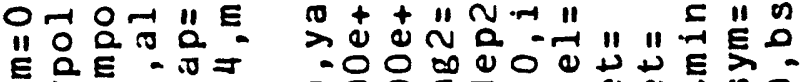

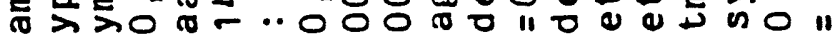

- -

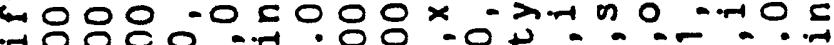
O०ं0.

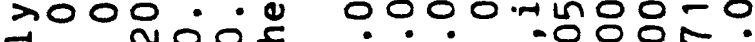

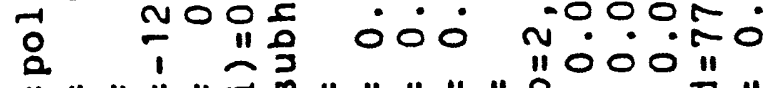
" " a $\rightarrow 0$ a on O- 0 ᄃ

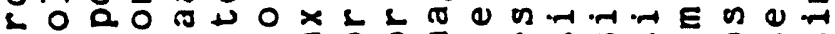
六 员 


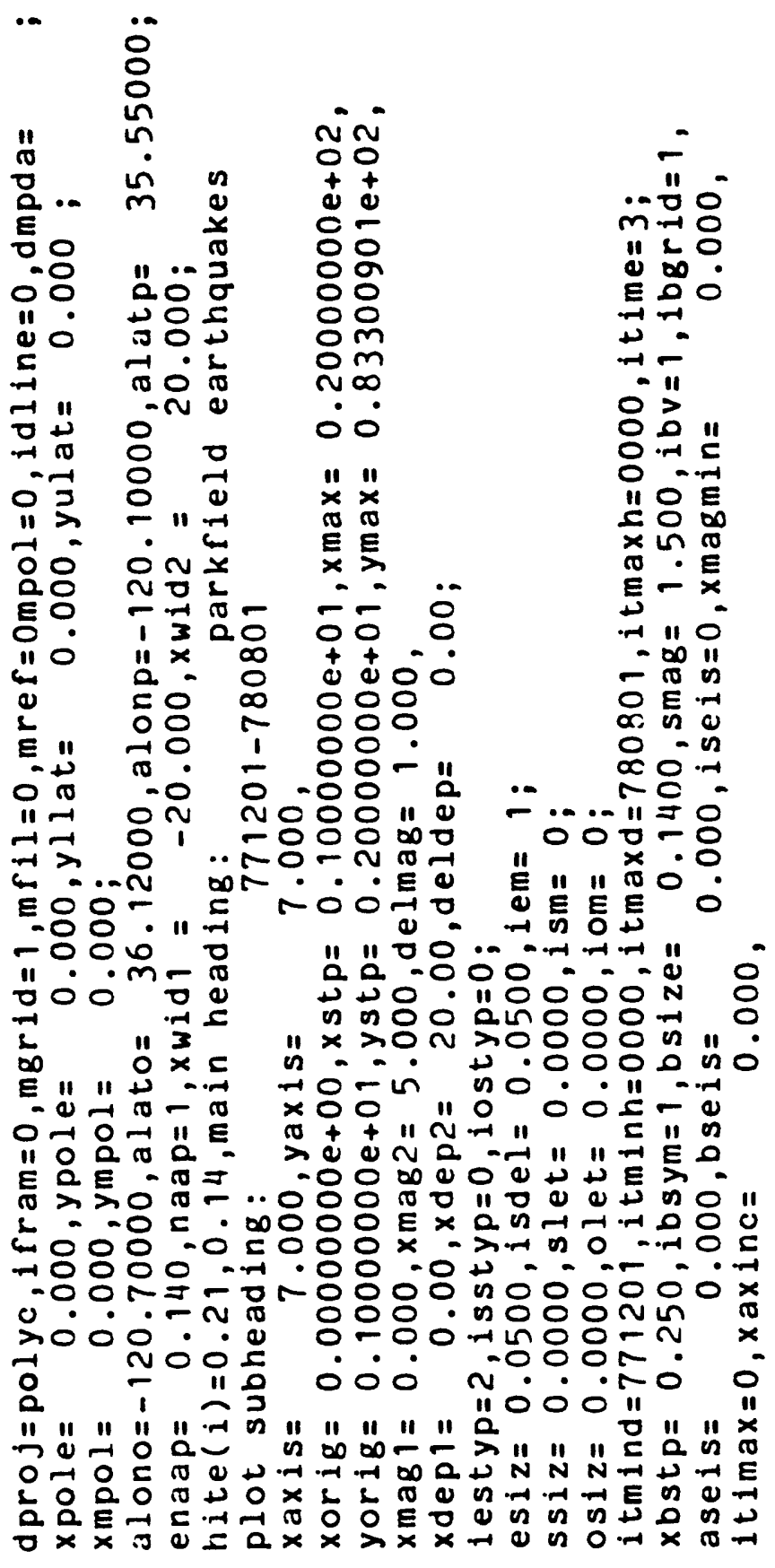




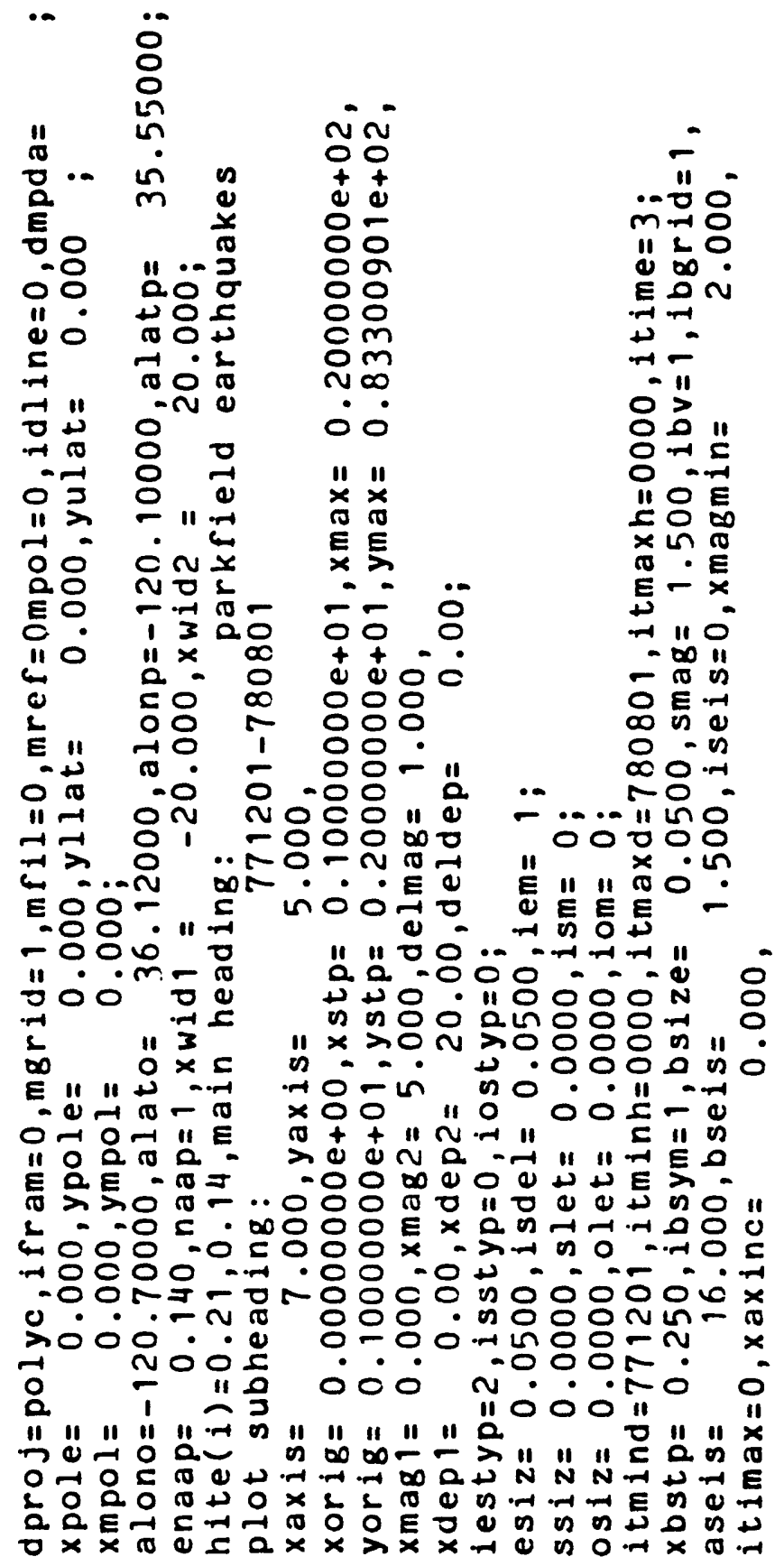




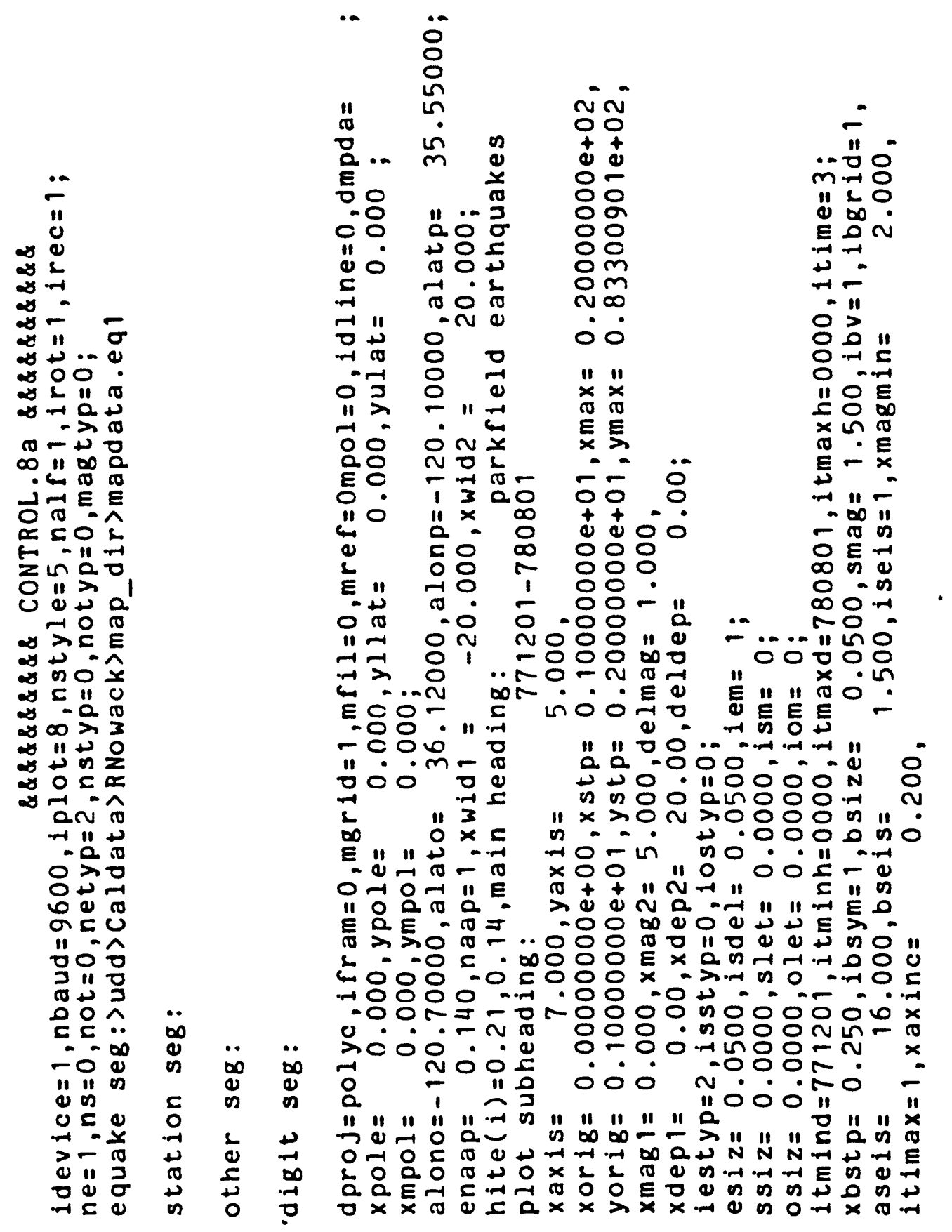




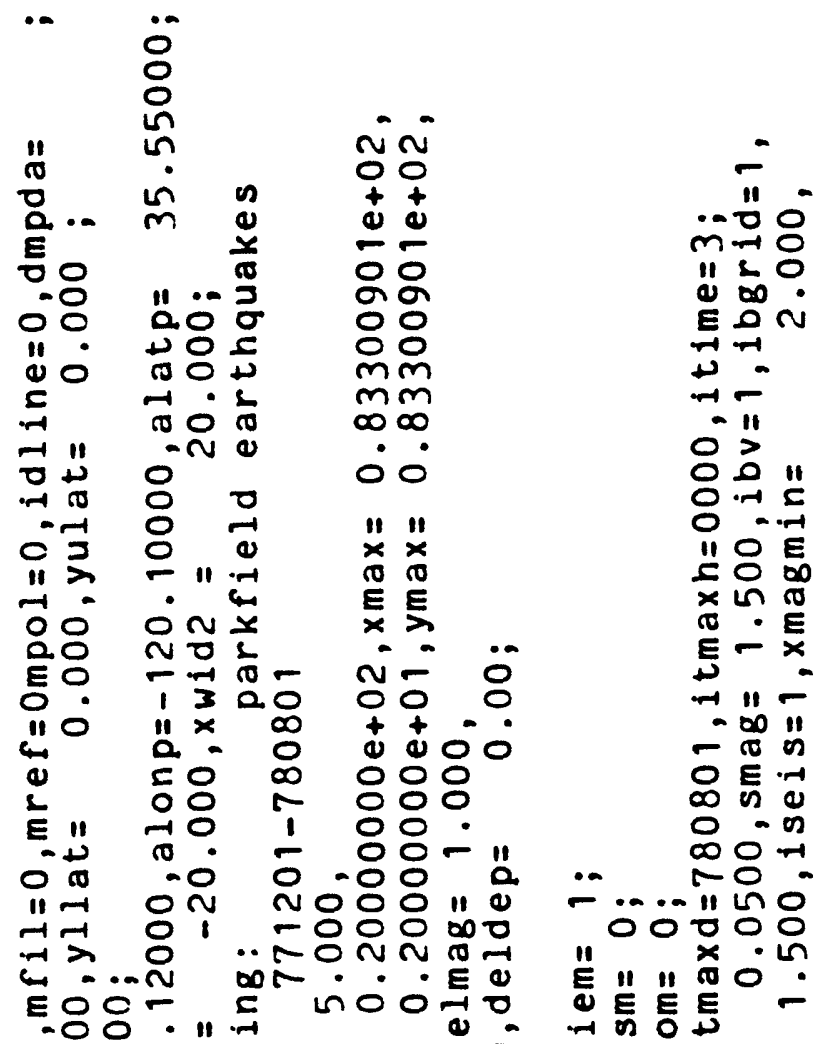

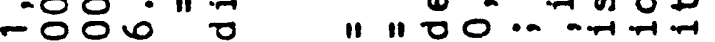
". -m-

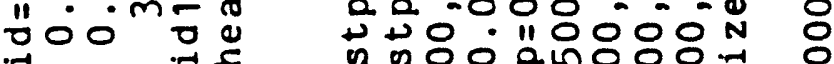
" 1 " E " Oㄱㅁㅔ

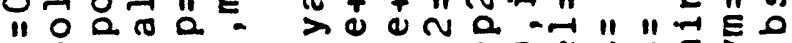

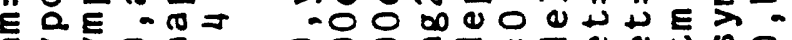

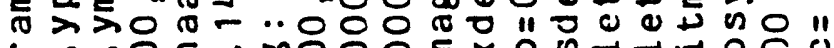
« 4 o. …ㄴ.

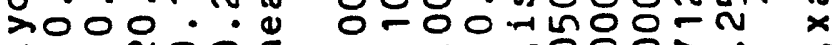
I NoO 믈 "

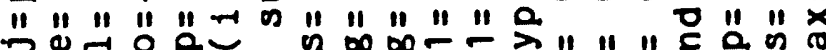

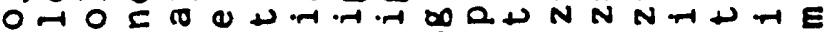

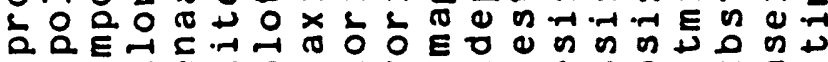

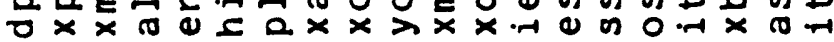


$\bar{u}$

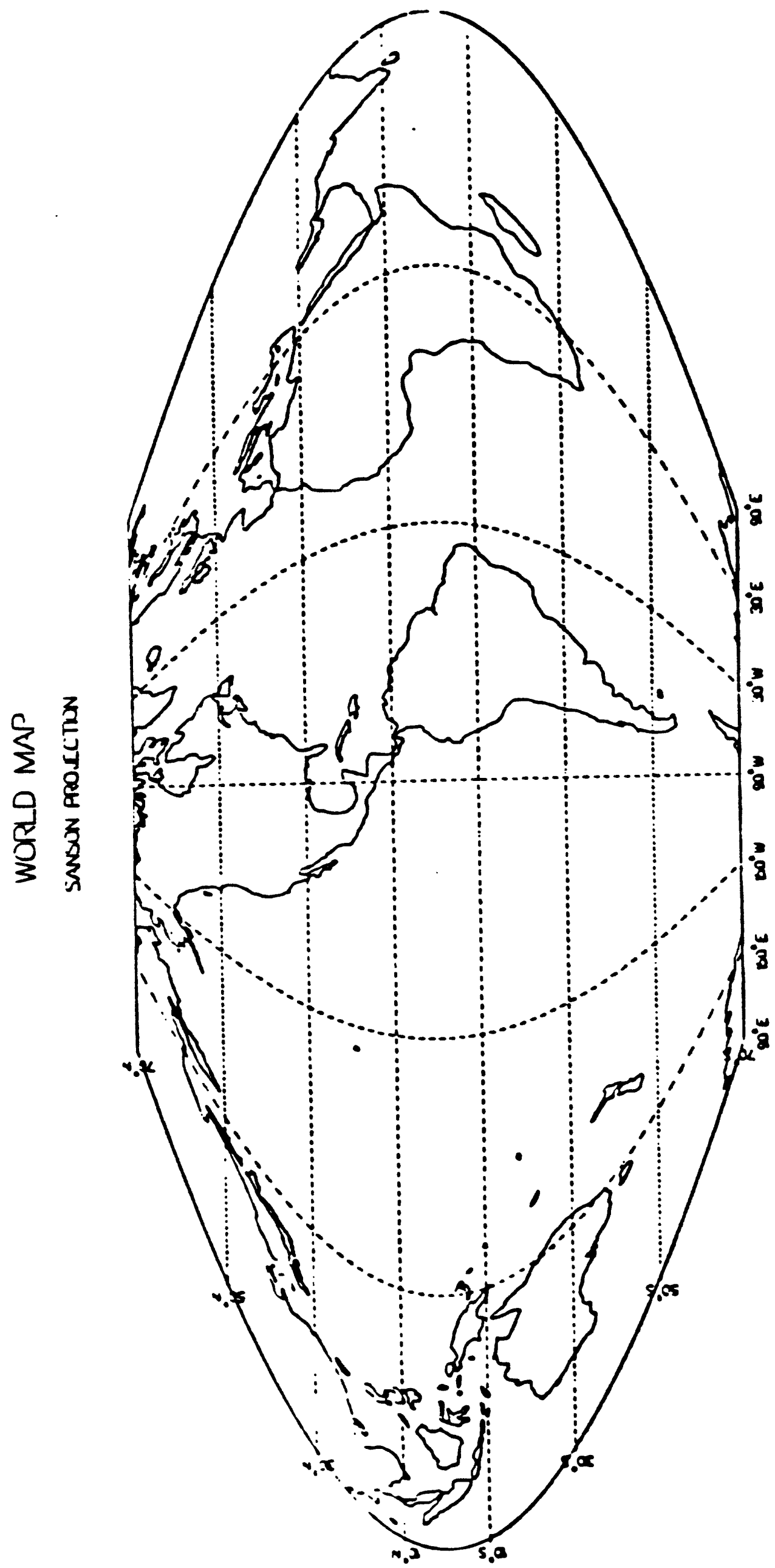




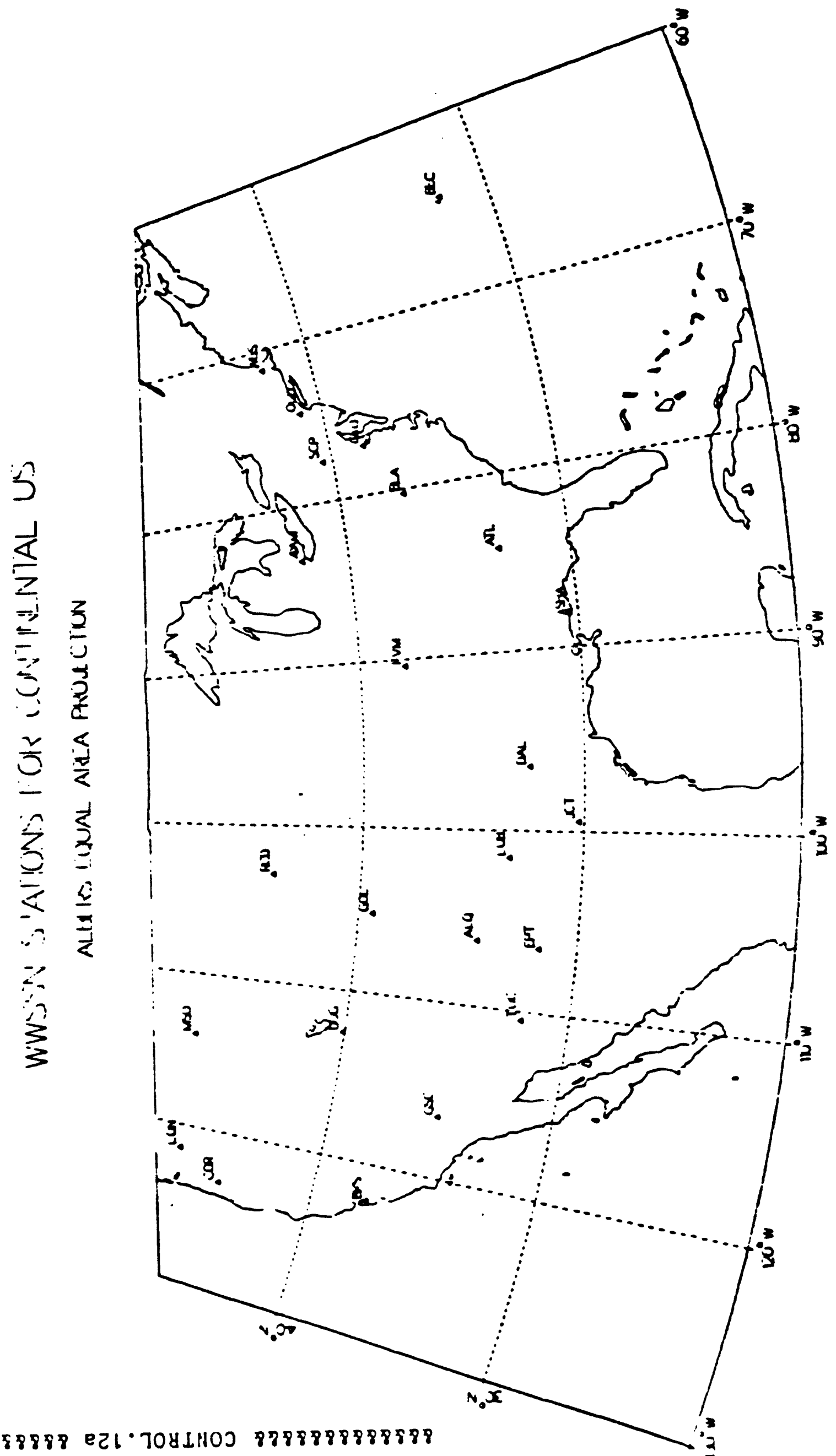




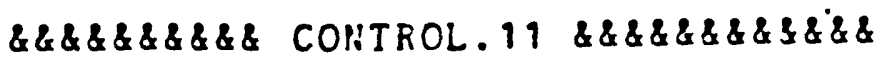

CENTRAL CALFORNIA EARTIRUAIES 74001-74740

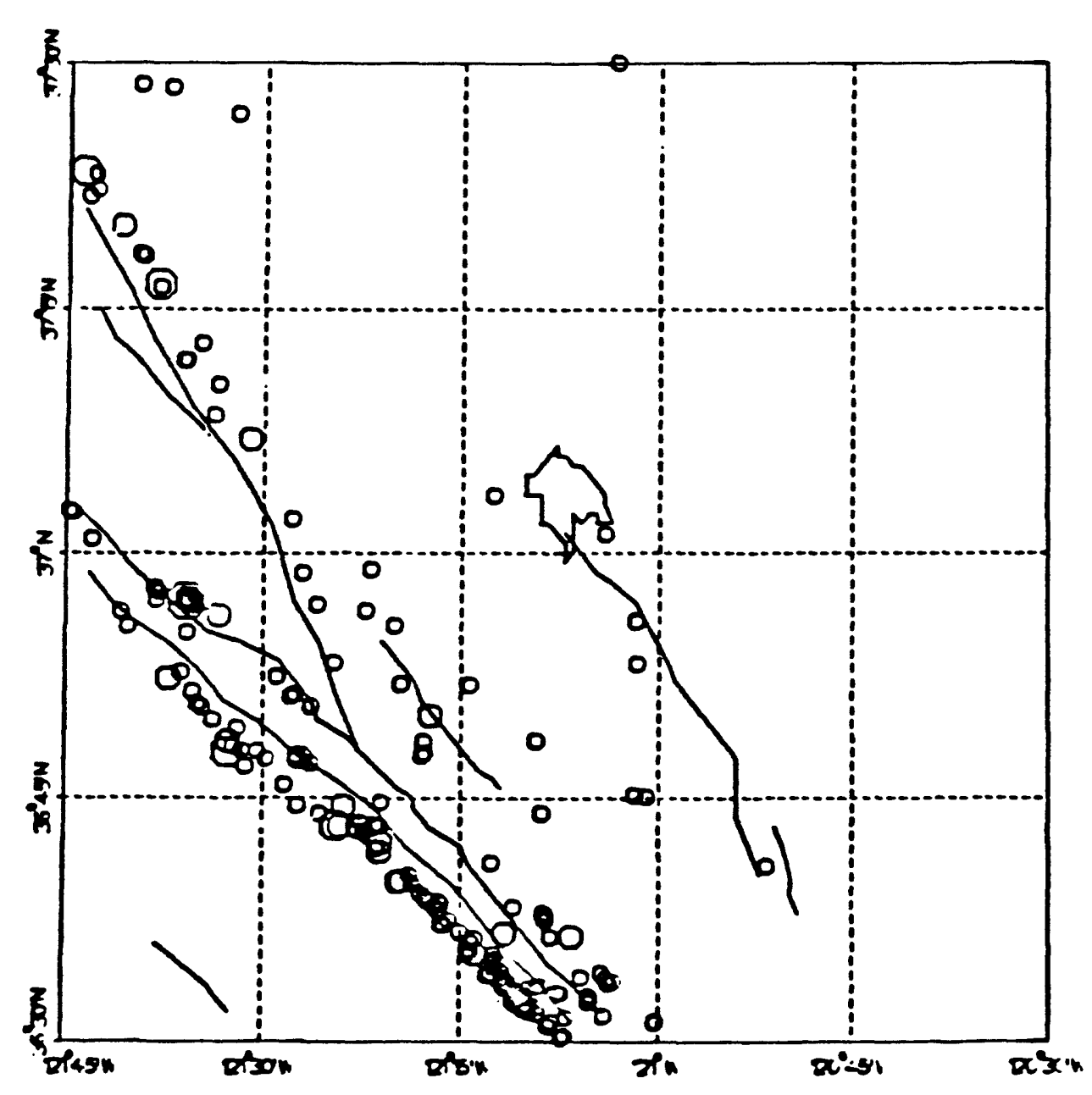


PARKFIELD EARTHQUAKES

771201-780801

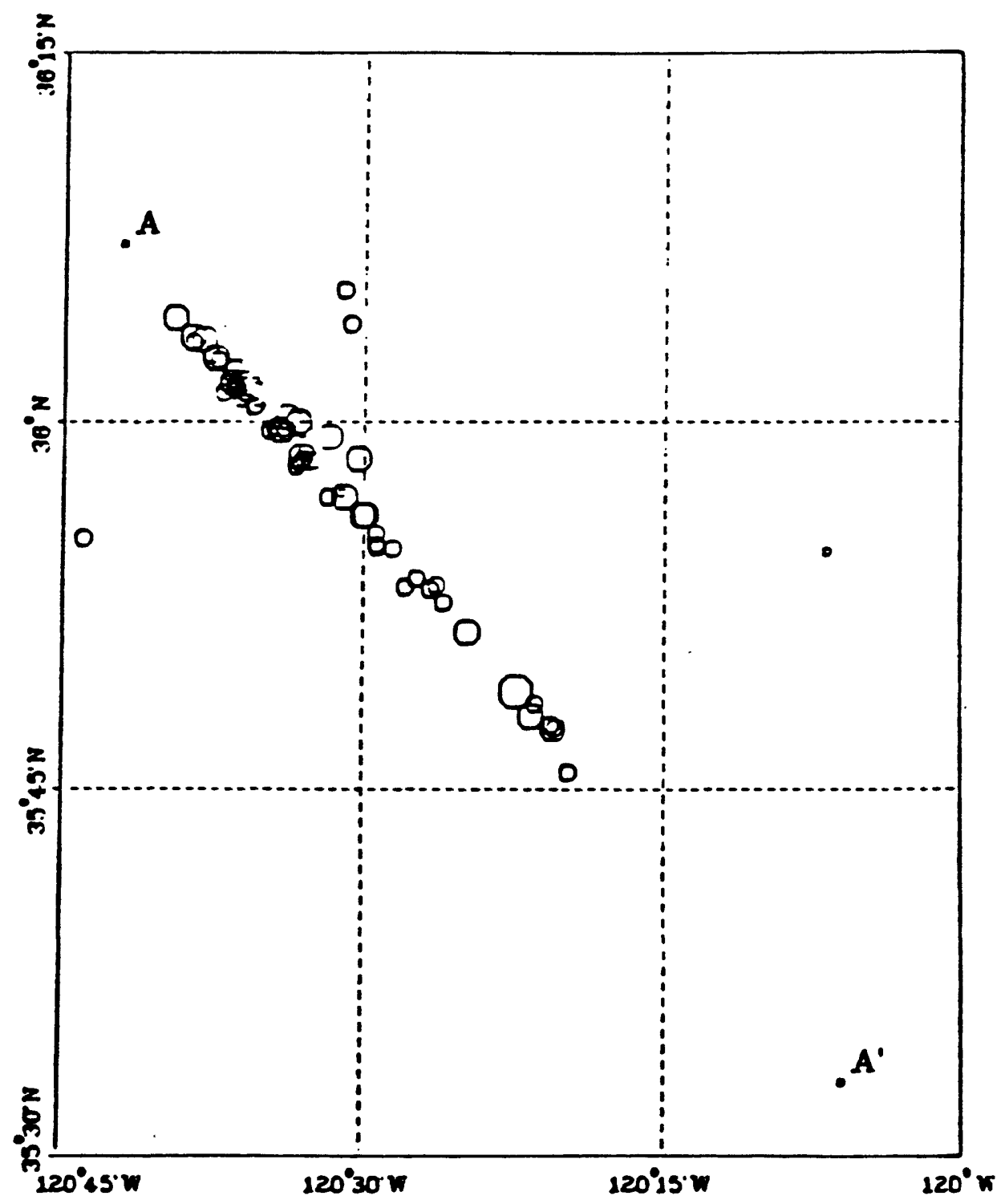




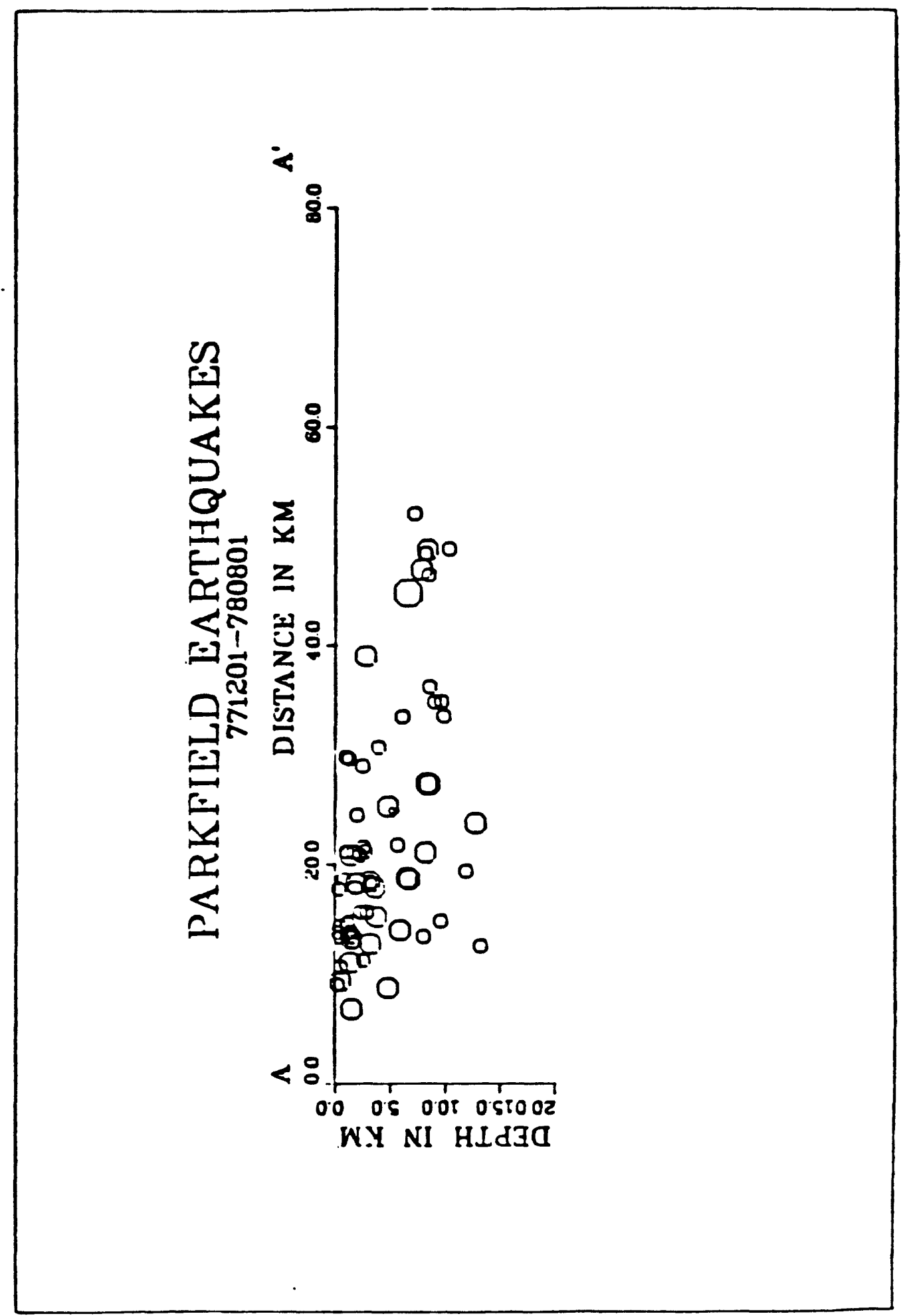




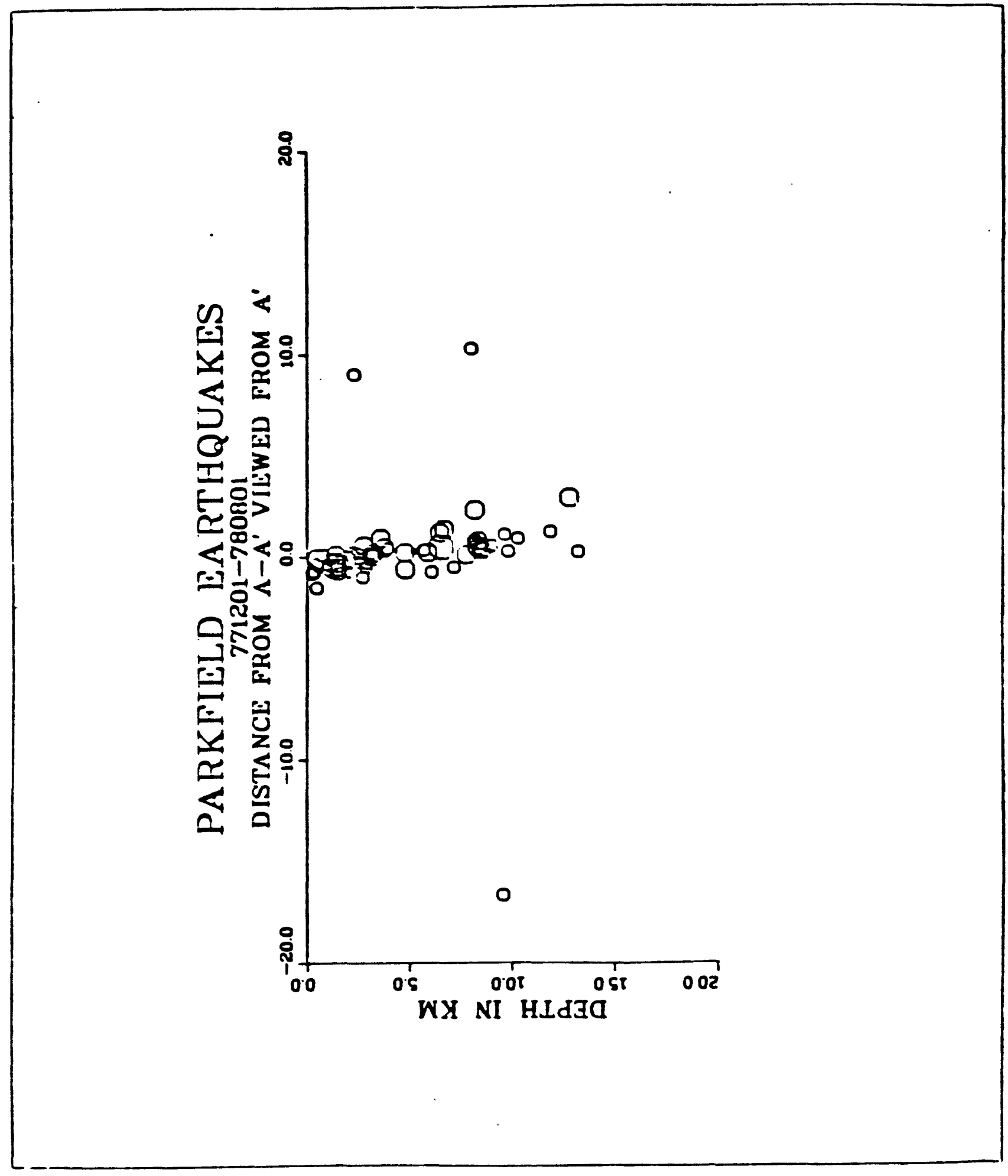




\section{PARKFIELD EARTHQUAKES}

771201-780801

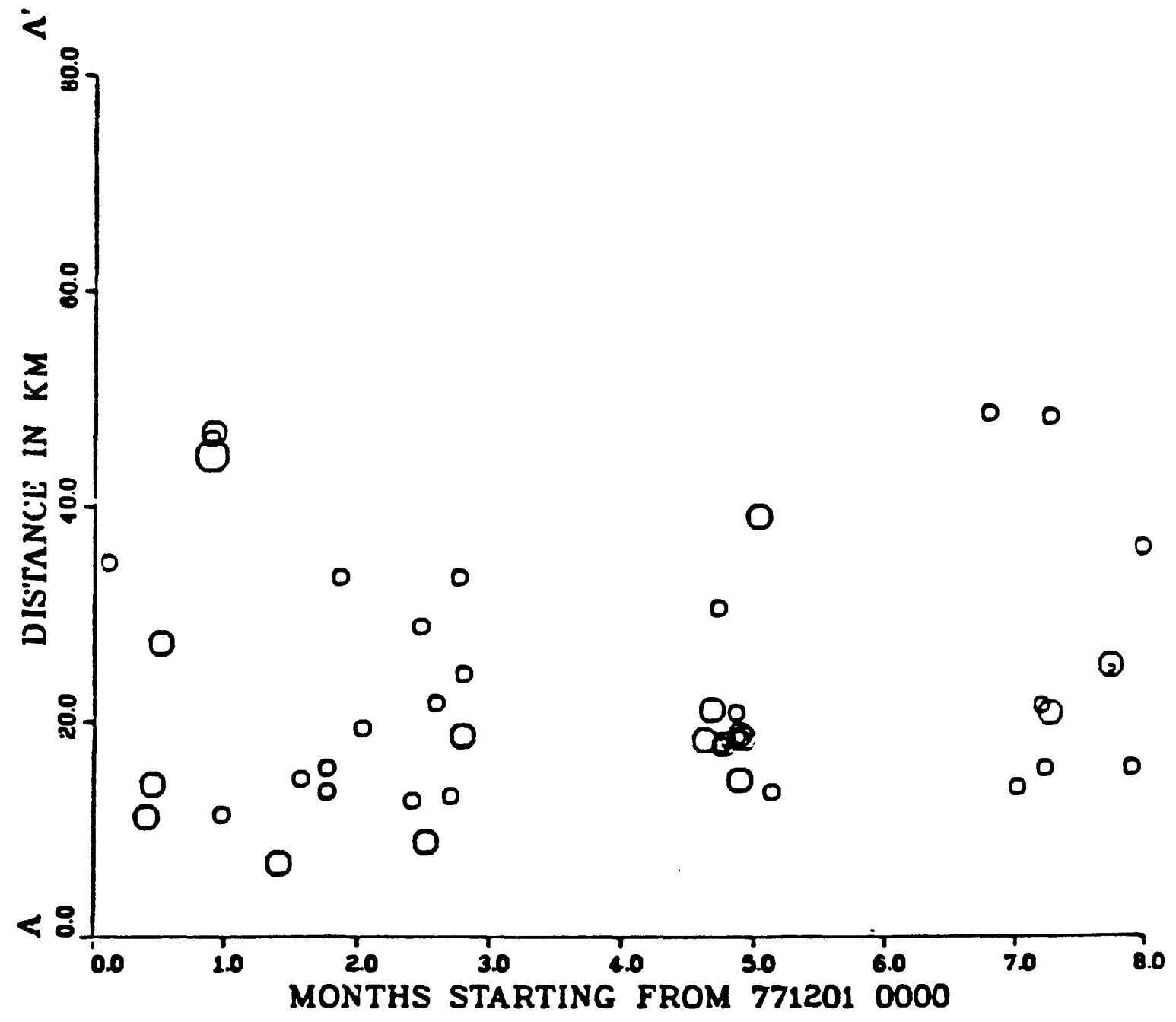




\section{PARKFIELD EARTHQUAKES 771201-780801}

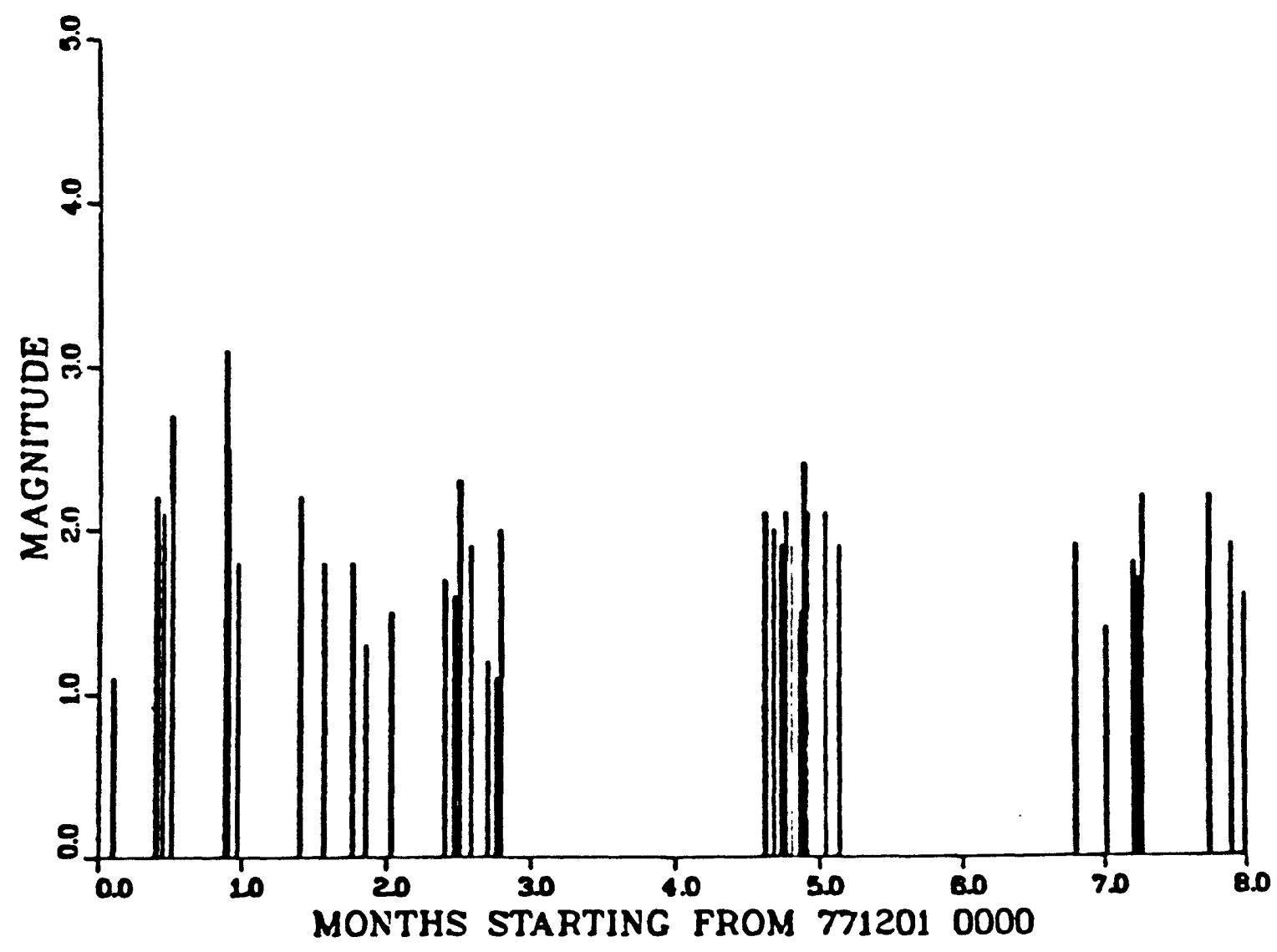




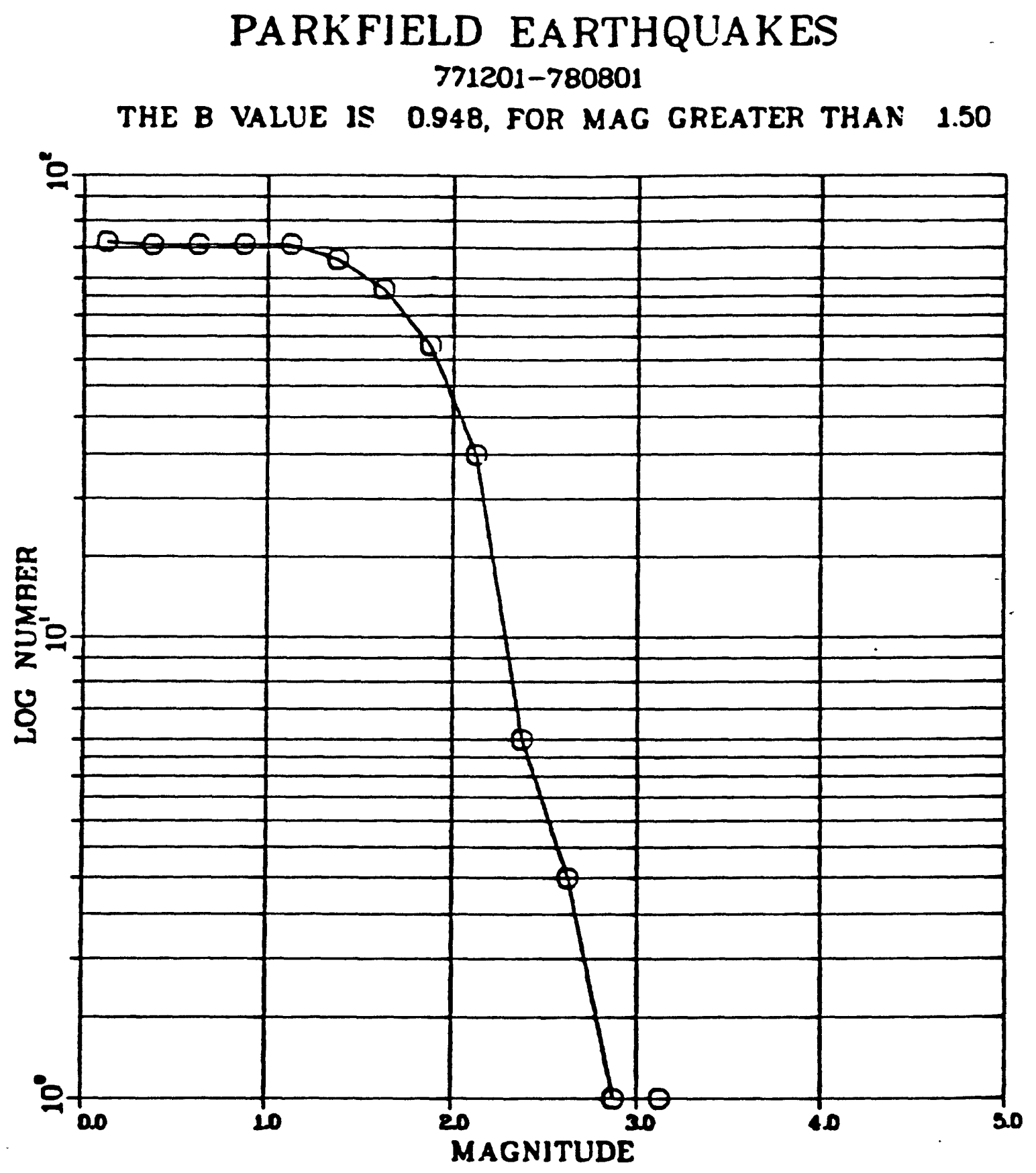




\section{PARKFIELD EARTHQUAKES \\ 771201-780801}

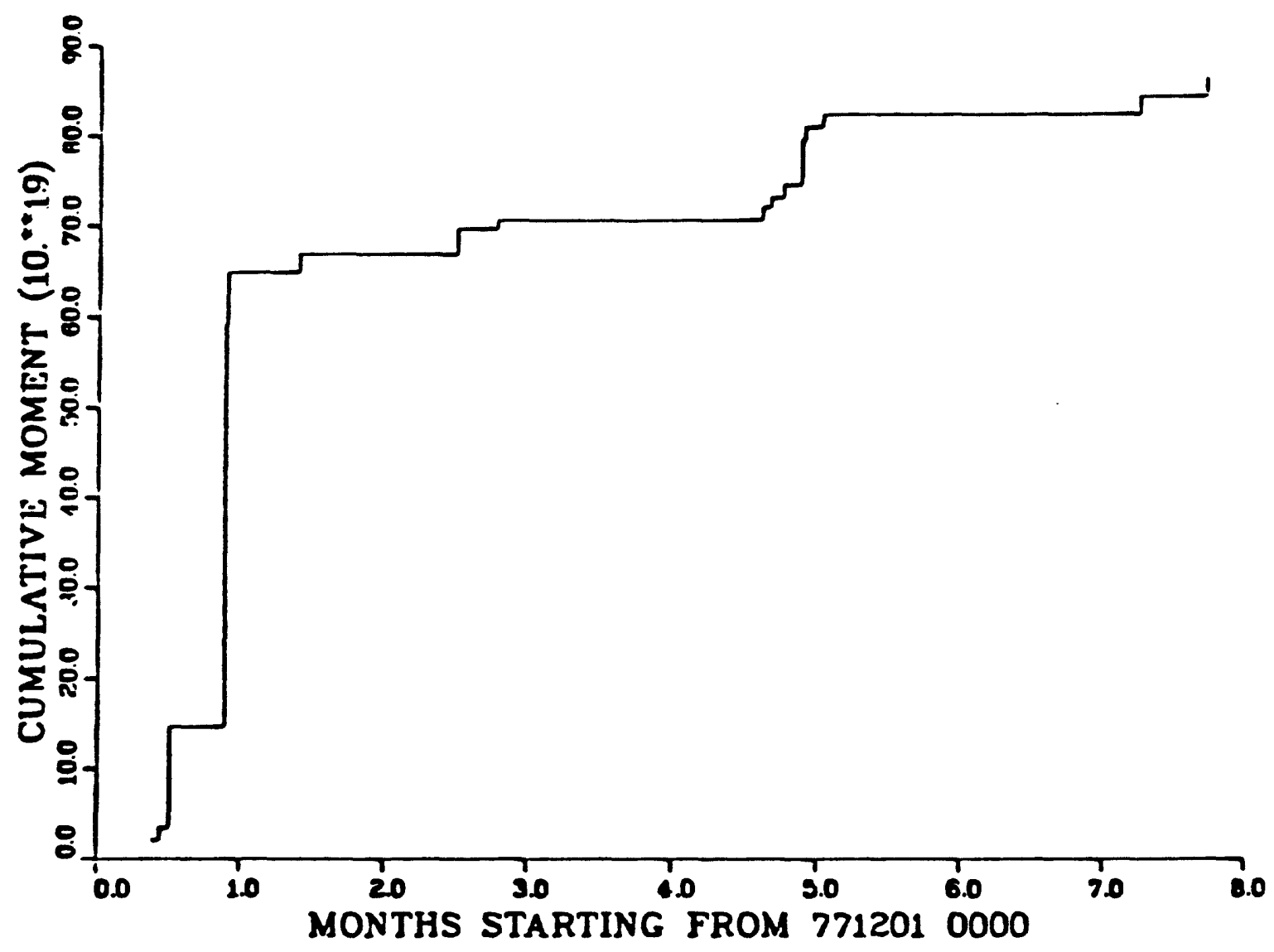




\section{PARKFIELD EARTHQUAKES \\ 771201-780801}

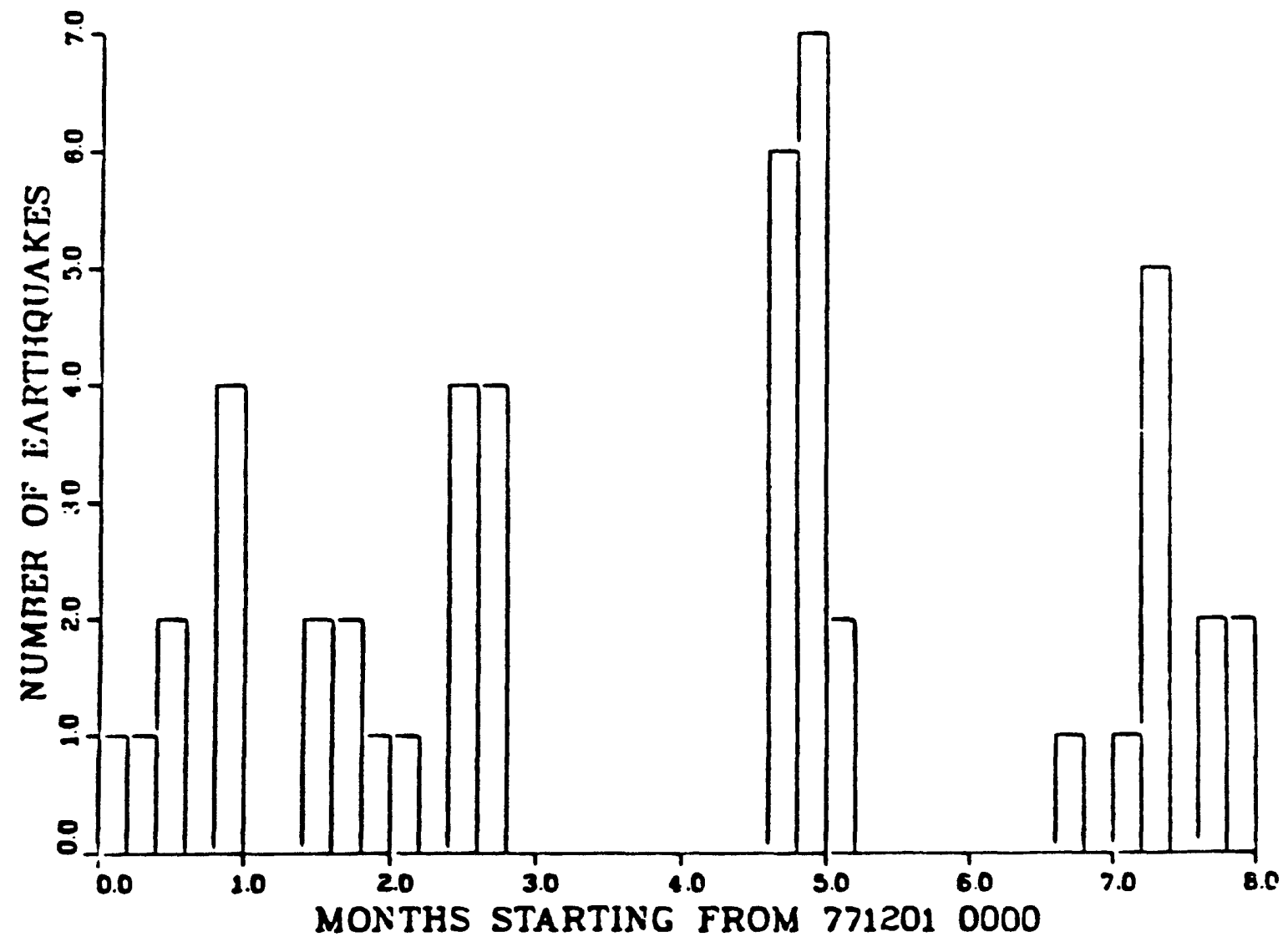




\section{PARKFIELD EARTHQUAKES

$$
\text { 771201-780801 }
$$

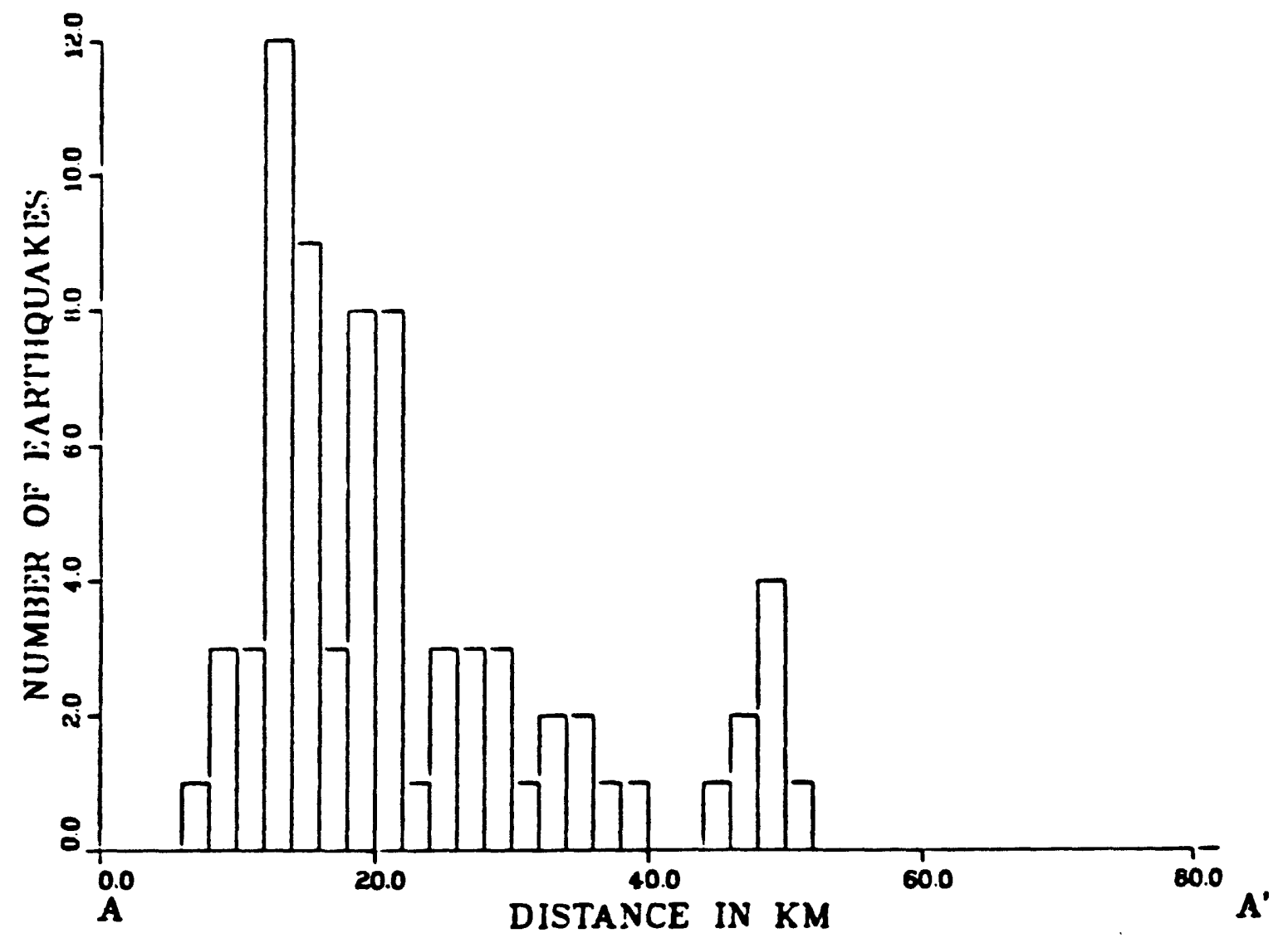

